Prepared in cooperation with the Bureau of Reclamation acting as fiscal agent for the Middle Rio Grande Endangered Species Collaborative Program

\title{
Groundwater Hydrology and Estimation of Horizontal Groundwater Flux from the Rio Grande at Selected Locations in Albuquerque, New Mexico, 2003-9
}

Scientific Investigations Report 2012-5007 



\section{Groundwater Hydrology and Estimation of Horizontal Groundwater Flux from the Rio Grande at Selected Locations in Albuquerque, New Mexico, 2003-9}

By Dale R. Rankin, Kurt J. McCoy, Geoff J.M. Moret, Jeffrey A. Worthington, and Kimberly M. Bandy-Baldwin

Prepared in cooperation with the Bureau of Reclamation acting as fiscal agent for the Middle Rio Grande Endangered Species Collaborative Program

Scientific Investigations Report 2012-5007 


\section{U.S. Department of the Interior \\ KEN SALAZAR, Secretary}

\section{U.S. Geological Survey \\ Suzette M. Kimball, Acting Director}

U.S. Geological Survey, Reston, Virginia: 2013 This and other USGS information products are available at http://store.usgs.gov/
U.S. Geological Survey
Box 25286 , Denver Federal Center
Denver, CO 80225
To learn about the USGS and its information products visit http://www.usgs.gov/
1-888-ASK-USGS

Any use of trade, product, or firm names is for descriptive purposes only and does not imply endorsement by the U.S. Government.

Although this report is in the public domain, permission must be secured from the individual copyright owners to reproduce any copyrighted materials contained within this report.

Suggested citation:

Rankin, D.R., McCoy, K.J., Moret, G.J.M., Worthington, J.S., and Bandy-Baldwin, K.M., 2013, Groundwater hydrology and estimation of horizontal groundwater flux from the Rio Grande at selected locations in Albuquerque, New Mexico, 2003-9: U.S. Geological Survey Scientific Investigations Report 2012-5007, 75 p. 


\section{Acknowledgments}

The authors would like to recognize the efforts of others who contributed to the completion of this study. The City of Albuquerque's Open Space Division granted permission for the USGS to install and maintain piezometers in the Rio Grande riparian zone along the river. The Middle Rio Grande Conservancy District granted permission for the USGS to install and maintain surfacewater gages at various locations in the Corrales, Atrisco, and Albuquerque Riverside Drains. The Bernalillo County Commission granted permission for the USGS to install and maintain piezometers at various locations near the $\mathrm{I}-25$ bridge and the Barelas bridge.

The authors wish to recognize the support of committees associated with the Middle Rio Grande Endangered Species Collaborative Program, including the Executive Committee, the Science Subcommittee, and, in particular, members of the Species and Water Management Committee (SWM). We wish to acknowledge the assistance and guidance provided by Charles Fischer and Valda Terauds of the Bureau of Reclamation. In addition, we would like to thank the U.S. Army Corps of Engineers for the initial funding of the monitoring network, the installation of which preceded the interpretive work done in cooperation with the Bureau of Reclamation Endangered Species Collaborative Program.

We would also like to acknowledge the technical assistance provided by Jeb Brown, a hydrologic technician in the New Mexico Water Science Center, who performed the discharge measurements for the seepage investigation. 


\section{Contents}

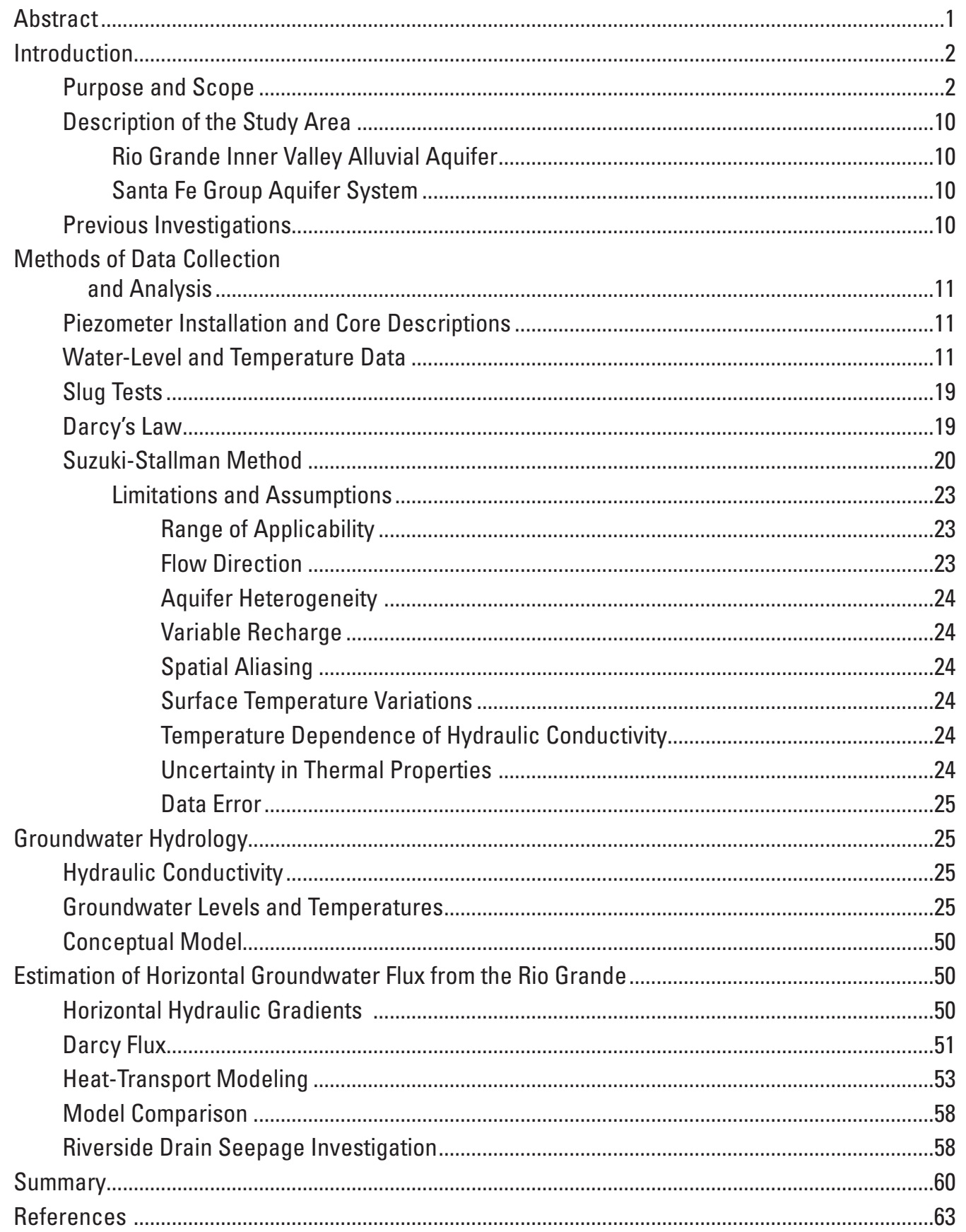




\section{Figures}

1. Map showing location of study area and transects in the Albuquerque area, New Mexico

2. Map showing groundwater-level-elevation contours in the Santa Fe Group aquifer in the Albuquerque area and estimated groundwater-level declines from 1960 to 2002.

3a. Map showing location of piezometer nests and surface-water gages and water-table contours showing direction of groundwater flow at the

Paseo del Norte transects, November 13, 2008...

4. Diagram showing of sets of piezometers used to compute daily mean hydraulic gradients on each side of the Rio Grande for each paired transect

5. Graph showing type curves for the Suzuki-Stallman method, showing values of the parameters $a$ and $b$ as a function of specific flux.

6. Boxplot showing summary of hydraulic conductivities estimated from slug tests conducted at selected locations in the Rio Grande inner valley alluvial aquifer, Albuquerque, New Mexico...

7a. Hydrographs showing daily mean stage of the Rio Grande and riverside drains and daily mean groundwater levels in piezometers at Paseo Del Norte transect 1

8a. Graph showing daily mean water temperature in the Rio Grande and riverside drains and daily mean groundwater temperature in piezometers at Paseo del Norte transect 1

9. Graph showing vertical temperature profiles in piezometers, winter nonirrigation season, October 2008, January 2009, and February 2009

10. Graph showing conceptual model of groundwater flow from the Rio Grande through the Rio Grande inner valley alluvial aquifer

11. Graph showing daily mean Rio Grande stage and daily mean Darcy fluxes calculated from hydraulic gradients and hydraulic conductivities from slug tests, and from hydraulic gradients and a range of hydraulic conductivities

12. Boxplot showing summary of mean annual specific flux through the Rio Grande inner valley alluvial aquifer, Albuquerque, New Mexico, calculated using Suzuki-Stallman method 


\section{Tables}

1. Site data for piezometers and surface-water data collection sites, Rio Grande inner valley, Albuquerque, New Mexico.

2. Summary of Bouwer and Rice (1976) and Butler (1998) slug-test results from piezometers in the Rio Grande inner valley alluvial aquifer, Albuquerque, New Mexico

3. Median annual magnitude of groundwater horizontal hydraulic gradient and direction of groundwater flow at piezometer transects, Rio Grande inner valley alluvial aquifer, Albuquerque, New Mexico, 2006-9

4. Suzuki-Stallman results for horizontal flux between temperature time-series pairs in the Rio Grande inner valley alluvial aquifer, Albuquerque, New Mexico, 2006-8

5. Comparison of Darcy's law and Suzuki-Stallman calculations of horizontal groundwater flux in the Rio Grande inner valley alluvial aquifer, Albuquerque, New Mexico.

6. Seepage investigation discharge measurements in the Corrales and Albuquerque Riverside drains, Albuquerque, New Mexico, February 26, 2009 


\section{Conversion Factors and Datums}

\begin{tabular}{|c|c|c|}
\hline Multiply & & To obtain \\
\hline \multicolumn{3}{|c|}{ Length } \\
\hline inch (in.) & 2.54 & centimeter $(\mathrm{cm})$ \\
\hline inch (in.) & 25.4 & millimeter (mm) \\
\hline foot $(\mathrm{ft})$ & 0.3048 & meter $(\mathrm{m})$ \\
\hline mile (mi) & 1.609 & kilometer $(\mathrm{km})$ \\
\hline \multicolumn{3}{|c|}{ Area } \\
\hline acre & 0.004047 & square kilometer $\left(\mathrm{km}^{2}\right)$ \\
\hline square mile $\left(\mathrm{mi}^{2}\right)$ & 2.590 & square kilometer $\left(\mathrm{km}^{2}\right)$ \\
\hline \multicolumn{3}{|c|}{ Volume } \\
\hline gallon (gal) & 3.785 & liter $(\mathrm{L})$ \\
\hline gallon (gal) & 0.003785 & cubic meter $\left(\mathrm{m}^{3}\right)$ \\
\hline cubic foot $\left(\mathrm{ft}^{3}\right)$ & 0.02832 & cubic meter $\left(\mathrm{m}^{3}\right)$ \\
\hline \multicolumn{3}{|c|}{ Flow rate } \\
\hline foot per day (ft/d) & 0.3048 & meter per day $(\mathrm{m} / \mathrm{d})$ \\
\hline foot per second (ft/s) & 0.3048 & meter per second $(\mathrm{m} / \mathrm{s})$ \\
\hline cubic foot per second $\left(\mathrm{ft}^{3} / \mathrm{s}\right)$ & 0.02832 & cubic meter per second $\left(\mathrm{m}^{3} / \mathrm{s}\right)$ \\
\hline cubic foot per day $\left(\mathrm{ft}^{3} / \mathrm{d}\right)$ & 0.02832 & cubic meter per day $\left(\mathrm{m}^{3} / \mathrm{d}\right)$ \\
\hline gallon per minute (gal/min) & 0.06309 & liter per second $(\mathrm{L} / \mathrm{s})$ \\
\hline gallons per day (gal/d) & 0.003785 & cubic meter per day $\left(\mathrm{m}^{3} / \mathrm{d}\right)$ \\
\hline inch per year (in/yr) & 25.4 & millimeter per year (mm/yr) \\
\hline \multicolumn{3}{|c|}{ Temperature } \\
\hline degree Fahrenheit $\left({ }^{\circ} \mathrm{F}\right)$ & ${ }^{\circ} \mathrm{F}-32 / 1.8$ & degree Celsius $\left({ }^{\circ} \mathrm{C}\right)$ \\
\hline \multicolumn{3}{|c|}{ Thermal Conductivity } \\
\hline $\begin{array}{l}\text { British thermal unit }{ }_{\mathrm{IT}} \text { per hour foot } \\
\text { degree Fahrenheit }\left(\mathrm{BTU}_{\mathrm{IT}} \mathrm{hr}^{-1}\right. \\
\left.\mathrm{ft}^{-1}{ }^{\circ} \mathrm{F}^{-1}\right)\end{array}$ & 1.730 & watt per meter Kelvin $\left(\mathrm{W} \mathrm{m}^{-1} \mathrm{~K}^{-1}\right)$ \\
\hline \multicolumn{3}{|c|}{ Volumetric Heat Capacity } \\
\hline $\begin{array}{l}\text { British Thermal Unit (International } \\
\text { Steam Table Calorie) per cubic } \\
\text { foot degree Fahrenheit (BTU }{ }_{\text {IT }} \\
\mathrm{ft}^{-3}{ }^{\circ} \mathrm{F}^{-1} \text { ) }\end{array}$ & 67070 & joule per cubic meter Kelvin $\left(\mathrm{J} \mathrm{m}^{-3} \mathrm{~K}^{-1}\right)$ \\
\hline
\end{tabular}





\title{
Groundwater Hydrology and Estimation of Horizontal Groundwater Flux from the Rio Grande at Selected Locations in Albuquerque, New Mexico, 2003-9
}

\author{
By Dale R. Rankin, ${ }^{1}$ Kurt J. McCoy, ${ }^{2}$ Geoff J.M. Moret, ${ }^{3}$ Jeffrey A. Worthington, ${ }^{1}$ and \\ Kimberly M. Bandy-Baldwin ${ }^{4}$
}

\section{Abstract}

The Albuquerque, New Mexico, area has two principal sources of water: groundwater from the Santa Fe Group aquifer system and surface water from the San JuanChama Diversion Project. From 1960 to 2002, groundwater withdrawals from the Santa Fe Group aquifer system have caused water levels to decline more than 120 feet in some places within the Albuquerque area, resulting in a great deal of interest in quantifying the river-aquifer interaction associated with the Rio Grande.

In 2003, the U.S. Geological Survey in cooperation with the Bureau of Reclamation, the Middle Rio Grande Endangered Species Collaborative Program, and the U.S. Army Corps of Engineers began a detailed characterization of the hydrogeology of the Rio Grande riparian corridor in the Albuquerque, New Mexico, area to provide hydrologic data and enhance the understanding of rates of water leakage from the Rio Grande to the alluvial aquifer, groundwater flow through the aquifer, and discharge of water from the aquifer to the riverside drains.

A simple conceptual model of flow indicates that the groundwater table gently slopes from the Rio Grande towards riverside drains and the outer boundaries of the inner valley. Water infiltrating from the Rio Grande initially moves vertically below the river, but, as flow spreads farther into the Rio Grande inner valley alluvial aquifer, flow becomes primarily horizontal. The slope of the water-table surface may

${ }^{1}$ U.S. Geological Survey, New Mexico Water Science Center, 5338 Montgomery Blvd. NE, Albuquerque, NM, 87059

${ }^{2}$ U.S. Geological Survey, Virginia Water Science Center, 1730 East Parham Road, Richmond, VA, 23228

${ }^{3}$ University of Idaho, Department of Fish and Wildlife Resources, PO Box 441136, Moscow, ID, 83844

${ }^{4}$ Colorado School of Mines, Department of Geology and Geological Engineering, 1516 Illinois St., Golden, CO, 80401 be strongly controlled by the riverside drains and influenced by other more distal hydrologic boundary conditions, such as groundwater withdrawals by wells.

Results from 35 slug tests performed in the Rio Grande inner valley alluvial aquifer during January and February 2009 indicate that hydraulic-conductivity values ranged from 5 feet per day to 160 feet per day with a median hydraulicconductivity for all transects of 40 feet per day. Median annual horizontal hydraulic gradients in the Rio Grande inner valley alluvial aquifer ranged from 0.011 to 0.002 .

Groundwater fluxes through the alluvial aquifer calculated by using median slug-test results $\left(q_{\text {slug }}\right)$ and Darcy's law ranged from about 0.1 feet per day to about 0.7 feet per day. Groundwater fluxes calculated by using the SuzukiStallman method $\left(q_{\text {heat }}\right)$ ranged from 0.52 feet per day to 0.23 feet per day.

Results from the Darcy's law and Suzuki-Stallman flux calculations were compared to discharge measured in riverside drains on both sides of the river north of the Montaño Bridge on February 26, 2009. Flow in the Corrales Riverside Drain increased by 1.4 cubic feet per second from mile 2 to mile 4 , about 12 cubic feet per day per linear foot of drain. Flow in the Albuquerque Riverside Drain increased by 15 cubic feet per second between drain miles 0 and 3, about 82 cubic feet per day per linear foot of drain.

The flux of water from the river to the aquifer was calculated to be 2.2 cubic feet per day per linear foot of river by using the median $q_{\text {slug }}$ of 0.09 feet per day at Montaño transects west of the river. The total flux was calculated to be 6.0 cubic feet per day per linear foot of river by using the mean $q_{\text {heat }}$ of 0.24 feet per day for the Montaño transects west of the river. Assuming the Corrales Riverside Drain intercepted all of this flow, the $q_{\text {slug }}$ or $q_{\text {heat }}$ fluxes account for 18 to 50 percent, respectively, of the increase of flow in the drain. The flux of water from the river to the aquifer was calculated to be 15 cubic feet per day per linear foot of river by using the median $q_{\text {slug }}$ of 0.30 feet per day at the Montaño transects east of the river. The flux of water from the river to the aquifer was calculated to be 17 cubic feet per day per linear foot of river by using the mean flux calculated from the 
Suzuki-Stallman method for the Montaño East transects of 0.34 feet per day. Assuming the Albuquerque Riverside Drain intercepted all this flow, the $q_{\text {slug }}$ or $q_{\text {heat }}$ fluxes would only account for 18 to 21 percent, respectively, of the increase in flow in the drain.

The comparison of these results with those of previous investigations suggests that calculated flux through the Rio Grande inner valley alluvial aquifer is strongly scale dependent and that the thickness of aquifer through which river water flows may be greater than indicated by the vertical temperature profiles.

\section{Introduction}

The Albuquerque area (fig. 1) is the major population center in New Mexico and covers about 400 square miles $\left(\mathrm{mi}^{2}\right)$. With a population of approximately 535,000 people in 2000, the Albuquerque area accounts for 29 percent of the State's population (U.S. Census Bureau, 2000). Currently (2009), there are two principal sources of water for municipal, domestic, commercial, and industrial uses in this area: groundwater from the Santa Fe Group aquifer system, and surface water from the San Juan-Chama Diversion Project. The Rio Grande, which extends the length of New Mexico, is the principal source of water for irrigated agriculture (McAda, 1996). Estimates indicated that from 1960 to 2002, groundwater withdrawals from the Santa Fe Group aquifer system have caused water levels to decline more than 120 feet (ft) in some places within the Albuquerque area (Bexfield and Anderholm, 2002 and fig. 2). This has resulted in a great deal of interest in quantifying the riveraquifer interaction associated with the Rio Grande.

The aquifer system (middle Tertiary to Quaternary age) in the Albuquerque area consists of the Santa Fe Group aquifer and the post-Santa Fe Group (Quaternary age) Rio Grande inner valley alluvial aquifer (alluvial aquifer). The Santa Fe Group aquifer system is hydraulically connected to the Rio Grande (McAda, 1996) where fluvial gravel, sand, silt, and clay deposits form a thin but extensive aquifer zone below the Rio Grande flood plain (Hawley and Whitworth, 1996). The post-Santa Fe Group Rio Grande inner valley alluvial aquifer is composed of channel, flood plain, terrace, and tributary deposits that are as much as $120 \mathrm{ft}$ thick. Previous researchers have used streambed permeameters (Gould, 1994), the transient response of the aquifer to a flood pulse (Roark, 2001), vertical profiles of temperature measurements (Bartolino and Niswonger, 1999), and calibrated numerical models (Kernodle and others, 1995; Tiedeman and others, 1998) to estimate the flux between the Rio Grande and the Santa Fe Group aquifer system. Currently, a basin-scale groundwater model is used in the assessment of surface stream depletion (Barroll, 2001). In contrast to previous more regional-scale studies, a study was designed to focus on the upper alluvial aquifer and provide spatially detailed information about the amount of water that discharges from the Rio Grande to the adjacent aquifer in the Albuquerque area.

In 2003, the U.S. Geological Survey (USGS) in cooperation with the Bureau of Reclamation (BOR), the Middle Rio Grande Endangered Species Collaborative Program (MRGESCP), and the U.S. Army Corps of Engineers (USACE) began a detailed characterization of the hydrogeology of the Rio Grande riparian corridor in the Albuquerque, New Mexico, area to provide hydrologic data and enhance the understanding of rates of water leakage from the Rio Grande to the alluvial aquifer, groundwater flow through the aquifer, and discharge of water from the aquifer to the riverside drains. Beginning in late 2003 through 2006, a total of 10 east-west trending hydrologic transects were installed along both sides of the Rio Grande through the Albuquerque area at five selected locations (fig. 1). Each location consisted of paired transects of piezometers installed between the river and riverside drains to evaluate the rate of leakage from the river to riverside drains. In some cases (figs. 3a, 3c-3e), piezometers were installed at various distances outside the drains. Surface-water-stage gages also were installed in the river and in the east and west riverside drains. Lithologic information collected during drilling, and hourly groundwater-level and water-temperature data, and vertical temperature profile data collected during the study were used to define a conceptual model of flow in the Rio Grande inner valley alluvial aquifer adjacent to the river. Two methods are presented to quantify the rate of groundwater flux at depths less than $30 \mathrm{ft}$. In the first method, Darcy's law and estimates of hydraulic conductivity from slug tests and the literature are used to assess the variability in river leakage attributed to temporal changes in hydraulic gradient. In the second method, the Suzuki-Stallman one-dimensional analytical solution to the heat-transport equation are used to model annual groundwater-temperature changes within the aquifer resulting from river leakage. Temperature models provide additional detail on rates of groundwater flux with depth and distance from the river in the Albuquerque area.

\section{Purpose and Scope}

This report documents the collection and analysis of geologic, groundwater, and surface-water data from the inner valley of the Albuquerque area. The data are used to describe the groundwater hydrology of the Rio Grande inner valley alluvial aquifer and to estimate horizontal groundwater flux from the Rio Grande to the riverside drains. The report presents a simple conceptual model of groundwater flow and a comparison of groundwater-flux results derived from two methods: Darcy's law and the Suzuki-Stallman solution of heat transport in an aquifer. Data used in this report were collected from 10 transects located near the Paseo del Norte, Montaño, Barelas, Rio Bravo, and I-25 bridges (fig. 1). Groundwater and surface-water levels, temperature and slug-test data, seepage measurements, and core samples were collected from December 2003 to early 2009. 


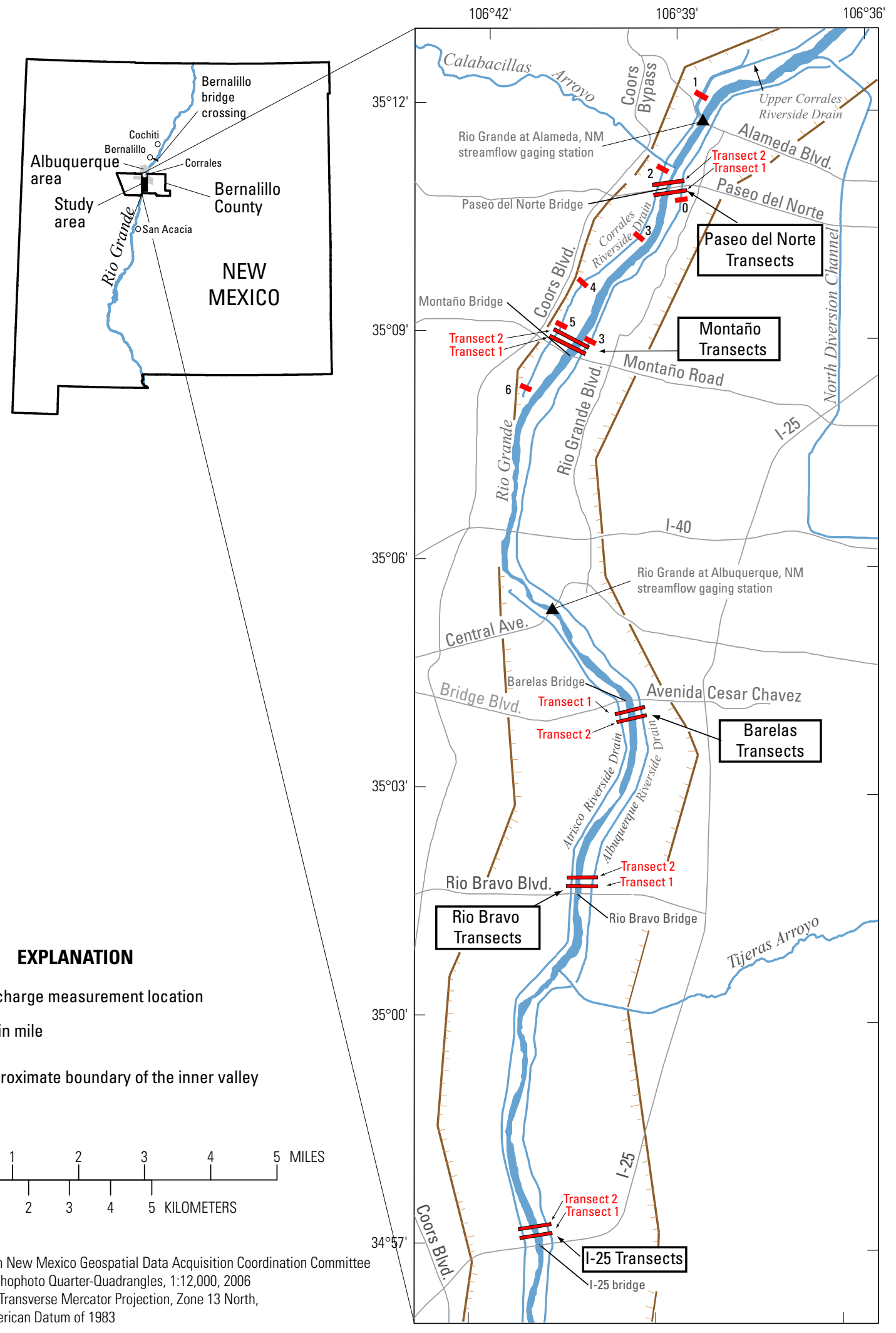

Figure 1. Location of study area and transects in the Albuquerque area, New Mexico. 


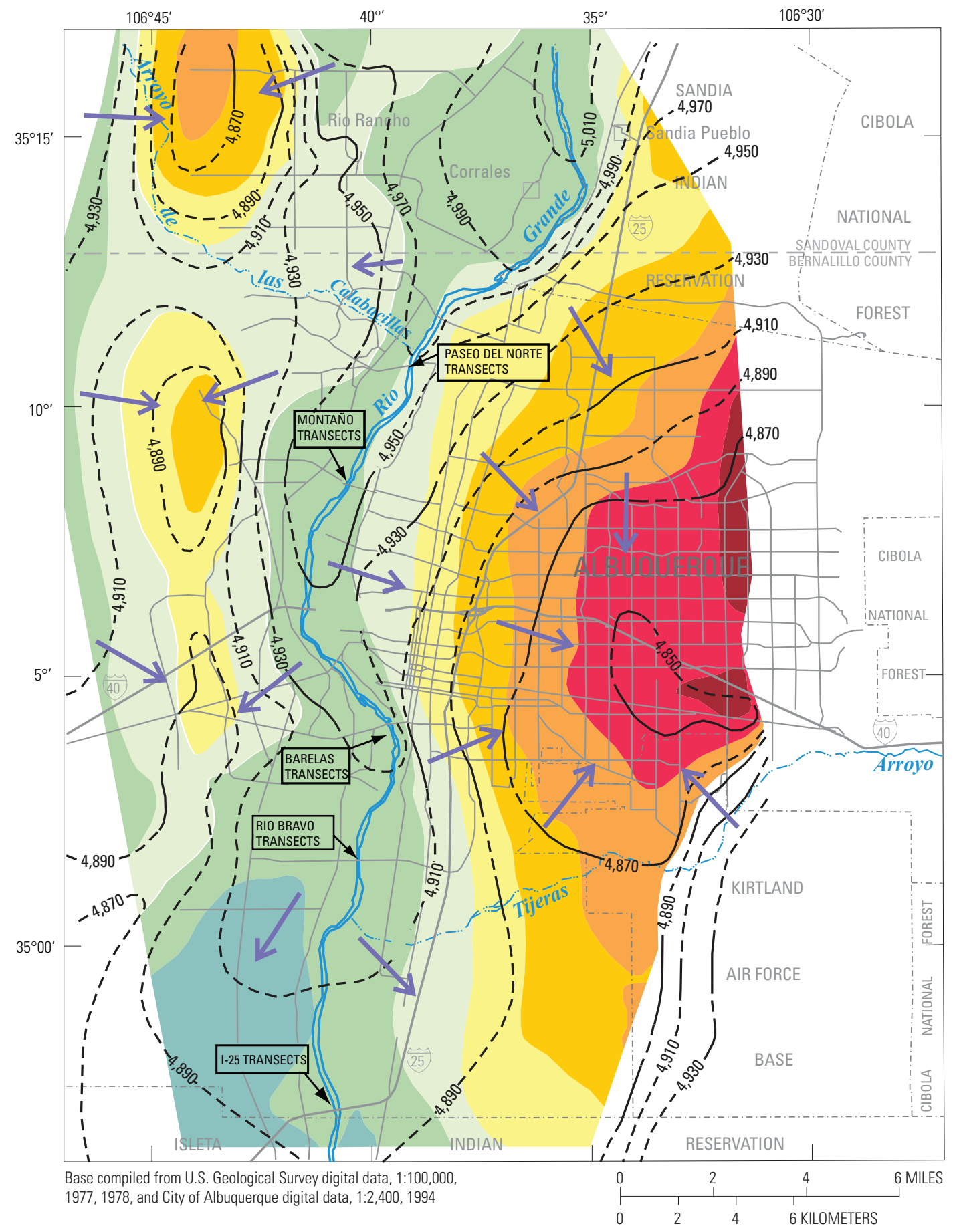

EXPLANATION

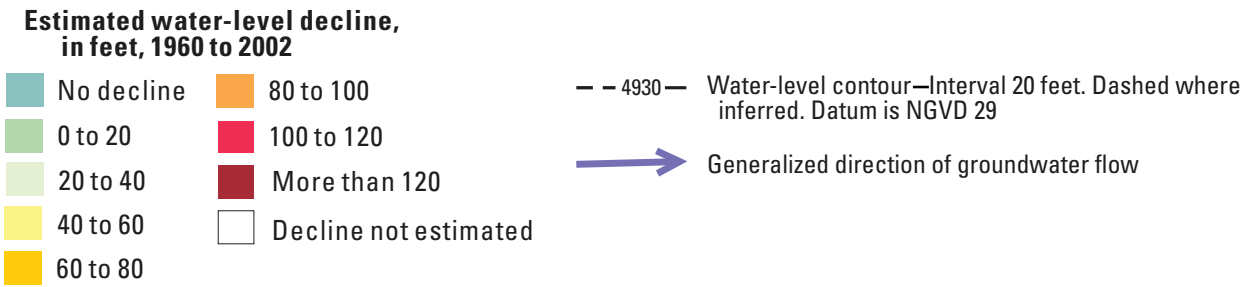

Figure 2. Groundwater-level-elevation contours in the Santa Fe Group aquifer in the Albuquerque area and estimated groundwaterlevel declines from 1960 to 2002. 


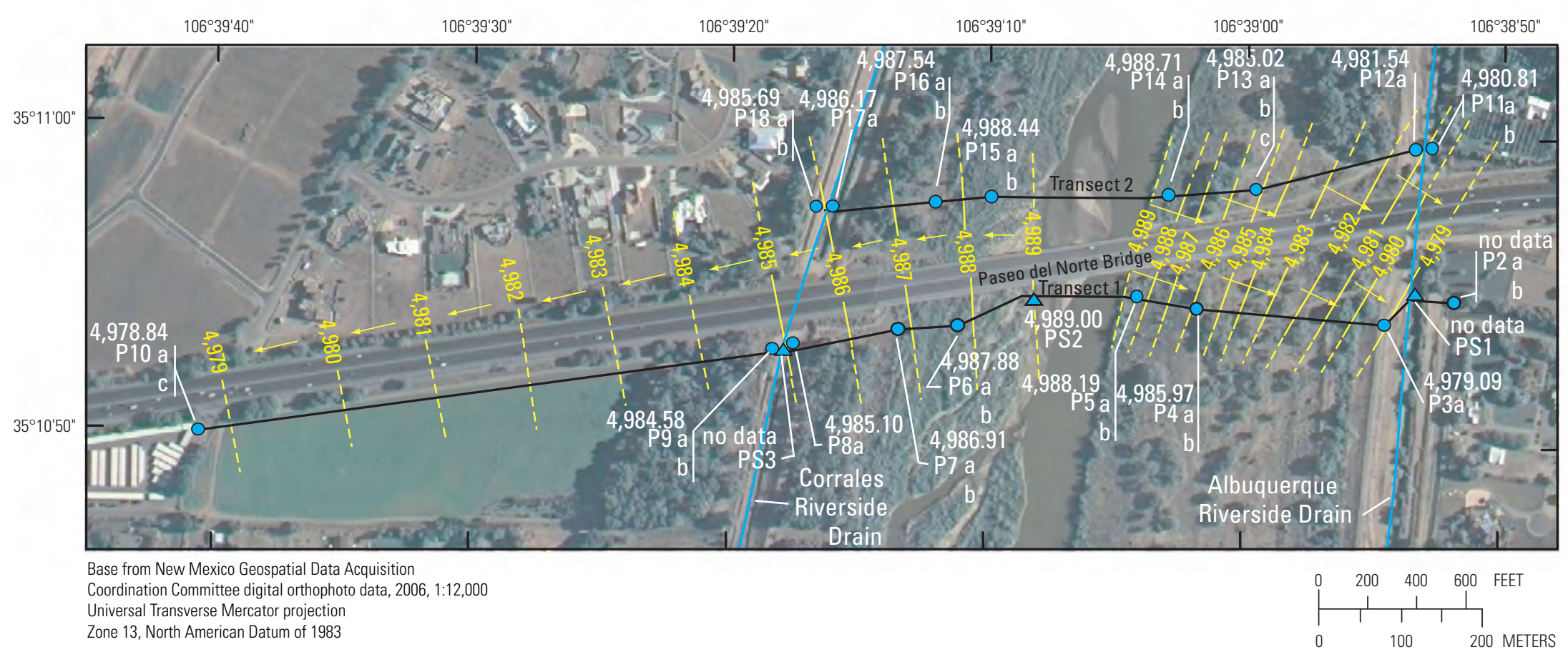

\section{EXPLANATION}

\section{Direction of groundwater flow}

\begin{tabular}{|c|c|}
\hline $4,980-$ & $\begin{array}{l}\text { Water-table contour-Interval is } 1 \text { foot. } \\
\text { Dashed where inferred. Datum is NAVD } 88\end{array}$ \\
\hline $\begin{array}{l}4,989.00 \\
\triangle P S 2\end{array}$ & $\begin{array}{l}\text { Surface-water gage, identifier, and mean daily } \\
\text { hydraulic head, in feet above NAVD } 88\end{array}$ \\
\hline $\begin{array}{l}4,985.02 \\
\text { O P13 }\end{array}$ & $\begin{array}{l}\text { Piezometer(s), identifiers(s), and mean daily } \\
\text { hydraulic head in shallow piezometer, in feet } \\
\text { above NAVD } 88\end{array}$ \\
\hline a & Shallow \\
\hline b & Mid-depth \\
\hline C & Deep \\
\hline
\end{tabular}

Figure 3a. Location of piezometer nests and surface-water gages and water-table contours showing direction of groundwater flow at the Paseo del Norte transects, 


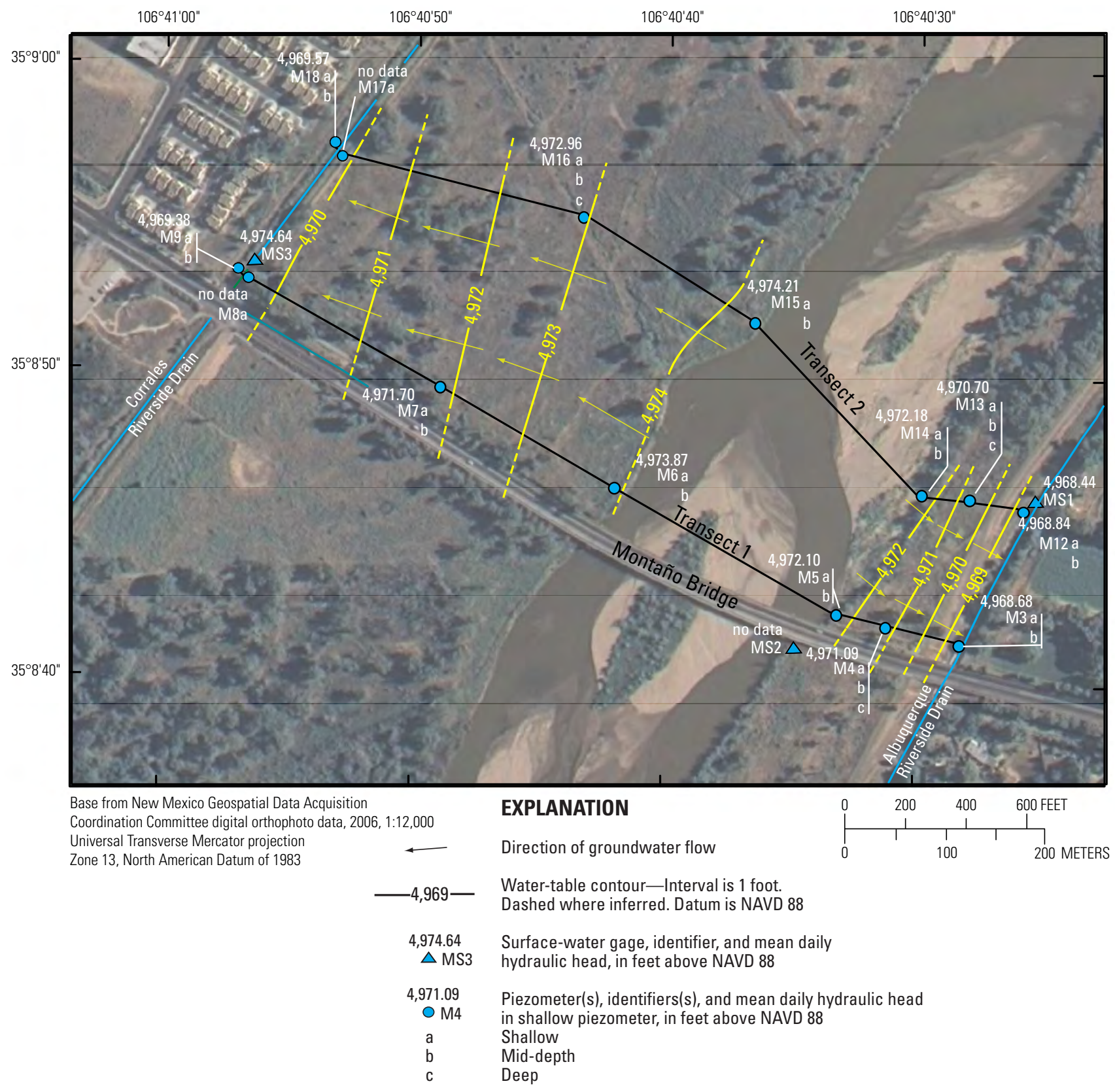

Figure $3 \mathbf{b}$. Location of piezometer nests and surface-water gages and water-table contours showing direction of groundwater flow at the Montaño transects, February 7, 2008. 


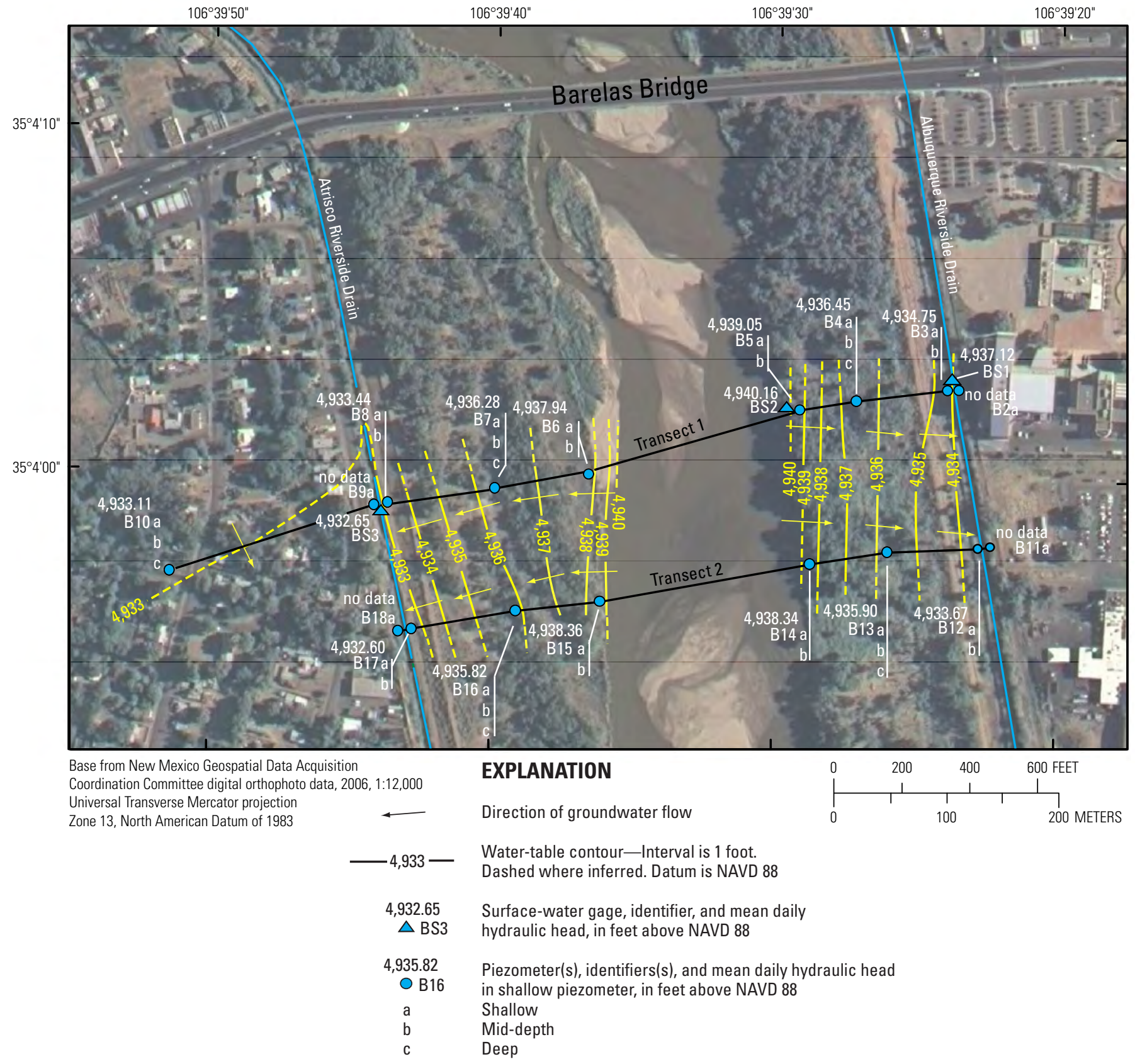

Figure 3c. Location of piezometer nests and surface-water gages and water-table contours showing direction of groundwater flow at the Barelas transects, February 7, 2008. 
$35^{\circ} 01^{\prime} 50^{\prime \prime}$

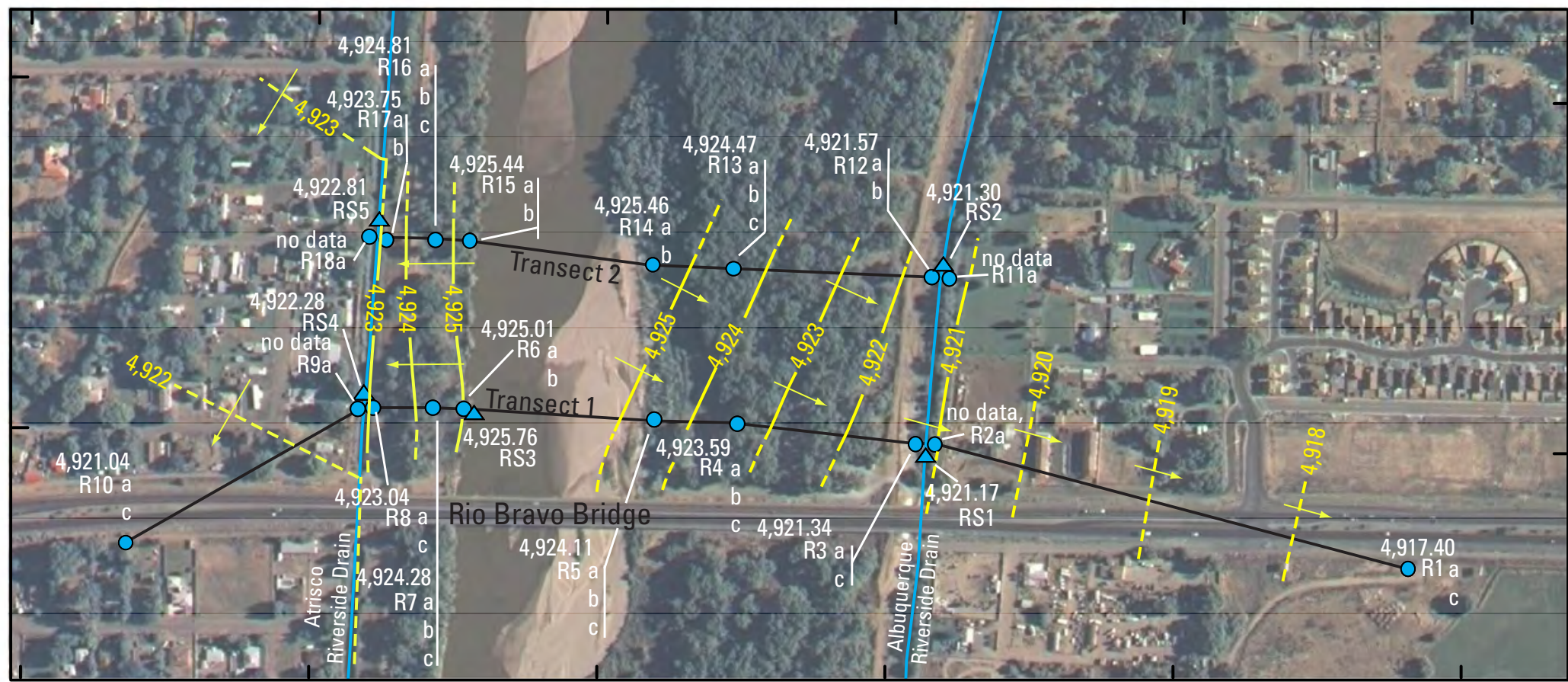

Base from New Mexico Geospatial Data Acquisition

Coordination Committee digital orthophoto data, 2006, 1:12,000

Universal Transverse Mercator projection

\section{EXPLANATION}

Zone 13, North American Datum of 1983
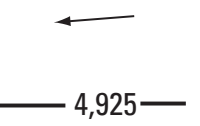

Direction of groundwater flow
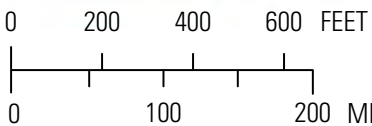

100

200 METERS

Water-table contour-Interval is 1 foot.

Dashed where inferred. Datum is NAVD 88

4,921.17 Surface-water gage, identifier, and mean daily

$\triangle \mathrm{RS1}$ hydraulic head, in feet above NAVD 88

4,924.11 Piezometer(s), identifiers(s), and mean daily hydraulic head in shallow piezometer, in feet above NAVD 88

Shallow

Mid-depth

Deep

Figure 3d. Location of piezometer nests and surface-water gages and water-table contours showing direction of groundwater flow at the Rio Bravo transects, February 7, 2008. 


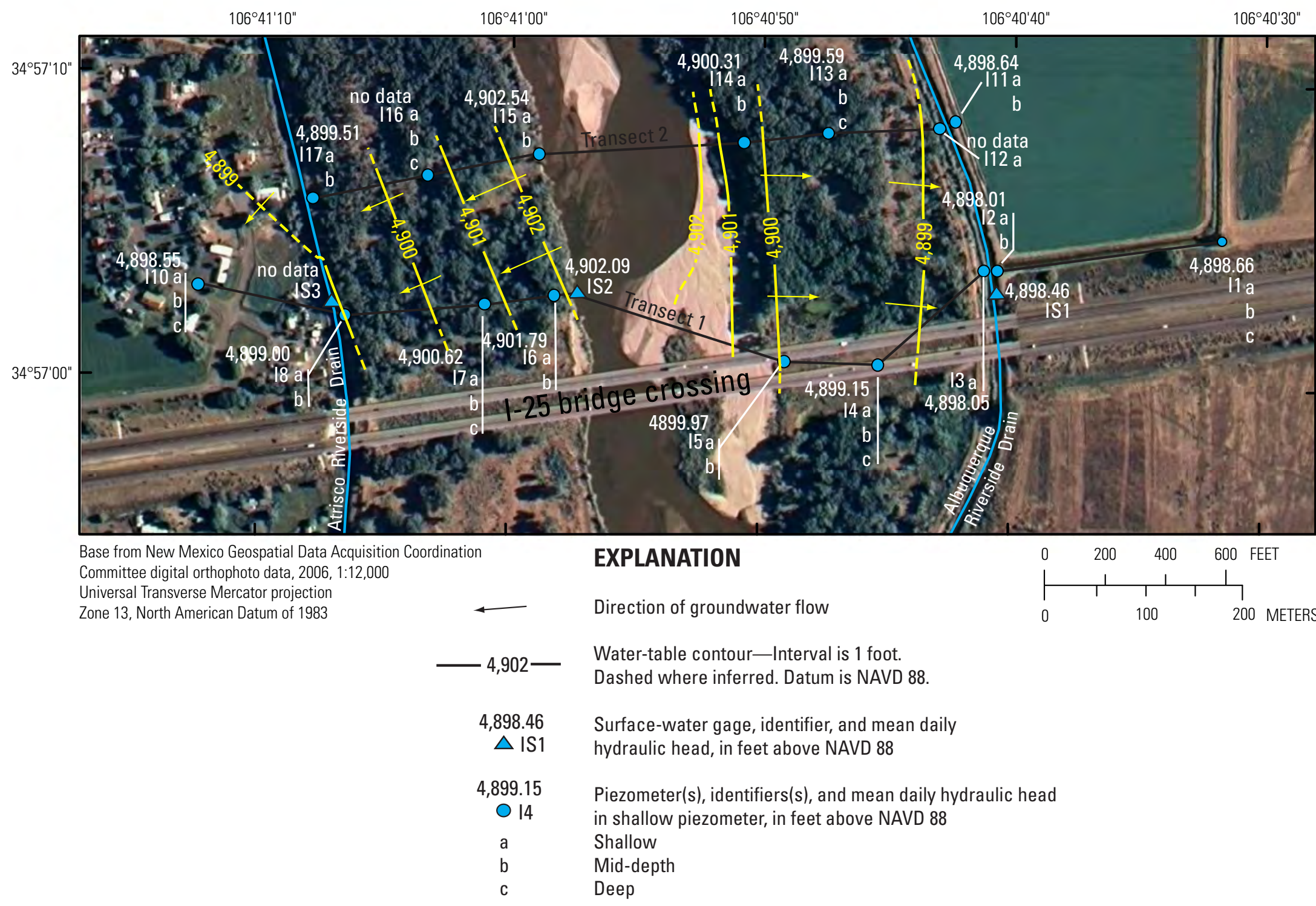

Figure 3e. Location of piezometer nests and surface-water gages and water-table contours showing direction of groundwater flow at the I-25 transects, February 7, 2008. 


\section{Description of the Study Area}

The study area extends about 18 miles (mi) along the Rio Grande in the Albuquerque area from the Paseo del Norte bridge to the I-25 bridge (fig. 1). The east and west edges of the study area are limited to areas within the inner valley adjacent to the Upper Corrales, Corrales, Albuquerque, and Atrisco Riverside Drains (fig. 1); the inner valley is approximately 2-3 mi wide and slopes about 5-6 feet per mile (ft/mi) southward through the Albuquerque area. The Rio Grande has a densely vegetated riparian area that supports a variety of biological communities. The riverside drains are ditches generally separated from the river by levees that are designed to intercept lateral groundwater flow from the river and prevent waterlogged-soil conditions east and west of the inner valley. Seepage to the riverside drains constitutes one of the main sources of groundwater discharge from the shallow alluvium (Kernodle and others, 1995).

\section{Rio Grande Inner Valley Alluvial Aquifer}

The Rio Grande inner valley alluvial aquifer consists of coarse-grained axial channel deposits and post-Santa Fe Group sediments that underlie the present-day Rio Grande flood plain (Hawley and Haase, 1992). In the Albuquerque area, the alluvium consists of unconsolidated to poorly consolidated, fine- to coarse-grain sand and rounded gravel with subordinate, discontinuous lens-shaped interbeds of fine-grain sand, silt, and clay (Connell and others, 2007). These deposits form an extensive shallow aquifer along the Rio Grande in the Albuquerque area and represent the last cut-and-fill cycle of the expansion of the Rio Grande fluvial system. Hawley and Haase (1992) indicate that these channel and flood-plain deposits may be as much as $120 \mathrm{ft}$ thick with an average thickness of $80 \mathrm{ft}$. Connell and others (2007) suggest that the inner valley probably was excavated during the Pleistocene epoch (about 1.8 million to 11,500 years before present) and subsequently was filled to near its present level by the middle Holocene epoch (about 8,000 to 5,000 years before present). The alluvial aquifer is overlain by and interfingers with valley border deposits of late Pleistocene and Holocene age derived from the major tributary drainages. The top of this unit has formed through deposition and includes the flood plain and channel of the Rio Grande.

\section{Santa Fe Group Aquifer System}

The Santa Fe Group aquifer system, which underlies the alluvial aquifer, is composed primarily of gravel, sand, silt, and clay. Most of these sediments were transported into faultbounded basins of the Rio Grande by rivers and drainages from surrounding areas (Bartolino and Cole, 2002). The spatial distribution of sedimentary facies of these deposits tends to be complex and three-dimensional rather than a simple, layered system (Bartolino and Cole, 2002).

Approximately 14,000 ft thick in parts of the basin, the Santa Fe Group is divided into upper, middle, and lower hydrostratigraphic units (Hawley and Haase, 1992). Sediments in the upper Santa Fe unit were deposited during the development of the ancestral Rio Grande and contain intertongued piedmont-slope and fluvial basin-floor deposits as thick as 1,200 ft (Hawley and Haase, 1992). Coarse-grain sediments comprise the ancestral Rio Grande axial-channel deposits contained in the upper unit of the Santa Fe Group. Sediments in the middle Santa Fe unit include piedmontslope deposits, fluvial basin-floor deposits, and basin-floor playa deposits (Hawley and Haase, 1992). This middle unit contains the largest accumulation of sediment and is as much as $10,000 \mathrm{ft}$ thick. Sediments in the lower Santa Fe unit are predominantly piedmont-slope, eolian, and basin-floor playa deposits and are as much as 3,500 ft thick (Hawley and Haase, 1992).

\section{Previous Investigations}

The interaction of groundwater and surface water in the Albuquerque area has been the focus of a number of investigations. McAda (1996) described the components of the Rio Grande and Santa Fe Group aquifer system in the Albuquerque area and prioritized activities to better understand groundwater and surface-water interaction. Peter (1987) compared differences in the configuration of the water table near the Rio Grande in the Albuquerque area from 1936 to 1986 and briefly described groundwater/surface-water interaction. Engdahl and others (2010) examined the effects of lithologic heterogeneity on the exchange of water between the surface and subsurface near the Rio Bravo bridge. Bartolino (2003) used groundwater levels and temperature data to evaluate groundwater fluxes in a single piezometer transect near the Paseo del Norte bridge. Bartolino and Niswonger (1999) measured groundwater-temperature profiles and groundwater levels near the Paseo del Norte and Rio Bravo bridges to simulate vertical groundwater flux and estimate vertical hydraulic conductivity. Bartolino and Sterling (2000) delineated specific areas on both sides of the river between the Paseo del Norte bridge and Rio Bravo bridge that contain hydrologically significant clay-rich layers.

The projected movement of groundwater in the Albuquerque area has been described by Kernodle and others (1995) and Bexfield and McAda (2003); the authors simulated historic and hypothetical groundwater flow in the Santa Fe Group aquifer system. Water-level declines in the Santa Fe Group aquifer have been estimated by Bexfield and Anderholm (2002); the authors report that contours of water-level data collected from 1999 to 2002 indicate that groundwater-flow direction in the Albuquerque 
area has changed significantly since 1961 because of increased pumping.

McAda and Barroll (2002) simulated groundwater flow using a three-dimensional groundwater-flow model of the Santa Fe Group aquifer system from Cochiti to San Acacia. Sanford and others (2003) used environmental tracers to estimate aquifer parameters for a predevelopment groundwater-flow model in the Middle Rio Grande Basin.

Kues (1986) described the movement of shallow groundwater near the Rio Grande between the Barelas bridge and the I- 25 bridge based on single measurements of water levels from 44 wells. Anderholm and Bullard (1987) described the installation of piezometers in the Albuquerque area and provided lithologic descriptions from monitoring wells drilled along Rio Bravo Boulevard and Montaño Road. Roark (2001) evaluated river flood pulses to estimate hydraulic characteristics of the Santa Fe Group aquifer system.

In general, the studies discussed above have either been very site-specific (Bartolino and Niswonger, 1999) or have been more regional in nature (McAda and Barroll, 2002). The study described in this report was designed to provide geohydrologic data and interpretations at a higher spatial resolution over a longer reach of the Rio Grande than has been provided by previous studies. Additionally, this study focuses on the geohydrology of the upper part of the alluvial aquifer.

\section{Methods of Data Collection and Analysis}

\section{Piezometer Installation and Core Descriptions}

Piezometers and surface-water gages were installed in paired transects near five bridges in the Albuquerque area (fig. 1, table 1). Each transect included nested piezometers (multiple monitoring wells with screen openings at different depths) and surface-water-stage gages configured in roughly straight lines and oriented perpendicular to the river and riverside drains (fig. 3). At each location, transects extended from the Rio Grande to just outside the riverside drains on both sides of the river and were spaced about $500 \mathrm{ft}$ apart. The paired-transect configuration was chosen to facilitate definition of horizontal and vertical gradients at each location.

Piezometer nests generally were installed with the deep piezometer screen at 45 to $50 \mathrm{ft}$ and the mid-depth piezometer screen at 30 to $35 \mathrm{ft}$. The shallow piezometer screen typically was installed at 5 to $10 \mathrm{ft}$ to intersect the expected range in seasonal depths to the water table. The deep piezometer was installed first. The water level measured in the deep piezometer was then used to determine the depths for the mid-depth and shallow piezometer screens. Each piezometer is labeled - the capital letter and number indicate location, and the small letter indicates piezometer depth (a, shallow; b, middepth; and c, deep). Each surface-water gage is labeled - the first capital letter and number indicate location, and the second capital letter (S) indicates a surface-water gage (fig. 3).

Piezometers were installed using direct-push drilling technology. Piezometers were constructed of 1-inch diameter, flush-threaded schedule 40 polyvinyl chloride (PVC) pipe. Each piezometer, from the bottom up, consists of a 5 - $\mathrm{ft}$ long blank section of casing capped at the bottom (the sump), a 5-ft long screen with 0.010-inch wide screen slots, and blank casing to the land surface. Each piezometer was completed by backfilling the outside annulus surrounding the PVC with soil to a depth of about $5 \mathrm{ft}$ below land surface. Bentonite pellets were then placed in the annulus from the top of the backfill to land surface. Each piezometer was developed using compressed air to pump water out of the casing.

Continuous subsurface core samples were collected at each transect from 2004 to 2006 by using direct-push drilling technology. Core samples were obtained to (1) ensure that piezometer screens were placed in sand and gravel, and not clay; and, (2) identify and locate any substantial changes in subsurface lithology that could potentially affect either horizontal or vertical groundwater movement. In total, 36 locations were cored within the study area. Coring locations generally corresponded to piezometer sites and included locations adjacent to the river, between the river and riverside drains on both sides of the river, and adjacent to both riverside drains. Coring depths ranged from 25 to $55 \mathrm{ft}$, depending on the depth of the deepest piezometer.

Cores were collected in acetate tubes; each tube was capped and labeled. Cores were described in the field at the time of collection. Core descriptions include the depth interval that was cored, the amount of recovery from each interval, the lithology (grain size, sorting, rock type, and color), and miscellaneous remarks.

\section{Water-Level and Temperature Data}

Groundwater and surface-water levels were measured in piezometers and surface-water bodies for this study. Submersible water-pressure sensors (transducers) were installed in each piezometer to measure and record hourly groundwater levels; some of the transducers also were capable of recording hourly temperatures. Surface-water-stage gages were constructed to measure and record water levels in the Rio Grande and in the riverside drains; streamflow was not computed for these gages. A total of 164 piezometers and 17 surface-water gages were installed; the number of piezometers and surface-water gages in each set of transects varied for each location. 
[S, surface-water stage gage; B, Barelas; I, I-25; M, Montaño; P, Paseo del Norte; R, Rio Bravo; a, shallow piezometer; b, mid-depth piezometer; c, deep piezometer; MP, measuring point; na, not applicable; NAVD 88, North American Vertical Datum of 1988]

\begin{tabular}{|c|c|c|c|c|c|c|c|}
\hline \multirow{2}{*}{$\begin{array}{c}\text { Other } \\
\text { identifier } \\
\text { (fig. 3a-e) }\end{array}$} & \multirow[b]{2}{*}{ Site identifier } & \multirow{2}{*}{$\begin{array}{l}\text { Piezometer depth } \\
\text { (feet below land } \\
\text { surface, rounded } \\
\text { to nearest foot) }\end{array}$} & \multirow{2}{*}{$\begin{array}{l}\text { Screened interval } \\
\text { (feet below land } \\
\text { surface, rounded to } \\
\text { nearest foot) }\end{array}$} & \multirow{2}{*}{$\begin{array}{c}\text { Land surface }^{1} \\
\text { or MP2 elevation } \\
\text { (feet above NAVD 88) }\end{array}$} & \multicolumn{2}{|c|}{$\begin{array}{c}\text { Period of record presented in this } \\
\text { report }\end{array}$} & \multirow{2}{*}{$\begin{array}{l}\text { Type of transducer in well } \\
\text { for period of record } \\
\text { presented in this report }\end{array}$} \\
\hline & & & & & Start Date & End Date & \\
\hline \multicolumn{8}{|c|}{ Paseo del Norte transects } \\
\hline $\mathrm{P} 2 \mathrm{a}$ & 351055106385101 & 22 & $12-17$ & 4994.53 & \multicolumn{2}{|c|}{ Uninstrumented during study } & na \\
\hline $\mathrm{P} 2 \mathrm{~b}$ & 351055106385102 & 40 & $30-35$ & 4994.53 & $11 / 07 / 2007$ & 03/04/2009 & Vented \\
\hline P3a & 351054106385401 & 25 & $15-20$ & 4992.11 & $11 / 07 / 2007$ & 03/04/2009 & Vented \\
\hline $\mathrm{P} 4 \mathrm{a}$ & 351054106390101 & 16 & $6-11$ & 4993.29 & $11 / 07 / 2007$ & 03/04/2009 & Vented \\
\hline $\mathrm{P} 4 \mathrm{~b}$ & 351054106390102 & 35 & $25-30$ & 4993.29 & $11 / 07 / 2007$ & 03/04/2009 & Vented \\
\hline $\mathrm{P} 5 \mathrm{a}$ & 351054106390401 & 16 & $6-11$ & 4993.53 & $11 / 07 / 2007$ & 03/04/2009 & Vented \\
\hline $\mathrm{P} 5 \mathrm{~b}$ & 351054106390402 & 35 & $25-30$ & 4993.53 & $11 / 07 / 2007$ & 03/04/2009 & Vented \\
\hline P6a & 351055106391101 & 16 & $6-11$ & 4992.03 & $11 / 07 / 2007$ & 03/04/2009 & Unvented \\
\hline P6b & 351055106391102 & 31 & $21-26$ & 4992.03 & $11 / 07 / 2007$ & 03/04/2009 & Unvented \\
\hline $\mathrm{P} 7 \mathrm{a}$ & 351054106391301 & 16 & $6-11$ & 4993.47 & $11 / 07 / 2007$ & 03/04/2009 & Unvented \\
\hline $\mathrm{P} 7 \mathrm{~b}$ & 351054106391302 & 31 & $21-26$ & 4993.47 & $11 / 07 / 2007$ & 03/04/2009 & Unvented \\
\hline P8a & 351052106391701 & 16 & $6-11$ & 4990.86 & $11 / 07 / 2007$ & 03/04/2009 & Unvented \\
\hline P9a & 351053106391701 & 23 & $13-18$ & 4996.34 & $11 / 07 / 2007$ & 03/04/2009 & Unvented \\
\hline $\mathrm{P} 9 \mathrm{~b}$ & 351053106391702 & 27 & $17-22$ & 4996.34 & $11 / 07 / 2007$ & 03/04/2009 & Unvented \\
\hline $\mathrm{P} 10 \mathrm{a}$ & 351050106394001 & 22 & $12-17$ & 4991.09 & $11 / 13 / 2007$ & 03/04/2009 & Vented \\
\hline $\mathrm{P} 10 \mathrm{c}$ & 351050106394002 & 46 & $36-41$ & 4991.09 & \multicolumn{2}{|c|}{ Uninstrumented during study } & na \\
\hline P11a & 351059106385201 & 22 & $12-17$ & 4991.37 & $10 / 17 / 2008$ & 03/03/2009 & Unvented \\
\hline $\mathrm{P} 11 \mathrm{~b}$ & 351059106385202 & 37 & $27-32$ & 4991.37 & $10 / 17 / 2008$ & 03/03/2009 & Unvented \\
\hline $\mathrm{P} 12 \mathrm{a}$ & 351059106385301 & 22 & $12-17$ & 4991.77 & $10 / 17 / 2008$ & 03/03/2009 & Unvented \\
\hline P13a & 351058106385901 & 17 & $7-12$ & 4993.36 & $10 / 17 / 2008$ & 03/03/2009 & Unvented \\
\hline $\mathrm{P} 13 \mathrm{~b}$ & 351058106385902 & 32 & $22-27$ & 4993.36 & $10 / 17 / 2008$ & 03/03/2009 & Unvented \\
\hline $\mathrm{P} 13 \mathrm{c}$ & 351058106385903 & 52 & $42-47$ & 4993.36 & $10 / 17 / 2008$ & 03/03/2009 & Unvented \\
\hline P14a & 351058106390301 & 15 & $5-10$ & 4993.76 & $10 / 17 / 2008$ & 03/03/2009 & Unvented \\
\hline $\mathrm{P} 14 \mathrm{~b}$ & 351058106390302 & 30 & $20-25$ & 4993.76 & $10 / 17 / 2008$ & 03/03/2009 & Unvented \\
\hline P15a & 351058106391001 & 16 & $6-11$ & 4992.33 & $10 / 15 / 2008$ & 03/03/2009 & Unvented \\
\hline $\mathrm{P} 15 \mathrm{~b}$ & 351058106391002 & 31 & $21-26$ & 4992.33 & $10 / 15 / 2008$ & 03/03/2009 & Unvented \\
\hline
\end{tabular}




\begin{tabular}{|c|c|c|c|c|c|c|c|}
\hline \multirow{2}{*}{$\begin{array}{l}\text { Other } \\
\text { identifier } \\
\text { (fig. 3a-e) }\end{array}$} & \multirow[b]{2}{*}{ Site identifier } & \multirow{2}{*}{$\begin{array}{l}\text { Piezometer depth } \\
\text { (feet below land } \\
\text { surface, rounded } \\
\text { to nearest foot) }\end{array}$} & \multirow{2}{*}{$\begin{array}{l}\text { Screened interval } \\
\text { (feet below land } \\
\text { surface, rounded to } \\
\text { nearest foot) }\end{array}$} & \multirow{2}{*}{$\begin{array}{c}\text { Land surface }^{1} \\
\text { or } \mathrm{MP}^{2} \text { elevation } \\
\text { (feet above NAVD 88) }\end{array}$} & \multicolumn{2}{|c|}{$\begin{array}{c}\text { Period of record presented in this } \\
\text { report }\end{array}$} & \multirow{2}{*}{$\begin{array}{l}\text { Type of transducer in well } \\
\text { for period of record } \\
\text { presented in this report }\end{array}$} \\
\hline & & & & & Start Date & End Date & \\
\hline \multicolumn{8}{|c|}{ Paseo del Norte transects-Continued } \\
\hline P16a & 351058106391101 & 16 & $6-11$ & 4993.26 & $10 / 15 / 2008$ & 03/03/2009 & Unvented \\
\hline $\mathrm{P} 16 \mathrm{~b}$ & 351058106391102 & 40 & $30-35$ & 4993.26 & $10 / 15 / 2008$ & 03/03/2009 & Unvented \\
\hline P17a & 351058106391501 & 16 & $6-11$ & 4989.73 & $10 / 15 / 2008$ & 03/03/2009 & Unvented \\
\hline P18a & 351058106391601 & 20 & $10-15$ & 4991.50 & $10 / 15 / 2008$ & 03/03/2009 & Unvented \\
\hline $\mathrm{P} 18 \mathrm{~b}$ & 351058106391602 & 35 & $25-30$ & 4991.50 & $10 / 15 / 2008$ & 03/03/2009 & Unvented \\
\hline PS1 & 351054106385310 & na & na & 4984.21 & $10 / 17 / 2008$ & 03/04/2009 & Unvented \\
\hline PS2 & 351055106390810 & na & na & 4993.49 & $10 / 15 / 2008$ & 03/04/2009 & Unvented \\
\hline PS3 & 351053106391710 & na & na & 4989.62 & $10 / 15 / 2008$ & 03/04/2009 & Unvented \\
\hline \multicolumn{8}{|c|}{ Montaño transects } \\
\hline M3a & 350843106402801 & 17 & $7-12$ & 4974.14 & $12 / 05 / 2005$ & $10 / 31 / 2008$ & Unvented \\
\hline M3b & 350843106402802 & 31 & $21-26$ & 4974.14 & $12 / 05 / 2005$ & $08 / 22 / 2006$ & Unvented \\
\hline M4a & 350842106403101 & 15 & $5-10$ & 4978.85 & 01/09/2006 & $10 / 31 / 2008$ & Unvented \\
\hline M4b & 350842106403102 & 30 & $20-25$ & 4978.85 & $06 / 13 / 2005$ & $10 / 31 / 2008$ & Unvented \\
\hline M4c & 350842106403103 & 49 & $39-44$ & 4978.85 & $06 / 13 / 2005$ & $10 / 31 / 2008$ & Unvented \\
\hline M5a & 350842106403201 & 15 & $5-10$ & 4978.20 & $01 / 09 / 2006$ & $10 / 31 / 2008$ & Unvented \\
\hline M5b & 350842106403202 & 32 & $22-27$ & 4978.20 & $06 / 13 / 2005$ & $10 / 31 / 2008$ & Unvented \\
\hline M6a & 350848106404703 & 13 & $3-8$ & 4978.86 & $10 / 12 / 2005$ & $10 / 31 / 2008$ & Unvented \\
\hline M6b & 350848106404704 & 28 & $18-23$ & 4978.86 & $10 / 12 / 2005$ & $10 / 31 / 2008$ & Unvented \\
\hline M7a & 350848106404701 & 15 & $5-10$ & 4977.89 & $10 / 12 / 2005$ & $10 / 31 / 2008$ & Unvented \\
\hline $\mathrm{M} 7 \mathrm{~b}$ & 350848106404702 & 30 & $20-25$ & 4977.89 & $10 / 12 / 2005$ & $10 / 31 / 2008$ & Unvented \\
\hline M8a & 350852106405601 & 16 & $6-11$ & 4979.78 & $10 / 12 / 2005$ & $01 / 24 / 2007$ & Unvented \\
\hline M9a & 350853106405701 & 15 & $5-10$ & 4977.39 & $10 / 12 / 2005$ & $10 / 31 / 2008$ & Unvented \\
\hline M9b & 350853106405702 & 30 & $20-25$ & 4977.39 & $06 / 13 / 2005$ & $10 / 31 / 2008$ & Unvented \\
\hline M12a & 350847106402501 & 17 & $7-12$ & 4977.26 & 01/09/2006 & $11 / 03 / 2008$ & Unvented \\
\hline M12b & 350847106402502 & 32 & $22-27$ & 4977.26 & $01 / 09 / 2006$ & $10 / 31 / 2008$ & $\begin{array}{c}\text { Unvented }(01 / 09 / 2006- \\
08 / 22 / 2006), \text { vented }(03 / 16 / 2007 \\
-10 / 31 / 2008)\end{array}$ \\
\hline M13a & 350846106402801 & 16 & $6-11$ & 4977.37 & 03/16/2007 & $10 / 31 / 2008$ & Vented \\
\hline M13b & 350846106402802 & 31 & $21-26$ & 4977.37 & $01 / 09 / 2006$ & $10 / 31 / 2008$ & Unvented \\
\hline M13c & 350846106402803 & 47 & $37-42$ & 4977.37 & $01 / 09 / 2006$ & $10 / 31 / 2008$ & Unvented \\
\hline
\end{tabular}


Table 1. Site data for piezometers and surface-water data collection sites, Rio Grande inner valley, Albuquerque, New Mexico.-Continued

[S, surface-water stage gage; B, Barelas; I, I-25; M, Montaño; P, Paseo del Norte; R, Rio Bravo; a, shallow piezometer; b, mid-depth piezometer; c, deep piezometer; MP, measuring point; na, not applicable; NAVD 88, North American Vertical Datum of 1988]

\begin{tabular}{|c|c|c|c|c|c|c|c|}
\hline \multirow{2}{*}{$\begin{array}{c}\text { Other } \\
\text { identifier } \\
\text { (fig. 3a-e) }\end{array}$} & \multirow[b]{2}{*}{ Site identifier } & \multirow{2}{*}{$\begin{array}{l}\text { Piezometer depth } \\
\text { (feet below land } \\
\text { surface, rounded } \\
\text { to nearest foot) }\end{array}$} & \multirow{2}{*}{$\begin{array}{l}\text { Screened interval } \\
\text { (feet below land } \\
\text { surface, rounded to } \\
\text { nearest foot) }\end{array}$} & \multirow{2}{*}{$\begin{array}{c}\text { Land surface }^{1} \\
\text { or MP² elevation } \\
\text { (feet above NAVD 88) }\end{array}$} & \multicolumn{2}{|c|}{$\begin{array}{c}\text { Period of record presented in this } \\
\text { report }\end{array}$} & \multirow{2}{*}{$\begin{array}{l}\text { Type of transducer in wel } \\
\text { for period of record } \\
\text { presented in this report }\end{array}$} \\
\hline & & & & & Start Date & End Date & \\
\hline \multicolumn{8}{|c|}{ Montaño transects-Continued } \\
\hline M14a & 350846106402804 & 18 & $8-13$ & 4979.83 & $11 / 17 / 2006$ & $10 / 31 / 2008$ & Vented \\
\hline M14b & 350846106402805 & 33 & $23-28$ & 4979.83 & $01 / 13 / 2006$ & $04 / 29 / 2008$ & Vented \\
\hline M15a & 350851106403801 & 13 & $3-8$ & 4977.80 & $02 / 01 / 2006$ & $10 / 31 / 2008$ & Vented \\
\hline M15b & 350851106403802 & 28 & $18-23$ & 4977.80 & $02 / 01 / 2006$ & $10 / 31 / 2008$ & Vented \\
\hline M16a & 350854106404201 & 17 & $7-12$ & 4978.62 & $02 / 01 / 2006$ & $10 / 31 / 2008$ & Vented \\
\hline M16b & 350854106404202 & 32 & $22-27$ & 4978.62 & $02 / 01 / 2006$ & $10 / 31 / 2008$ & Vented \\
\hline M16c & 350854106404203 & 46 & $36-41$ & 4978.62 & $02 / 01 / 2006$ & $10 / 31 / 2008$ & Vented \\
\hline M17a & 350855106405401 & 18 & 8-13 & 4978.88 & $02 / 01 / 2006$ & $01 / 26 / 2007$ & Vented \\
\hline M18a & 350857106405401 & 18 & $8-13$ & 4978.17 & $02 / 01 / 2006$ & $10 / 31 / 2008$ & Vented \\
\hline M18b & 350857106405402 & 33 & $23-28$ & 4978.17 & $02 / 01 / 2006$ & $10 / 31 / 2008$ & Vented \\
\hline MS1 & 350846106402510 & na & na & 4972.61 & $06 / 20 / 2006$ & $10 / 31 / 2008$ & Vented \\
\hline MS2 & 350841106403510 & na & na & 4978.52 & $06 / 20 / 2006$ & $07 / 22 / 2008$ & Vented \\
\hline MS3 & 350854106405610 & na & na & 4979.11 & $06 / 20 / 2006$ & $10 / 31 / 2008$ & Vented \\
\hline $\mathrm{B} 2 \mathrm{a}$ & 350403106392201 & 16 & $6-11$ & 4940.68 & $10 / 30 / 2008$ & 03/10/2009 & Unvented \\
\hline \multicolumn{8}{|c|}{ Barelas transects } \\
\hline B3a & 350403106392301 & 15 & $5-10$ & 4941.86 & $10 / 07 / 2005$ & 03/10/2009 & Unvented \\
\hline $\mathrm{B} 3 \mathrm{~b}$ & 350403106392302 & 30 & $20-25$ & 4941.86 & $06 / 13 / 2005$ & 03/10/2009 & Unvented \\
\hline $\mathrm{B} 4 \mathrm{a}$ & 350402106392601 & 16 & 6-11 & 4942.18 & $10 / 07 / 2005$ & 03/10/2009 & Unvented \\
\hline $\mathrm{B} 4 \mathrm{~b}$ & 350402106392602 & 31 & $21-26$ & 4942.18 & $06 / 13 / 2005$ & $10 / 30 / 2008$ & Unvented \\
\hline $\mathrm{B} 4 \mathrm{c}$ & 350402106392603 & 52 & $42-47$ & 4942.18 & $06 / 13 / 2005$ & 03/10/2009 & Unvented \\
\hline B5a & 350402106392901 & 15 & $5-10$ & 4943.08 & $10 / 07 / 2005$ & 03/10/2009 & Unvented \\
\hline $\mathrm{B} 5 \mathrm{~b}$ & 350402106392902 & 30 & $20-25$ & 4943.08 & $06 / 13 / 2005$ & $10 / 30 / 2008$ & Unvented \\
\hline B6a & 350400106393701 & 17 & $7-12$ & 4942.40 & $10 / 07 / 2005$ & 03/10/2009 & Unvented \\
\hline B6b & 350400106393702 & 32 & $22-27$ & 4942.40 & $06 / 13 / 2005$ & 03/10/2009 & Unvented \\
\hline B7a & 350359106393901 & 17 & $7-12$ & 4943.03 & $10 / 07 / 2005$ & 03/10/2009 & Unvented \\
\hline B $7 b$ & 350359106393902 & 32 & $22-27$ & 4943.03 & $04 / 21 / 2005$ & $10 / 22 / 2008$ & Unvented \\
\hline B7c & 350359106393903 & 52 & $42-47$ & 4943.03 & $04 / 21 / 2005$ & 03/10/2009 & Unvented \\
\hline B8a & 350359106394401 & 16 & $6-11$ & 4940.23 & $10 / 05 / 2005$ & 03/10/2009 & Unvented \\
\hline $\mathrm{B} 8 \mathrm{~b}$ & 350359106394402 & 34 & $24-29$ & 4940.23 & $04 / 21 / 2005$ & $10 / 22 / 2008$ & Unvented \\
\hline
\end{tabular}




\begin{tabular}{|c|c|c|c|c|c|c|c|}
\hline \multirow{2}{*}{$\begin{array}{c}\text { Other } \\
\text { identifier } \\
\text { (fig. 3a-e) }\end{array}$} & \multirow[b]{2}{*}{ Site identifier } & \multirow{2}{*}{$\begin{array}{l}\text { Piezometer depth } \\
\text { (feet below land } \\
\text { surface, rounded } \\
\text { to nearest foot) }\end{array}$} & \multirow{2}{*}{$\begin{array}{l}\text { Screened interval } \\
\text { (feet below land } \\
\text { surface, rounded to } \\
\text { nearest foot) }\end{array}$} & \multirow{2}{*}{$\begin{array}{c}\text { Land surface }^{1} \\
\text { or MP2 elevation } \\
\text { (feet above NAVD 88) }\end{array}$} & \multicolumn{2}{|c|}{$\begin{array}{c}\text { Period of record presented in this } \\
\text { report }\end{array}$} & \multirow{2}{*}{$\begin{array}{l}\text { Type of transducer in well } \\
\text { for period of record } \\
\text { presented in this report }\end{array}$} \\
\hline & & & & & Start Date & End Date & \\
\hline \multicolumn{8}{|c|}{ Barelas transects-Continued } \\
\hline B9a & 350359106394501 & 20 & $10-15$ & 4943.21 & \multicolumn{2}{|c|}{ Uninstrumented during study } & na \\
\hline B10a & 350354106395201 & 17 & $7-12$ & 4940.77 & $06 / 27 / 2005$ & 03/10/2009 & Unvented \\
\hline $\mathrm{B} 10 \mathrm{~b}$ & 350354106395202 & 32 & $22-27$ & 4940.77 & $07 / 13 / 2005$ & 03/10/2009 & Unvented \\
\hline $\mathrm{B} 10 \mathrm{c}$ & 350354106395203 & 48 & $38-43$ & 4940.77 & $07 / 13 / 2005$ & 03/10/2009 & Unvented \\
\hline B11a & 350358106392201 & 16 & $6-11$ & 4939.91 & $10 / 31 / 2008$ & 03/10/2009 & Unvented \\
\hline B12a & 350358106392301 & 16 & $6-11$ & 4939.88 & $02 / 01 / 2006$ & 03/10/2009 & Vented \\
\hline $\mathrm{B} 12 \mathrm{~b}$ & 350358106392302 & 31 & $21-26$ & 4939.88 & $02 / 01 / 2006$ & 03/10/2009 & Vented \\
\hline B13a & 350358106392601 & 15 & $5-10$ & 4941.91 & $01 / 30 / 2006$ & 03/10/2009 & Vented \\
\hline $\mathrm{B} 13 \mathrm{~b}$ & 350358106392602 & 30 & $20-25$ & 4941.91 & $01 / 30 / 2006$ & 03/10/2009 & Vented \\
\hline $\mathrm{B} 13 \mathrm{c}$ & 350358106392603 & 40 & $30-35$ & 4941.91 & $01 / 30 / 2006$ & 03/10/2009 & Vented \\
\hline B14a & 350357106392901 & 15 & $5-10$ & 4943.41 & $01 / 30 / 2006$ & 03/10/2009 & Vented \\
\hline $\mathrm{B} 14 \mathrm{~b}$ & 350357106392902 & 30 & $20-25$ & 4943.41 & $01 / 30 / 2006$ & 03/10/2009 & Vented \\
\hline B15a & 350356106393601 & 16 & $6-11$ & 4943.14 & $01 / 30 / 2006$ & $10 / 22 / 2008$ & Vented \\
\hline $\mathrm{B} 15 \mathrm{~b}$ & 350356106393602 & 31 & $21-26$ & 4943.14 & $01 / 30 / 2006$ & $01 / 15 / 2008$ & Vented \\
\hline B16a & 350356106393901 & 16 & $6-11$ & 4943.59 & $01 / 30 / 2006$ & 03/10/2009 & Vented \\
\hline B16b & 350356106393902 & 31 & $21-26$ & 4943.59 & $01 / 30 / 2006$ & 03/10/2009 & Vented \\
\hline $\mathrm{B} 16 \mathrm{c}$ & 350356106393903 & 51 & $41-46$ & 4943.59 & $01 / 30 / 2006$ & 03/10/2009 & Vented \\
\hline B17a & 350354106394201 & 16 & $6-11$ & 4939.60 & $01 / 27 / 2006$ & 03/10/2009 & Vented \\
\hline $\mathrm{B} 17 \mathrm{~b}$ & 350354106394202 & 31 & $21-26$ & 4939.60 & $01 / 30 / 2006$ & 03/10/2009 & Vented \\
\hline $\mathrm{B} 18 \mathrm{a}$ & 350353106394301 & 20 & $10-15$ & 4943.14 & \multicolumn{2}{|c|}{ Uninstrumented during study } & na \\
\hline BS1 & 350403106392410 & na & na & 4942.04 & $06 / 21 / 2006$ & 03/10/2009 & Vented \\
\hline BS2 & 350402106392810 & na & na & 4942.86 & $06 / 21 / 2006$ & 03/10/2009 & Vented \\
\hline BS3 & 350359106394410 & na & na & 4935.89 & $06 / 21 / 2006$ & 03/10/2009 & Vented \\
\hline R1a & 350137106395101 & 27 & $17-22$ & 4931.35 & $01 / 01 / 2004$ & $10 / 27 / 2008$ & Unvented \\
\hline R1c & 350137106395102 & 59 & $49-54$ & 4931.35 & $01 / 01 / 2004$ & $10 / 27 / 2008$ & Unvented \\
\hline \multicolumn{8}{|c|}{ Rio Bravo transects } \\
\hline $\mathrm{R} 2 \mathrm{a}$ & 350141106400701 & 16 & $6-11$ & 4927.27 & $10 / 28 / 2008$ & $03 / 12 / 2009$ & Unvented \\
\hline R3a & 350141106400801 & 17 & $7-12$ & 4927.51 & $01 / 01 / 2004$ & 03/12/2009 & Unvented \\
\hline $\mathrm{R} 3 \mathrm{c}$ & 350141106400802 & 57 & $47-52$ & 4927.51 & $01 / 01 / 2004$ & $10 / 27 / 2008$ & Unvented \\
\hline
\end{tabular}


Table 1. Site data for piezometers and surface-water data collection sites, Rio Grande inner valley, Albuquerque, New Mexico.-Continued

[S, surface-water stage gage; B, Barelas; I, I-25; M, Montaño; P, Paseo del Norte; R, Rio Bravo; a, shallow piezometer; b, mid-depth piezometer; c, deep piezometer; MP, measuring point; na, not applicable; NAVD 88, North American Vertical Datum of 1988]

\begin{tabular}{|c|c|c|c|c|c|c|c|}
\hline \multirow{2}{*}{$\begin{array}{c}\text { Other } \\
\text { identifier } \\
\text { (fig. 3a-e) }\end{array}$} & \multirow[b]{2}{*}{ Site identifier } & \multirow{2}{*}{$\begin{array}{l}\text { Piezometer depth } \\
\text { (feet below land } \\
\text { surface, rounded } \\
\text { to nearest foot) }\end{array}$} & \multirow{2}{*}{$\begin{array}{l}\text { Screened interval } \\
\text { (feet below land } \\
\text { surface, rounded to } \\
\text { nearest foot) }\end{array}$} & \multirow{2}{*}{$\begin{array}{c}\text { Land surface }^{1} \\
\text { or MP2 elevation } \\
\text { (feet above NAVD 88) }\end{array}$} & \multicolumn{2}{|c|}{$\begin{array}{c}\text { Period of record presented in this } \\
\text { report }\end{array}$} & \multirow{2}{*}{$\begin{array}{l}\text { Type of transducer in wel } \\
\text { for period of record } \\
\text { presented in this report }\end{array}$} \\
\hline & & & & & Start Date & End Date & \\
\hline \multicolumn{8}{|c|}{ Rio Bravo transects-continued } \\
\hline $\mathrm{R} 4 \mathrm{a}$ & 350138106401102 & 22 & $12-17$ & 4929.91 & $01 / 01 / 2004$ & $03 / 15 / 2009$ & Unvented \\
\hline $\mathrm{R} 4 \mathrm{~b}$ & 350138106401104 & 30 & $20-25$ & 4929.91 & $07 / 12 / 2005$ & $10 / 28 / 2008$ & Unvented \\
\hline $\mathrm{R} 4 \mathrm{c}$ & 350140106401701 & 56 & $46-51$ & 4929.91 & $01 / 01 / 2004$ & $03 / 15 / 2009$ & Unvented \\
\hline R5a & 350140106401704 & 19 & $9-14$ & 4930.49 & $07 / 12 / 2005$ & $03 / 15 / 2009$ & Unvented \\
\hline $\mathrm{R} 5 \mathrm{~b}$ & 350140106401703 & 30 & $20-25$ & 4930.49 & $07 / 12 / 2005$ & $03 / 15 / 2009$ & Unvented \\
\hline $\mathrm{R} 5 \mathrm{c}$ & 350140106401702 & 54 & $44-49$ & 4930.49 & $01 / 01 / 2004$ & $04 / 14 / 2008$ & Unvented \\
\hline R6a & 350143106402401 & 15 & $5-10$ & 4928.75 & $07 / 12 / 2005$ & $03 / 15 / 2009$ & Unvented \\
\hline R6b & 350143106402402 & 30 & $20-25$ & 4928.62 & $10 / 05 / 2005$ & $03 / 15 / 2009$ & Unvented \\
\hline $\mathrm{R} 7 \mathrm{a}$ & 350143106402503 & 15 & $5-10$ & 4928.79 & $07 / 12 / 2005$ & $03 / 15 / 2009$ & Unvented \\
\hline $\mathrm{R} 7 \mathrm{~b}$ & 350143106402501 & 35 & $25-30$ & 4928.99 & $01 / 01 / 2004$ & $08 / 15 / 2006$ & Unvented \\
\hline $\mathrm{R} 7 \mathrm{c}$ & 350143106402502 & 54 & $44-49$ & 4928.99 & $01 / 01 / 2004$ & 03/15/2009 & Unvented \\
\hline R8a & 350142106402701 & 15 & $5-10$ & 4928.32 & $01 / 01 / 2004$ & $03 / 12 / 2009$ & Unvented \\
\hline $\mathrm{R} 8 \mathrm{c}$ & 350142106402702 & 50 & $40-45$ & 4928.32 & $01 / 01 / 2004$ & 03/12/2009 & Unvented \\
\hline R9a & 350142106402801 & 14 & 4-9 & 4927.73 & $10 / 29 / 2008$ & $03 / 12 / 2009$ & Unvented \\
\hline R10a & 350137106403501 & 30 & $20-25$ & 4925.90 & $01 / 01 / 2004$ & $03 / 12 / 2009$ & Unvented \\
\hline $\mathrm{R} 10 \mathrm{c}$ & 350137106403502 & 49 & $39-44$ & 4925.90 & $01 / 01 / 2004$ & $03 / 12 / 2009$ & Unvented \\
\hline R11a & 350144106400703 & 18 & 8-13 & 4926.48 & $10 / 28 / 2008$ & $03 / 12 / 2009$ & Unvented \\
\hline R12a & 350144106400701 & 16 & $6-11$ & 4929.06 & $08 / 15 / 2006$ & $03 / 12 / 2009$ & Vented \\
\hline $\mathrm{R} 12 \mathrm{~b}$ & 350144106400702 & 31 & $21-26$ & 4929.06 & $08 / 15 / 2006$ & $03 / 12 / 2009$ & Vented \\
\hline R13a & 350144106401101 & 15 & $5-10$ & 4929.13 & $02 / 15 / 2005$ & $03 / 15 / 2009$ & Unvented \\
\hline $\mathrm{R} 13 \mathrm{~b}$ & 350144106401102 & 30 & $20-25$ & 4929.13 & $02 / 16 / 2005$ & $10 / 28 / 2008$ & Unvented \\
\hline $\mathrm{R} 13 \mathrm{c}$ & 350144106401103 & 56 & $46-51$ & 4929.13 & $02 / 16 / 2005$ & 03/15/2009 & Unvented \\
\hline R14a & 350146106401801 & 15 & $5-10$ & 4929.92 & $01 / 25 / 2005$ & 03/15/2009 & Unvented \\
\hline $\mathrm{R} 14 \mathrm{~b}$ & 350146106401802 & 30 & $20-25$ & 4929.69 & $01 / 25 / 2005$ & $03 / 15 / 2009$ & Unvented \\
\hline $\mathrm{R} 15 \mathrm{a}$ & 350147106402601 & 15 & $5-10$ & 4929.77 & $10 / 05 / 2005$ & $03 / 15 / 2009$ & Unvented \\
\hline $\mathrm{R} 15 \mathrm{~b}$ & 350147106402602 & 30 & $20-25$ & 4929.77 & $06 / 02 / 2005$ & 03/15/2009 & Unvented \\
\hline R16a & 350147106402501 & 15 & $5-10$ & 4928.70 & $10 / 05 / 2005$ & $03 / 15 / 2009$ & Unvented \\
\hline R16b & 350147106402502 & 30 & $20-25$ & 4928.70 & $06 / 02 / 2005$ & $10 / 29 / 2008$ & Unvented \\
\hline $\mathrm{R} 16 \mathrm{c}$ & 350147106402503 & 54 & $44-49$ & 4928.70 & $06 / 02 / 2005$ & $03 / 15 / 2009$ & Unvented \\
\hline
\end{tabular}




\begin{tabular}{|c|c|c|c|c|c|c|c|}
\hline \multirow{2}{*}{$\begin{array}{l}\text { Other } \\
\text { identifier } \\
\text { (fig. 3a-e) }\end{array}$} & \multirow[b]{2}{*}{ Site identifier } & \multirow{2}{*}{$\begin{array}{l}\text { Piezometer depth } \\
\text { (feet below land } \\
\text { surface, rounded } \\
\text { to nearest foot) }\end{array}$} & \multirow{2}{*}{$\begin{array}{l}\text { Screened interval } \\
\text { (feet below land } \\
\text { surface, rounded to } \\
\text { nearest foot) }\end{array}$} & \multirow{2}{*}{$\begin{array}{c}\text { Land surface }^{1} \\
\text { or MP² }^{2} \text { elevation } \\
\text { (feet above NAVD 88) }\end{array}$} & \multicolumn{2}{|c|}{$\begin{array}{c}\text { Period of record presented in this } \\
\text { report }\end{array}$} & \multirow{2}{*}{$\begin{array}{l}\text { Type of transducer in well } \\
\text { for period of record } \\
\text { presented in this report }\end{array}$} \\
\hline & & & & & Start Date & End Date & \\
\hline \multicolumn{8}{|c|}{ Rio Bravo transects - Continued } \\
\hline $\mathrm{R} 17 \mathrm{a}$ & 350147106402801 & 16 & $6-11$ & 4928.18 & $10 / 05 / 2005$ & 03/12/2009 & Unvented \\
\hline $\mathrm{R} 17 \mathrm{~b}$ & 350147106402802 & 31 & $21-26$ & 4928.18 & $10 / 05 / 2005$ & 03/12/2009 & Unvented \\
\hline $\mathrm{R} 18 \mathrm{a}$ & 350147106402701 & 15 & $5-10$ & 4929.11 & $10 / 29 / 2008$ & 03/12/2009 & Unvented \\
\hline RS1 & 350141106400810 & na & na & 4925.22 & 09/29/2006 & 03/12/2009 & Vented \\
\hline RS2 & 350145106400810 & na & na & 4926.52 & 04/10/2006 & 03/12/2009 & Vented \\
\hline RS3 & 350143106402301 & na & na & 4934.74 & $01 / 01 / 2004$ & 03/15/2009 & Unvented \\
\hline RS4 & 350142106402810 & na & na & 4927.49 & 04/07/2006 & 03/12/2009 & Vented \\
\hline RS5 & 350147106402810 & na & na & 4928.69 & $10 / 13 / 2006$ & 03/12/2009 & Vented \\
\hline \multicolumn{8}{|c|}{$\mathrm{I}-25$ transects } \\
\hline I1a & 350358106391301 & 16 & $6-11$ & 4904.92 & $11 / 29 / 2005$ & $02 / 21 / 2008$ & Unvented \\
\hline $\mathrm{I} 1 \mathrm{~b}$ & 350358106391302 & 31 & $21-26$ & 4904.92 & $06 / 01 / 2005$ & $12 / 11 / 2006$ & Unvented \\
\hline I1c & 350358106391303 & 56 & $46-51$ & 4904.92 & 06/01/2005 & $10 / 21 / 2008$ & Unvented \\
\hline $\mathrm{I} 2 \mathrm{a}$ & 345703106403901 & 16 & $6-11$ & 4902.76 & $06 / 01 / 2005$ & $10 / 21 / 2008$ & Unvented \\
\hline $\mathrm{I} 2 \mathrm{~b}$ & 345703106403902 & 31 & $21-26$ & 4902.76 & $06 / 01 / 2005$ & $10 / 21 / 2008$ & $\begin{array}{c}\text { Unvented } 06 / 01 / 2005- \\
02 / 17 / 2007), \text { vented }(03 / 21 / 2007 \\
-10 / 21 / 2008)\end{array}$ \\
\hline $13 \mathrm{a}$ & 345703106404001 & 16 & $6-11$ & 4902.35 & $11 / 29 / 2005$ & $10 / 21 / 2008$ & Unvented \\
\hline $\mathrm{I} 4 \mathrm{a}$ & 345701106404501 & 14 & $4-9$ & 4903.47 & $11 / 29 / 2005$ & $10 / 21 / 2008$ & Unvented \\
\hline $\mathrm{I} 4 \mathrm{~b}$ & 345701106404502 & 29 & $19-24$ & 4903.47 & $06 / 01 / 2005$ & $10 / 21 / 2008$ & Unvented \\
\hline $\mathrm{I} 4 \mathrm{c}$ & 345701106404503 & 54 & $44-49$ & 4903.47 & 06/01/2005 & $12 / 11 / 2006$ & Unvented \\
\hline $15 \mathrm{a}$ & 345701106404601 & 14 & 4-9 & 4904.60 & $11 / 29 / 2005$ & $10 / 21 / 2008$ & Unvented \\
\hline $\mathrm{I} 5 \mathrm{~b}$ & 345701106404602 & 29 & $19-24$ & 4904.60 & 06/01/2005 & $10 / 21 / 2008$ & Unvented \\
\hline I6a & 345707106410101 & 14 & 4-9 & 4905.91 & $11 / 30 / 2005$ & $10 / 24 / 2008$ & Unvented \\
\hline I6b & 345707106410102 & 29 & $19-24$ & 4905.91 & $06 / 01 / 2005$ & $11 / 19 / 2007$ & $\begin{array}{c}\text { Unvented }(06 / 01 / 2005 \text { - } \\
06 / 28 / 2006) \text {, vented }(06 / 28 / 2006 \\
-11 / 19 / 2007)\end{array}$ \\
\hline $17 \mathrm{a}$ & 345706106410201 & 14 & $4-9$ & 4905.12 & $11 / 29 / 2005$ & $10 / 24 / 2008$ & Unvented \\
\hline $\mathrm{I} 7 \mathrm{~b}$ & 345706106410202 & 29 & $19-24$ & 4905.12 & $06 / 01 / 2005$ & $10 / 24 / 2008$ & Unvented \\
\hline $\mathrm{I} 7 \mathrm{c}$ & 345706106410203 & 49 & $39-44$ & 4905.12 & $04 / 22 / 2005$ & $10 / 24 / 2008$ & Unvented \\
\hline I8a & 345704106410701 & 15 & $5-10$ & 4901.90 & $11 / 29 / 2005$ & $10 / 24 / 2008$ & Unvented \\
\hline $\mathrm{I} 8 \mathrm{~b}$ & 345704106410702 & 30 & $20-25$ & 4901.90 & $06 / 01 / 2005$ & $10 / 24 / 2008$ & Unvented \\
\hline I10a & 345703106411201 & 13 & 3-8 & 4900.97 & $06 / 01 / 2005$ & $10 / 22 / 2008$ & Unvented \\
\hline
\end{tabular}


Table 1. Site data for piezometers and surface-water data collection sites, Rio Grande inner valley, Albuquerque, New Mexico.-Continued

[S, surface-water stage gage; B, Barelas; I, I-25; M, Montaño; P, Paseo del Norte; R, Rio Bravo; a, shallow piezometer; b, mid-depth piezometer; c, deep piezometer; MP, measuring point; na, not applicable; NAVD 88, North American Vertical Datum of 1988]

\begin{tabular}{|c|c|c|c|c|c|c|c|}
\hline \multirow{2}{*}{$\begin{array}{l}\text { Other } \\
\text { identifier } \\
\text { (fig. 3a-e) }\end{array}$} & \multirow[b]{2}{*}{ Site identifier } & \multirow{2}{*}{$\begin{array}{l}\text { Piezometer depth } \\
\text { (feet below land } \\
\text { surface, rounded } \\
\text { to nearest foot) }\end{array}$} & \multirow{2}{*}{$\begin{array}{l}\text { Screened interval } \\
\text { (feet below land } \\
\text { surface, rounded to } \\
\text { nearest foot) }\end{array}$} & \multirow{2}{*}{$\begin{array}{c}\text { Land surface }^{1} \\
\text { or MP² elevation } \\
\text { (feet above NAVD 88) }\end{array}$} & \multicolumn{2}{|c|}{$\begin{array}{c}\text { Period of record presented in this } \\
\text { report }\end{array}$} & \multirow{2}{*}{$\begin{array}{l}\text { Type of transducer in well } \\
\text { for period of record } \\
\text { presented in this report }\end{array}$} \\
\hline & & & & & Start Date & End Date & \\
\hline \multicolumn{8}{|c|}{ I-25 transects-Continued } \\
\hline $\mathrm{I} 10 \mathrm{~b}$ & 345703106411202 & 28 & $18-23$ & 4900.97 & $06 / 01 / 2005$ & $11 / 05 / 2007$ & Unvented \\
\hline I10c & 345703106411203 & 48 & $38-43$ & 4900.97 & 06/01/2005 & $10 / 23 / 2008$ & Unvented \\
\hline I11a & 345707106404101 & 13 & $3-8$ & 4902.27 & $02 / 23 / 2007$ & $10 / 20 / 2008$ & Vented \\
\hline $\mathrm{I} 11 \mathrm{~b}$ & 345707106404102 & 28 & $18-23$ & 4902.27 & $01 / 20 / 2006$ & $10 / 20 / 2008$ & Vented \\
\hline $\mathrm{I} 12 \mathrm{a}$ & 345707106404103 & 13 & $3-8$ & 4902.28 & \multicolumn{2}{|c|}{ Uninstrumented during study } & na \\
\hline I13a & 345706106404701 & 14 & $4-9$ & 4904.21 & 03/09/2007 & $10 / 21 / 2008$ & Vented \\
\hline $\mathrm{I} 13 \mathrm{~b}$ & 345706106404702 & 29 & $19-24$ & 4904.21 & $12 / 12 / 2006$ & $11 / 06 / 2007$ & Unvented \\
\hline $\mathrm{I} 13 \mathrm{c}$ & 345706106404703 & 49 & $34-39$ & 4904.21 & $12 / 12 / 2006$ & $10 / 21 / 2008$ & Vented \\
\hline $\mathrm{I} 14 \mathrm{a}$ & 345706106404704 & 14 & 4-9 & 4904.06 & 03/09/2007 & $10 / 21 / 2008$ & Vented \\
\hline $\mathrm{I} 14 \mathrm{~b}$ & 345706106404705 & 29 & $19-24$ & 4904.06 & $01 / 20 / 2006$ & $10 / 21 / 2008$ & Unvented \\
\hline $\mathrm{I} 15 \mathrm{a}$ & 345713106410604 & 14 & 4-9 & 4906.50 & $10 / 13 / 2006$ & $10 / 27 / 2008$ & Vented \\
\hline $\mathrm{I} 15 \mathrm{~b}$ & 345713106410605 & 29 & $19-24$ & 4906.50 & $10 / 13 / 2006$ & $10 / 27 / 2008$ & Unvented \\
\hline I16a & 345713106410601 & 16 & $6-11$ & 4908.22 & \multicolumn{2}{|c|}{ Uninstrumented during study } & na \\
\hline $\mathrm{I} 16 \mathrm{~b}$ & 345713106410602 & 31 & $21-26$ & 4908.22 & $01 / 23 / 2006$ & $10 / 27 / 2008$ & Vented \\
\hline I16c & 345713106410603 & 49 & $39-44$ & 4908.22 & $01 / 23 / 2006$ & $10 / 27 / 2008$ & Vented \\
\hline $\mathrm{I} 17 \mathrm{a}$ & 345713106411001 & 13 & $3-8$ & 4903.46 & 03/09/2007 & $10 / 23 / 2008$ & Vented \\
\hline $\mathrm{I} 17 \mathrm{~b}$ & 345713106411002 & 28 & $18-23$ & 4903.46 & $01 / 23 / 2006$ & $10 / 23 / 2008$ & Vented \\
\hline IS1 & 345703106404010 & na & na & 4903.63 & $04 / 07 / 2006$ & $10 / 21 / 2008$ & Vented \\
\hline IS2 & 345705106405810 & na & na & $4905.14^{3} 4906.66^{4}$ & $08 / 10 / 2005$ & $10 / 23 / 2008$ & $\begin{array}{c}\text { Unvented }(08 / 10 / 2005- \\
06 / 28 / 2006) \text {, vented }(07 / 06 / 2006 \\
-10 / 23 / 2008)\end{array}$ \\
\hline IS3 & 345705106410810 & na & na & $4902.04^{5} 4903.23^{6}$ & 04/07/2006 & $10 / 23 / 2008$ & Vented \\
\hline
\end{tabular}

${ }^{1}$ Land-surface elevation for piezometers only

${ }^{2}$ Measuring-point elevation for surface-water stage gages only

${ }^{3}$ MP valid through May 2, 2006

${ }^{4} \mathrm{MP}$ valid after May 2, 2006

${ }^{5}$ MP valid through May 8, 2008

${ }^{6} \mathrm{MP}$ valid after May 8, 2008 
Groundwater-level data were measured using vented and unvented transducers (table 1). Transducers with vented cable are automatically compensated for changes in barometric pressure, but transducers with unvented cable are not. With unvented transducers, a calculation is required to correct the recorded water levels for changes in barometric pressure. Unvented transducers record total pressure (water + air) and convert this value to a water level. Any change in air pressure needs to be subtracted from an initial barometric pressure (pressure at the start of the water level data set being corrected) and the result subtracted from the recorded total water level in order to attain a corrected water level (Freeman and others, 2004). Hourly barometric pressure values from the Albuquerque airport (National Climatologic Data Center, 2011) were used to correct all water level data collected using unvented transducers. Water-level barometric corrections ranged from 0 to about $0.5 \mathrm{ft}$.

\section{Slug Tests}

The spatial variability of aquifer properties at each of the transect locations was estimated by conducting slug tests during January and February 2009. Analytical results from slug tests, a type of aquifer test, need to be interpreted carefully because the continuity and distribution of hydrologically distinguishable lithologies are unique to different parts of the alluvial aquifer. Type-curve matching methods are the most common analytical techniques used for slug-test analysis and provide an estimate of aquifer characteristics in different portions of the aquifer.

Slug tests were conducted by the rapid introduction of a 60-inch long weighted PVC slug, with a diameter of 0.75 inch, into a 1 -inch diameter piezometer to induce a positive displacement of water in the piezometer of $1 \mathrm{ft}$ or more. Water levels were recorded prior to and during the slug tests to record initial head, slug insertion, and water-level recovery to static conditions. After water levels recovered to within 5 percent of initial water levels (Butler, 1998), the slug was removed and the subsequent water-level recovery was recorded at 1-second intervals by using pressure transducers. Slug tests were performed at 15 shallow piezometers, 19 middepth piezometers, and 1 deep piezometer at the Paseo Del Norte, Montaño, Barelas, Rio Bravo, and I-25 transects.

Hydraulic-conductivity estimates were determined by the Bouwer and Rice (1976) and Butler (1998) methods for slug-test analysis in unconfined aquifers. The Butler (1998) method is an extension of the Bouwer and Rice (1976) method to account for slug tests conducted in highly permeable aquifers with an oscillatory response during recovery. For both methods, the recovery data are analyzed by using a family of type curves to determine the hydraulic conductivity of the aquifer. The Bouwer and Rice (1976) method is used to estimate hydraulic conductivity through the following equation:

$$
K=\frac{r_{c}^{2} \ln \left(R_{e} / r_{w}\right)}{2 L} \frac{1}{t} \ln \frac{y_{0}}{y_{t}}
$$

where

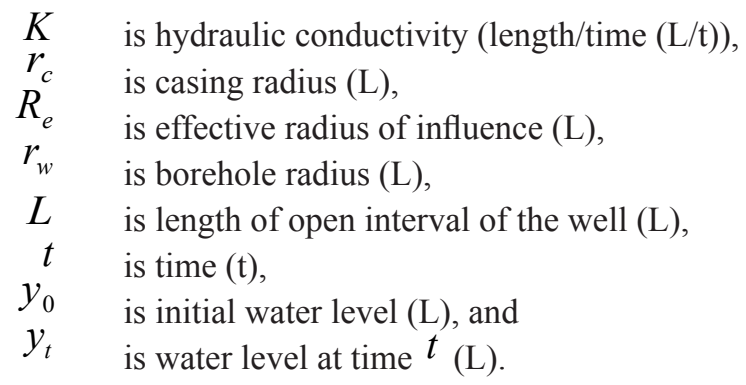

The Butler (1998) method for an unconfined, highly permeable aquifer is used to estimate hydraulic conductivity through the following equation:

$$
K_{r}=\frac{t_{d}^{*}}{t^{*}} \frac{r_{c}^{2} \ln \left(R_{e} / r_{w}\right)}{2 b_{s} C_{D}^{*}}
$$

where

$$
\begin{array}{cl}
K_{r} & \text { is radial hydraulic conductivity }(\mathrm{L} / \mathrm{t}), \\
t_{d}{ }^{*} & \text { is dimensionless time parameter }\left(\frac{g}{L_{e}}\right)^{1 / 2} t, \\
& \text { where } g \text { is gravitational acceleration, and } \\
L_{e} \text { is effective length of water column } & \text { in well, and t is time. } \\
t^{*} & \text { is time (t), } \\
r_{c} & \text { is casing radius (L), } \\
R_{e} & \text { is effective radius of influence (L), } \\
r_{w} & \text { is borehole radius (L), } \\
b_{s} & \text { is screen length (L), and } \\
C_{D}^{*} & \text { is dimensionless damping parameter. }
\end{array}
$$

Use of either method assumes (1) the aquifer is unconfined and infinite in areal extent; (2) the aquifer is homogeneous, isotropic, and uniform in thickness; (3) the water table is initially horizontal; (4) the well is fully or partially penetrating; (5) the water level in the well is stable; (6) the slug is introduced rapidly into the well; and (7) the screened interval is completely saturated during testing.

\section{Darcy's Law}

Temporal changes in the rate of Rio Grande seepage to the alluvial aquifer were computed by using Darcy's law. Darcy flux, or specific discharge, is a rate of groundwater movement defined by a hydraulic gradient acting across a 
homogeneous aquifer under laminar flow conditions

(Fetter, 1994). In equation form, Darcy's law is:

$$
Q=-K A \frac{d h}{d l}
$$

where

$$
\begin{aligned}
& Q \quad \text { is volumetric groundwater flow }\left(\mathrm{L}^{3} / \mathrm{t}\right), \\
& K \quad \text { is hydraulic conductivity }(\mathrm{L} / \mathrm{t}), \\
& \text { is cross-sectional area through which } \\
& \text { groundwater flow occurs }\left(\mathrm{L}^{2}\right) \text {, and } \\
& \text { is hydraulic gradient (dimensionless but, by } \\
& \text { convention, is negative in the direction of } \\
& \text { groundwater flow). }
\end{aligned}
$$

Dividing both sides by the cross-sectional area $(A)$, and setting $q=Q / A$, the Darcy flux through a unit area of aquifer can be stated as:

$$
q=-K \frac{d h}{d l}
$$

where

$$
\begin{aligned}
& q \text { is the Darcy flux of water through the } \\
& \text { aquifer }(\mathrm{L} / \mathrm{t}) \text {. }
\end{aligned}
$$

The hydraulic conductivity values used in the Darcy flux calculations in this report are considered bulk values that characterize the aquifer as a homogeneous mixture of sediments. Bulk hydraulic conductivity values represent average conditions in the aquifer and were defined on the basis of the results of slug tests conducted for this study and on the basis of values obtained from published literature (Tiedeman and others, 1998; Bartolino and Niswonger, 1999; McAda, 2001; Sanford and others, 2003; Moret, 2007).

Horizontal hydraulic gradients used in the Darcy flux calculations were calculated by using the three-point method to determine the strike (compass direction) and dip (slope) of rock outcrops (Compton, 1962), and which has been used by Myers and others (1999) to calculate hydraulic gradients in an alluvial aquifer at Fort Riley, Kansas. Daily hydraulic gradients were calculated by using the three-point method and 2006-08 daily mean water levels to compute the slope of the water surface between selected sets of shallow piezometers. Daily mean water levels were not uniformly available at all transects from 2006-08 (table 1). For each of the paired transects, hydraulic gradients were calculated for three-piezometer combinations of adjacent, triangle-forming piezometers (piezometer triangles) between the river and the riverside drain on each side of the Rio Grande (fig. 4). For each set of six piezometers, a total of eight piezometer triangles were defined on each side of the river (fig. 4). After the daily mean hydraulic gradient was calculated for each of the eight piezometer triangles, the daily mean hydraulic gradient for each side of the river was calculated as the average of the daily mean hydraulic gradients of the eight piezometer triangles.

\section{Suzuki-Stallman Method}

In addition to specific fluxes calculated from hydraulic conductivities and hydraulic gradients by using the Darcy equation, specific fluxes also were computed with the temperature data collected at 10 - and $20-\mathrm{ft}$ depths in the alluvial aquifer by using the analytical method developed by Suzuki (1960) and Stallman (1965) that was applied to horizontal flux by Moret (2007). Suzuki (1960) developed an equation and approximate analytical solution for 1-dimensional (vertical) advective and conductive heat transport into rice paddy soils assuming saturated, vertical, steady-state flow in a homogenous medium with a sinusoidal daily surface temperature. Later, on the basis of Suzuki's work, Stallman (1965) developed an exact analytic solution for 1-dimensional (vertical) advective and conductive heat transfer in an aquifer. The Suzuki-Stallman method relies only on temperature data, providing an independent method that

\begin{tabular}{|c|c|}
\hline$k$ & $\begin{array}{l}\text { is the thermal conductivity of the saturated } \\
\text { aquifer sediment and water) (BTU } / \mathrm{B}^{\circ} / \mathrm{L}^{\circ} \mathrm{F} \text {, }\end{array}$ \\
\hline$T$ & is the temperature in the aquifer $\left({ }^{\circ} \mathrm{F}\right)$, \\
\hline$\rho_{w} c_{w}$ & $\begin{array}{l}\text { is the heat capacity of the water }\left(\mathrm{BTU}_{\mathrm{IT}} / \mathrm{L}^{3 \circ} \mathrm{F}\right) \\
\text { [British Thermal Unit (International } \\
\text { Steam Table Calorie) per cubic foot- } \\
\text { degrees Fahrenheit, }\end{array}$ \\
\hline$q_{s}$ & $\begin{array}{l}\text { is the specific flux of water through the } \\
\text { aquifer }(\mathrm{L} / \mathrm{t}) \text {, }\end{array}$ \\
\hline$\rho c$ & $\begin{array}{l}\text { is the heat capacity of the saturated aquifer } \\
\text { (sediment and water) }\left(\mathrm{BTU}_{\mathrm{IT}} / \mathrm{L}^{3 \circ} \mathrm{F}\right) \text {, }\end{array}$ \\
\hline$z$ & is distance $(\mathrm{L})$, and \\
\hline$t$ & is time $(\mathrm{t})$. \\
\hline
\end{tabular}
can be used to check the Darcy flux estimates generated by using measured hydraulic conductivity values and hydraulic gradients. For a more complete discussion of using heat as a groundwater tracer see Anderson (2005), Blasch and others (2007), or Constantz and others (2008).

The equation describing one-dimensional, vertical transport is (Suzuki, 1960):

$$
k \frac{\partial^{2} T}{\partial z^{2}}+\rho_{w} c_{w} q_{s} \frac{\partial T}{\partial z}=\rho c \frac{\partial T}{\partial t}
$$

where 

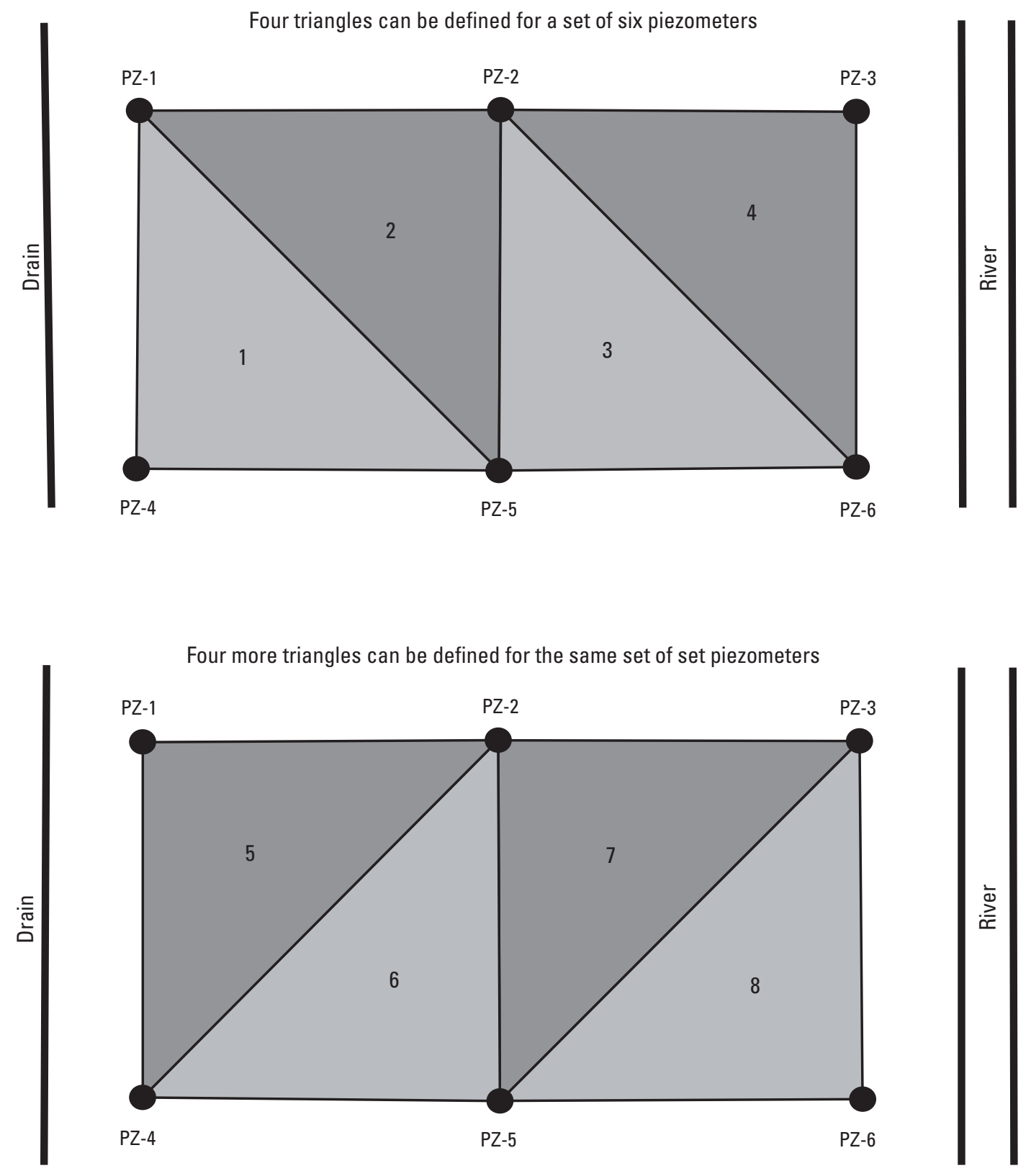

Figure 4. Diagram of sets of piezometers used to compute daily mean hydraulic gradients on each side of the Rio Grande for each paired transect. For six piezometers, eight triangles can be defined for use in gradient calculations. 
Moret (2007) adapted the heat transport equation (Suzuki, 1960) and analytical solution (Stallman, 1965) to use annual temperature variations in river temperature and their propagation into the adjacent aquifer to estimate rates of horizontal river seepage. For the horizontal flux, Moret (2007) uses Equation 5, but substitutes horizontal distance $\mathrm{x}$ for the vertical distance $\mathrm{z}$. Temperature variations in the river affect the solution to equation 5; consequently Moret (2007) adapted Stallman's (1965) method for incorporating diurnal heating and cooling of the land surface to correct for changes in river temperature. To use the solution proposed by Stallman, Moret found it necessary to assume that the aquifer is bounded at $x=0$ by a fully penetrating river and that all flow is uniform and horizontal in the positive $\mathrm{x}$ direction. Following Suzuki (1960) and Stallman (1965), Moret (2007) stated that the temperature of the river, $T_{\text {river }}$, varies sinusoidally with a period of 1 year:

$$
T_{\text {river }}=T_{\text {avg }}+T_{0} \sin \left(\frac{2 \pi}{\tau} t+\varphi\right)
$$

where

$$
\begin{aligned}
& T_{\text {avg }} \quad \text { is the average river temperature }\left({ }^{\circ} \mathrm{F}\right) \text {, } \\
& T_{0} \quad \text { is the magnitude of temperature oscillation in } \\
& \text { the river }\left({ }^{\circ} \mathrm{F}\right) \text {, } \\
& \tau \quad \text { is the period of the oscillation ( } t \text { ), } \\
& \varphi \quad \text { is the phase lag in temperature signal of the } \\
& \text { river }(\mathrm{t}) \text {, and } \\
& t \text { is time (t). }
\end{aligned}
$$

If the temperature dependence of the viscosity of water is ignored, the analytical solution for advective and conductive heat transport proposed by Suzuki (1960) and further developed by Stallman (1965) can be used to model the temperature oscillations in groundwater $\left(\mathrm{T}_{\text {osc }}\right)$ that are attributable to river water temperature oscillations $\left(T_{\text {river }}\right)$ :

$$
T_{\text {osc }}=T_{0} e^{-a x} \sin \left(\frac{2 \pi}{\tau} t-b x+\varphi\right)
$$

where

$$
\begin{gathered}
T_{0}, \tau, t, x, \text { and } \varphi \text { are as previously defined for } \\
\text { equations } 5 \text { and } 6,
\end{gathered}
$$

$$
a=\left[\sqrt{K_{T}^{2}+\frac{V^{4}}{4}}+\frac{V^{2}}{2}\right]^{\frac{1}{2}}-V \text {, and }
$$

$$
b=\left[\sqrt{K_{T}^{2}+\frac{V^{4}}{4}}-\frac{V^{2}}{2}\right]^{\frac{1}{2}}
$$

The parameter $a$, with units of $\mathrm{ft}^{-1}$, controls the attenuation of the temperature wave. The spatial frequency $b$, with units of radians per foot $(\mathrm{rad} / \mathrm{ft})$, controls the propagation of the wave through space. The parameters $K_{T}$ and $V$ are defined by:

$$
\begin{gathered}
K_{T}=\frac{\pi \rho c}{k \tau} \\
V=\frac{\rho_{w} c_{w} q_{s}}{2 k}
\end{gathered}
$$

where $\rho c, k, \tau, \rho_{w} c_{w}$, and $q_{s}$ are as previously defined in equations 5 and 6 .

Figure 5 shows how theoretical values of $a$ and $b$, calculated through use of equations 8 and 9 , vary as a function of changes in the flux term $\left(q_{\mathrm{s}}\right)$ in equation 11. Type curves in figure 5 were constructed by using the average of the four values obtained by Bartolino and Niswonger (1999) for the thermal conductivity $\left(1.25 \mathrm{BTU}_{\mathrm{IT}} / \mathrm{hr} \mathrm{ft}{ }^{\circ} \mathrm{F}\right)$, and saturated heat capacity $\left(155 \mathrm{BTU}_{\mathrm{IT}} / \mathrm{ft}^{3}{ }^{\circ} \mathrm{F}\right)$ of the inner valley alluvial aquifer.

For $a$ and $b$ determined from observations, the specific flux through the aquifer, $q_{\mathrm{s}}$, can be estimated from type curves such as those in figure 5. The parameters $a$ and $b$ can be estimated by using temperature records from two wells at distances $x_{1}$ and $x_{2}$ from the river. The differences between the maximum and minimum temperatures in these records, $\Delta T x_{1}$ and $\Delta T x_{2}$, can be used to estimate $a$ :

$$
a=\frac{-1}{x_{2}-x_{1}} \log \left(\frac{\Delta T x_{1}}{\Delta T x_{2}}\right)
$$

The phase lag between the two temperature records, $\Delta t$, yields $b$ :

$$
b=\frac{2 \pi \Delta t}{\left(x_{2}-x_{1}\right) \tau}
$$

Moret (2007) found that the best way to estimate specific flux, $q_{\mathrm{s}}$, is to plot the measured values of $a$ and $b$ on type curves such as figure 5 . With this method, the user can determine whether $a$ and $b$ are physically realistic and consistent with each other and assess the sensitivity of $q_{s}$ to 


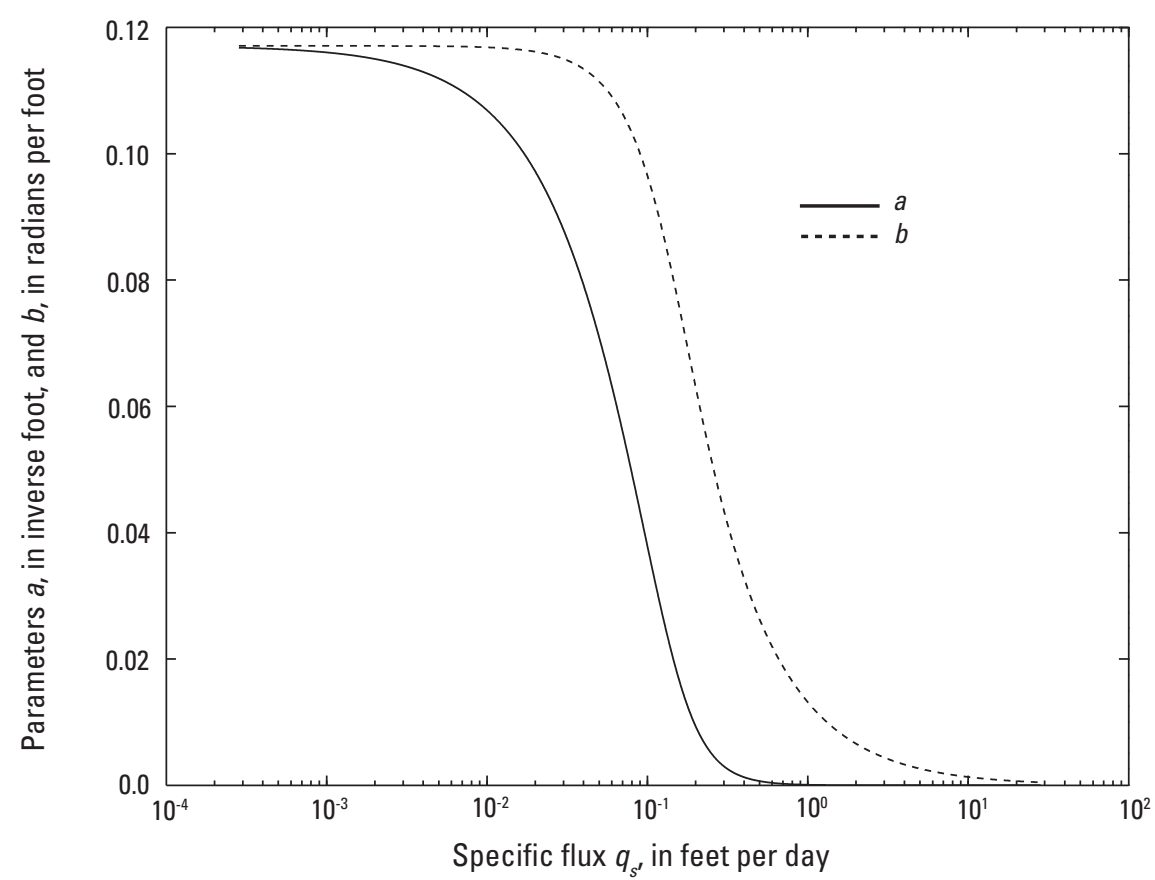

Figure 5. Type curves for the Suzuki-Stallman method, showing values of the parameters $a$ and $b$ as a function of specific flux. Type curves generated using equations $8,9,10$, and 11 .

measurement error. The Suzuki-Stallman method can only be used to estimate flux in regions of the curves where $a$ and $b$ vary as a function of $q_{s}$ (fig. 5).

In this study, there were four steps used to estimate flux with temperature data. First, two type curves ( $a$ and $b$ ) were developed by using thermal properties of the aquifer and equations 8-11 for a range of specific flux values (fig. 5). Second, values for $a$ and $b$ were calculated using equations 12 and 13 and temperatures measured in the piezometers. Third, the values of $a$ and $b$ calculated in the previous step were plotted on their respective type curves and matched with the corresponding flux $\left(q_{\mathrm{a}}\right.$ and $\left.q_{\mathrm{b}}\right)$. Fourth, the $q_{\mathrm{a}}$ and $q_{\mathrm{b}}$ estimates were averaged to obtain $q_{\text {heat }}$.

\section{Limitations and Assumptions}

According to Moret (2007), the Suzuki-Stallman method used in this study does not fully represent all of the factors that contribute to aquifer temperature signals, but in many cases it may represent the system sufficiently well to produce a useful estimate of groundwater flux. Some of the limitations and assumptions with this method are described in this section. A complete discussion of data limitations associated with use of the Suzuki-Stallman method is found in Moret (2007).

\section{Range of Applicability}

The Suzuki-Stallman method can only be used to estimate flux in regions of the curve where $a$ and $b$ vary detectably as a function of specific flux $\left(q_{s}\right)$. Figure 5 shows how the values of $a$ and $b$ vary as a function of specific flux by using average thermal properties of the aquifer that were determined by Bartolino and Niswonger (1999). The exact limits of detectability depend on the accuracy of the temperature data, the magnitude of the original temperature signal oscillation, and the distance between measuring points, but for the aquifer parameters used in this study, fluxes between $10^{-3}$ and $1 \mathrm{ft} / \mathrm{d}$ should be detectable.

\section{Flow Direction}

The heat-transport equation (Suzuki, 1960) was developed to measure vertical infiltration and assumes that the flux is one-dimensional and calculated along the flowpath. Seepage of water from the Rio Grande into the adjacent aquifer is not one-dimensional. The hydraulic gradients are 
primarily horizontal, but vertical gradients do exist. The paired piezometer transects in this study were not oriented directly along the flow-paths of river seepage and $q_{\text {heat }}$ was calculated between pairs of wells along each individual transect. Based on these limitations, the $q_{\text {heat }}$ calculations likely systematically underestimate the flux.

\section{Aquifer Heterogeneity}

The Suzuki equation (1960) assumes a uniform flow field, a condition not met in a heterogeneous aquifer. If temperature signals measured at points with different flow rates are compared, then the estimated fluxes will be incorrect. Moret (2007) advised measuring the temperature at a number of depths in as many observation wells as are available to limit the effect of time series recorded in nonrepresentative locations.

\section{Variable Recharge}

Bartolino and Niswonger (1999) determined that the flux through the riverbed at the Paseo Del Norte site varied by roughly an order of magnitude over the course of the year, but the Suzuki (1960) equation assumes a constant flux. Moret (2007) modeled aquifer temperature by using both variable monthly recharge estimates and a constant annual flux. The two models agreed reasonably well, and Moret (2007) concluded that the Suzuki-Stallman method represents aquifers with annual variations in river recharge reasonably well.

\section{Spatial Aliasing}

The spatial wavelength, $\lambda$, of a temperature wave described by equation 7 is defined by:

$$
\lambda=\frac{2 \pi}{b}
$$

As shown on figure 5 , fluxes of $10^{-3}$ and $1 \mathrm{ft} / \mathrm{d}$ result from $b$ values of 0.117 and $0.0132 \mathrm{rad} / \mathrm{ft}$, respectively, and wavelengths of about 54 and $476 \mathrm{ft}$, respectively. For example, if $q=10^{-3} \mathrm{ft} / \mathrm{d}$, then $b=0.117$ (from fig. 5) and $\lambda=2 \pi / 0.117$, or $54 \mathrm{ft}$; if $q=1$, then $b=0.0132$ (from fig. 5), and $\lambda=2 \pi / 0.0132$, or $476 \mathrm{ft}$. When the wells used to measure temperature are separated by more than one wavelength, the apparent lag between the temperature series will be incorrect. This phenomenon is known as "spatial aliasing." In this situation, $b$ will be greatly underestimated. A measured value of $b$ can be evaluated by plotting it along with the measured $a$ value for the same time series pair on a plot generated by using equation 9 (for example, fig. 5). If the $q$ values that correspond to $a$ and $b$ do not agree reasonably well, then 1-year increments can be added to the measured lag.

\section{Surface Temperature Variations}

Temperatures in shallow parts of the alluvial aquifer can be affected by the annual variation in the ground-surface temperature. The ground-surface temperature generally is an attenuated version of the atmospheric-temperature signal (Smerdon and others, 2004). Because of the exponential decay of ground-surface temperature signals with depth (Carslaw and Jaegar, 1959), groundwater temperatures measured more than approximately $5 \mathrm{ft}$ below ground surface may not be substantially affected by surface warming (Moret, 2007; Silliman and Booth, 1993).

At the Paseo del Norte site, Bartolino (2003) recorded temperatures at 7 or 10,13 or $15,20,26$, and $33 \mathrm{ft}$ below ground surface in eight piezometers installed in an east-west configuration between the riverside drains east and west of the river. In piezometers P06 and P07 from Bartolino's (2003) dataset, Moret (2007) found no substantial difference between the temperatures recorded at depths of 7 or $10 \mathrm{ft}$ and the temperatures recorded at 13 or $15 \mathrm{ft}$, indicating that the effect of atmospheric temperature variations is small. There was a $6.5^{\circ} \mathrm{F}$ difference in the magnitudes of the temperature waves recorded at 7 and $15 \mathrm{ft}$ in piezometer P08 (Bartolino, 2003). This difference, however, is greater than what would be expected if it was attributable to the atmospheric-temperature effect and is likely due to lower hydraulic conductivity of the sediments at the $7 \mathrm{ft}$ depth than the deeper sediments (Moret, 2007).

\section{Temperature Dependence of Hydraulic Conductivity}

Hydraulic conductivity depends inversely on fluid viscosity and depends directly on fluid density, whereas both viscosity and density depend on temperature. The viscosity of liquid water at $32{ }^{\circ} \mathrm{F}$ is twice that of water at 77 ${ }^{\circ} \mathrm{F}$ (Vennard and Street, 1982). Surface-water recharge fluxes can vary substantially with the temperature of the water (Constantz and others, 1994). A fundamental limitation of the Suzuki-Stallman method is that it does not consider the effect of temperature on hydraulic conductivity. Moret (2007) evaluated this limitation by using a two-dimensional finiteelement model and concluded that if temperature time-series data appear to be sinusoidal, then the temperature dependence of hydraulic conductivity does not limit the applicability of the Suzuki-Stallman method. For additional details see Moret (2007).

\section{Uncertainty in Thermal Properties}

The thermal properties of the aquifer, $\mathrm{k}$ and $\rho c$, are generally not well known, but for aquifers consisting of unconsolidated sediments, the range of $\mathrm{k}$ values reported in the literature is not large (Moret 2007). Moret (2007) found that if site-specific measurements are not readily available, a thermal conductivity chosen based solely on the aquifer's lithology will introduce an uncertainty into the estimate of $q_{s}$ of a few tens of percent. In this study, we used the average of 
the four values obtained by Bartolino and Niswonger (1999) for the thermal conductivity $\left(2.16 \mathrm{~W} \mathrm{~m}^{-1}{ }^{\circ} \mathrm{C}^{-1}\right)$, and saturated heat capacity $\left(3.2 \times 10^{6} \mathrm{~J}^{\circ} \mathrm{C}^{-1} \mathrm{~m}^{-3}\right)$ of the upper Santa Fe Group aquifer.

\section{Data Error}

Another possible limitation in the interpretation of temperature oscillations using the Suzuki-Stallman method is uncertainty in the estimation of best fit sinusoids for the observed data series, which would result in uncertainty in $a$ and $b$. Errors in sinusoid fitting are likely to be greatest closer to the source of the surface-water recharge (Moret, 2007).

\section{Groundwater Hydrology}

The hydrologic characteristics of groundwater movement in the study area were examined using slug-test, hydraulichead, and water-temperature data. Slug-test data from 35 sites were used to determine the hydraulic conductivity of the alluvial aquifer (slug-test archive, files of USGS New Mexico Water Science Center, Albuquerque). Daily mean hydraulichead data were used to establish groundwater gradients at transect locations, and vertical water-temperature profile data were collected at selected locations to evaluate the depth of penetration of river-water recharge into the aquifer. Finally, a simple conceptual model of groundwater flow away from the river was constructed to demonstrate water-table conditions and characteristics.

\section{Hydraulic Conductivity}

During slug tests, the water-level responses in 16 piezometers were nonoscillatory and were analyzed using the Bouwer and Rice (1976) slug-test analysis method. The water-level responses at 19 piezometers exhibited oscillatory behavior and were analyzed using the Butler (1998) slugtest analysis method. Results from 35 slug tests performed in the alluvial aquifer for this study indicate that the spatial variability of hydraulic properties in the alluvial aquifer is small (fig. 6). Hydraulic-conductivity values ranged from $5 \mathrm{ft} / \mathrm{d}$ (I-25 transects) to $160 \mathrm{ft} / \mathrm{d}$ (Montaño transects) (table 2). The median hydraulic-conductivity value for all transects was $40 \mathrm{ft} / \mathrm{d}$. Slug-test results from piezometers on the east side of the river were not substantially different from those on the west side.

Slug tests performed for this study hydraulically stressed only a limited portion of the aquifer surrounding each piezometer; however, the range of estimated hydraulic conductivities used in previous investigations (10 to $150 \mathrm{ft} / \mathrm{d}$ [Kernodle and others, 1995; Tiedeman and others, 1998; and McAda, 2001]) was similar to the range of median hydraulicconductivity values estimated in this study. Lithologic variability, attributable to complexly interfingered gravels, sands, silts, and clays in the alluvial aquifer, was noted in cores to occur over distances of a few tens of feet or less. Because coring was performed, in part, so the screened interval of each piezometer was placed in sandy material to ensure communication with the aquifer, the slug-test results provide a measurement of the heterogeneity of sandy zone hydraulic conductivities within the alluvial aquifer but do not provide an overall measurement of the heterogeneity of hydraulic conductivity in the aquifer. Literature-cited values determined from regional-scale modeling (Sanford and others, 2003), aquifer tests (McAda, 2001), and wellto-well heat-transport modeling (Moret, 2007) provide a basis to extrapolate the heterogeneities to the scale of the current study.

\section{Groundwater Levels and Temperatures}

Hourly groundwater-level data were recorded from 164 piezometers screened at different intervals within the alluvial aquifer. Daily mean water levels are shown in figure 7.

Groundwater-level data were used to evaluate water-level trends, measure response to increases or decreases in river stage, and calculate horizontal hydraulic gradients. In the study area, diurnal and seasonal fluctuations in water levels were common, but no substantial upward or downward long-term water-level trends were discernible from 2004 through early 2009 (fig. 7). Groundwater-level responses to stage changes in the river were measurable; water levels in piezometers closest to the river showed a more pronounced response to change in river stage than did piezometers next to or outside the drains. Similar heads typically were measured in the shallow and mid-depth piezometers; however, there were exceptions, most notably at piezometers B16a, b, and c and R4a, b, and c, where clay-rich sediments were observed in sediment cores. Horizontal hydraulic gradients indicated that groundwater movement generally was away from the river and toward the drains (fig. 3, table 3). This direction of groundwater movement makes sense because the riverside drains were designed to extend below the groundwater table, except near the tail ends of the drains where they have to empty back into the river. While drain water levels generally were lower than adjacent groundwater levels, there were instances where the water level in a drain was substantially higher than adjacent groundwater levels. For example, at the Montaño transects, the water level at MS3, near the downstream end of the Corrales Riverside Drain, was more than $5 \mathrm{ft}$ higher than the water levels at M9a and M18a on February 7, 2008 (fig. 3b). The depth of water in the drain at this location was about 3 to $4 \mathrm{ft}$, so the bottom of the drain probably was also higher than the water table. These data indicate that the hydraulic interaction between the drain and groundwater at this location is minimal; this condition is likely to persist from one year to the next. In February, 2009, discharge was measured along this portion of the Corrales Riverside Drain as part of the seepage investigation. Measurements made between drain miles 4 and 6 (fig. 1) of 3.0 and $2.6 \mathrm{ft}^{3} / \mathrm{s}$, respectively, demonstrated that the drain was losing water to the aquifer. 


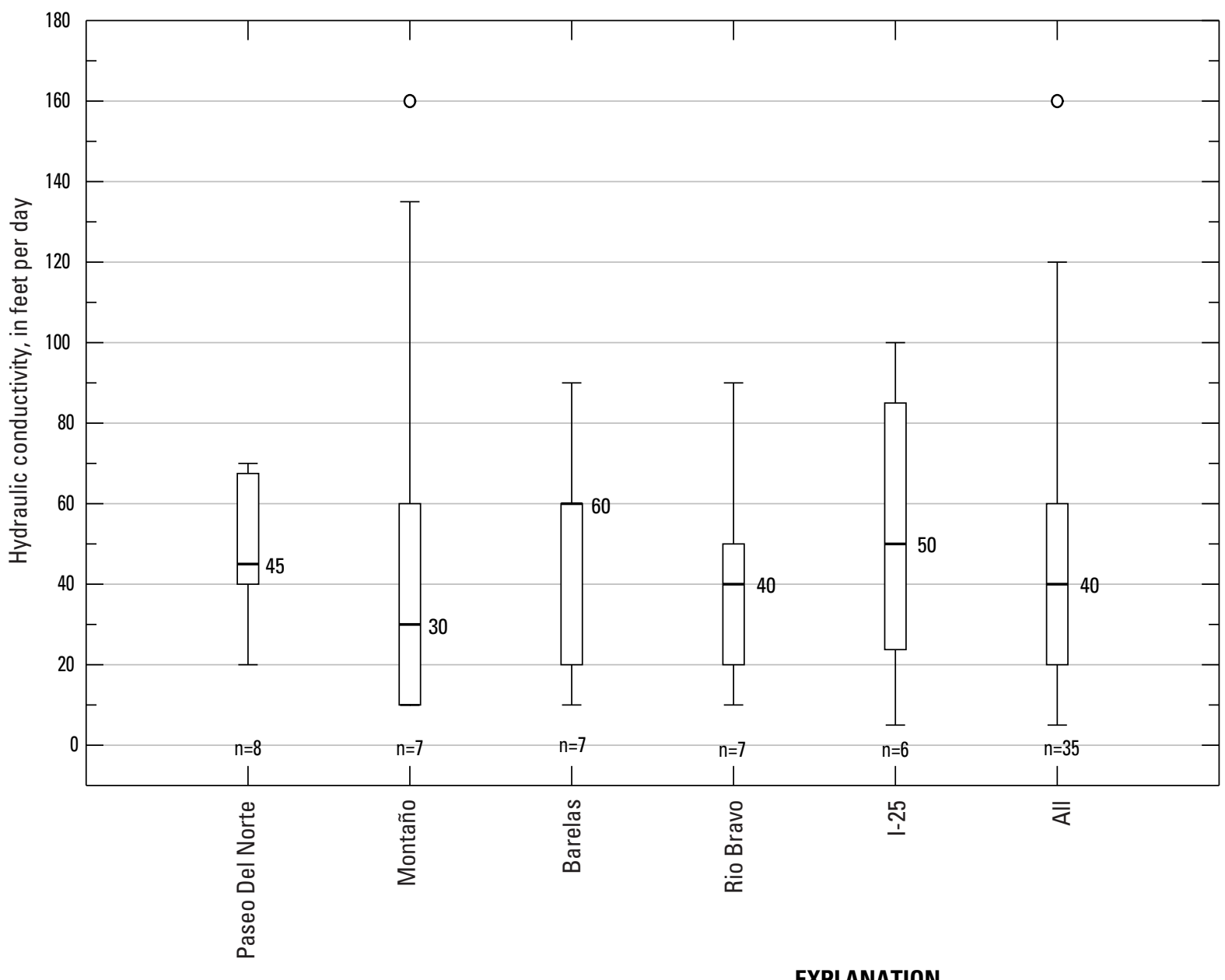

EXPLANATION

outlier (value is between 1.5 and 2 times the interquartile range)

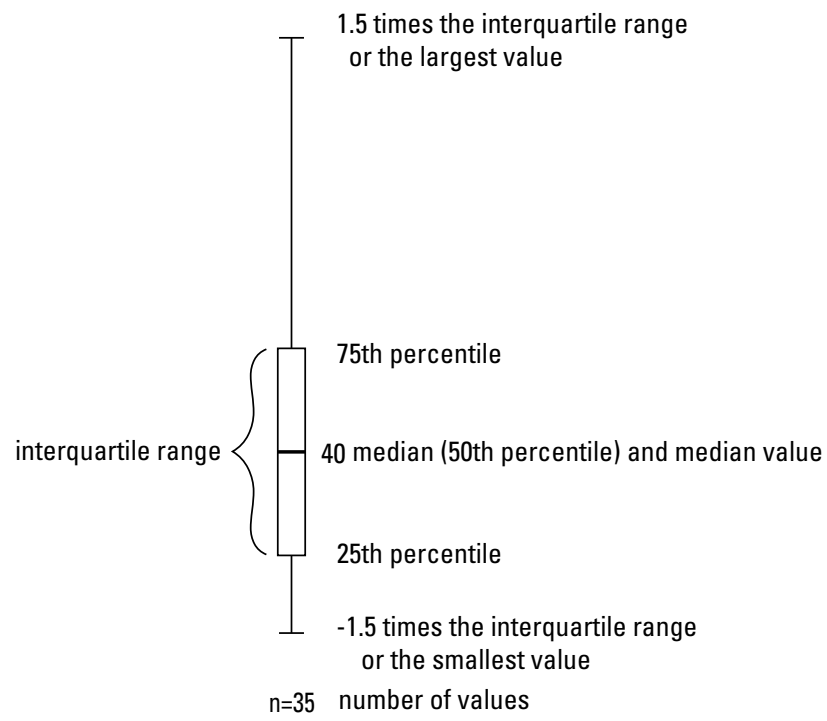

Figure 6. Summary of hydraulic conductivities estimated from slug tests conducted at selected locations in the Rio Grande inner valley alluvial aquifer, Albuquerque, New Mexico. 
Table 2. Summary of Bouwer and Rice (1976) and Butler (1998) slug-test results from piezometers in the Rio Grande inner valley alluvial aquifer, Albuquerque, New Mexico.

[B, Barelas; I, I-25; M, Montaño; P, Paseo del Norte; R, Rio Bravo; a, shallow piezometer; b, mid-depth piezometer; c, deep piezometer; nd, not determined]

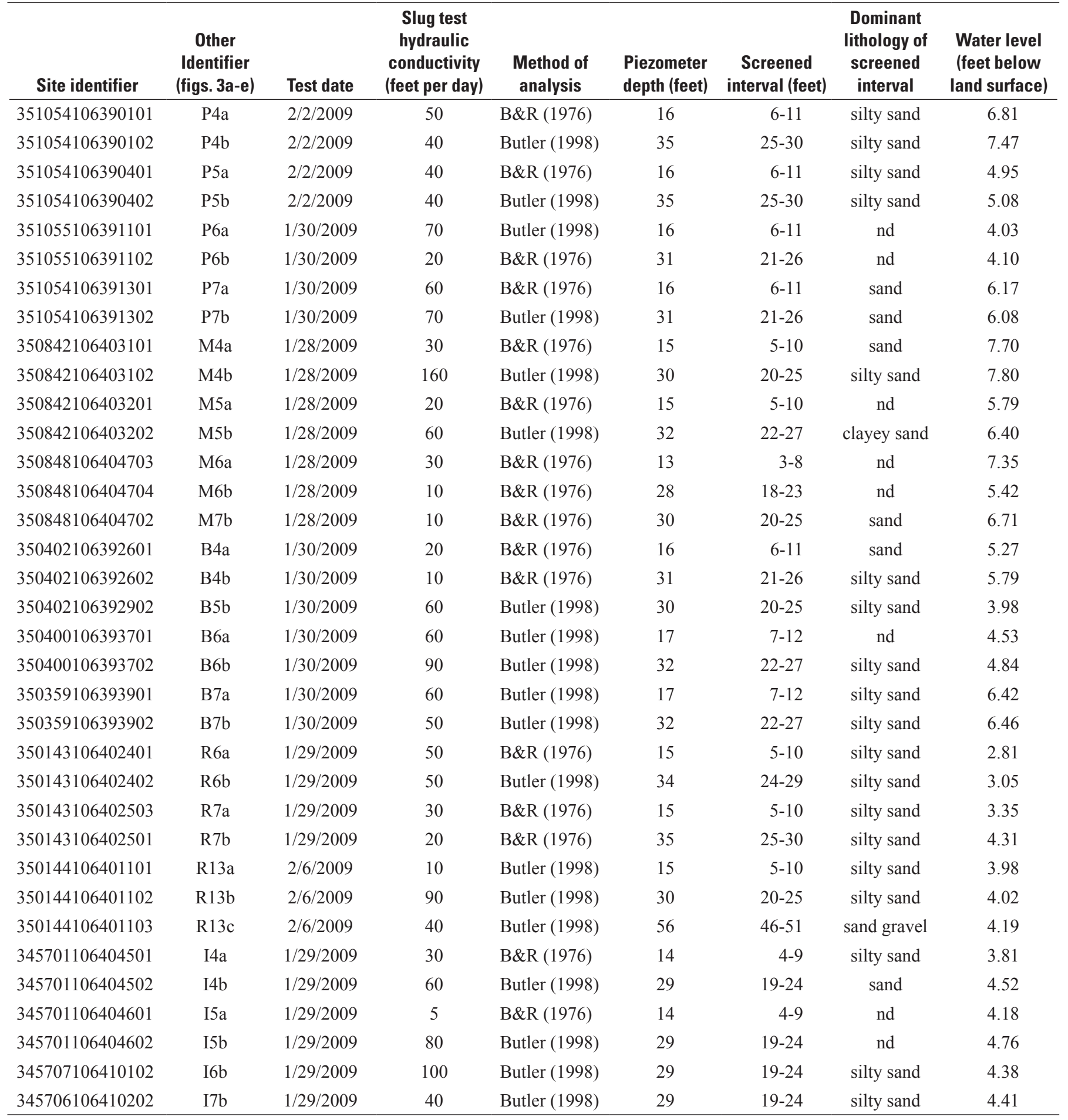



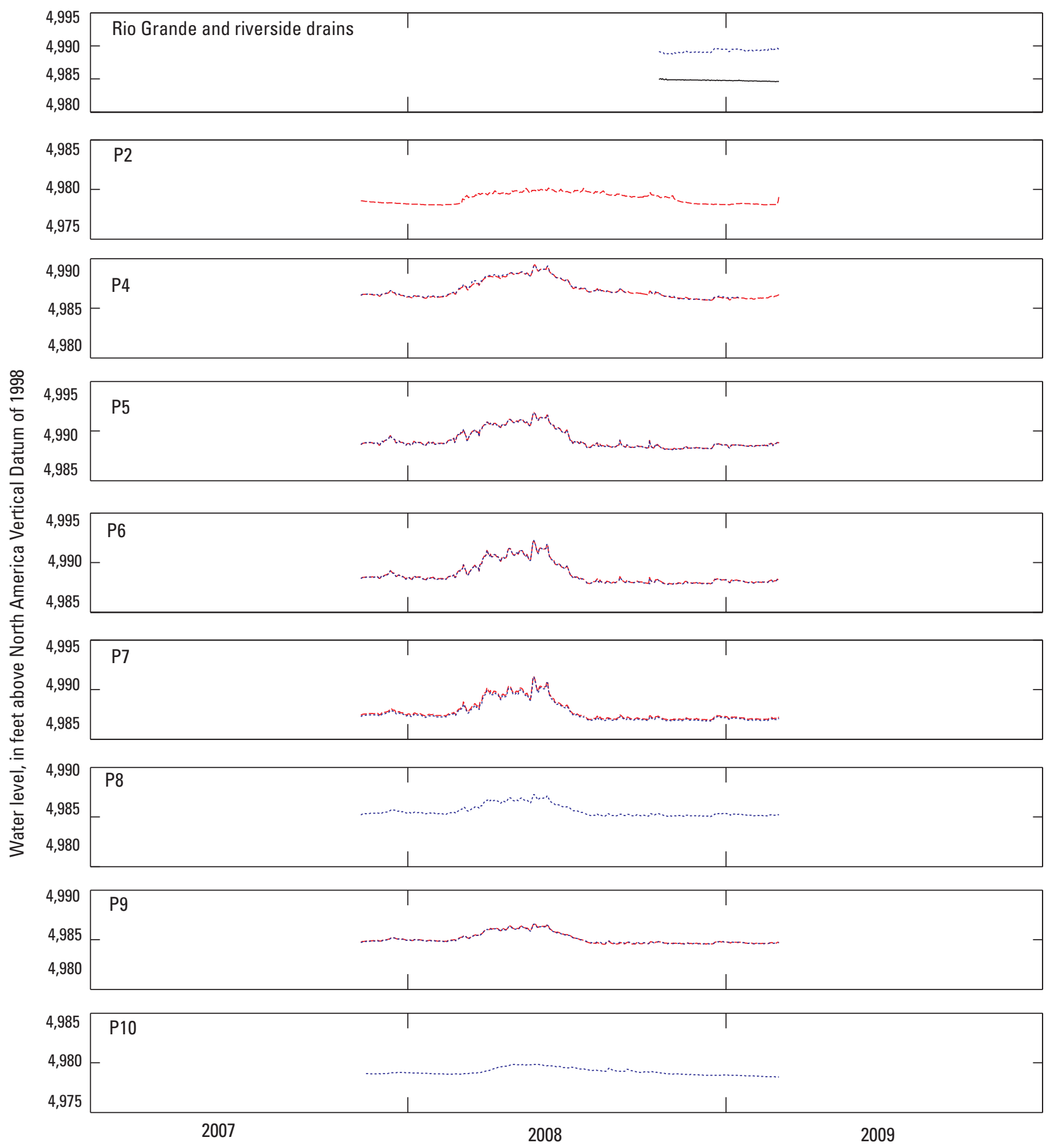

EXPLANATION

Surface water

…-...- Rio Grande

---- East riverside drain

W West riverside drain
Piezometer nests

…-...-. Piezometer a

----- Piezometer b

Piezometer c

Figure 7a. Hydrographs showing daily mean stage of the Rio Grande and riverside drains and daily mean groundwater levels in piezometers at Paseo Del Norte transect 1, November 7, 2007-March 4, 2009. Piezometer locations are shown on figure 3a. 


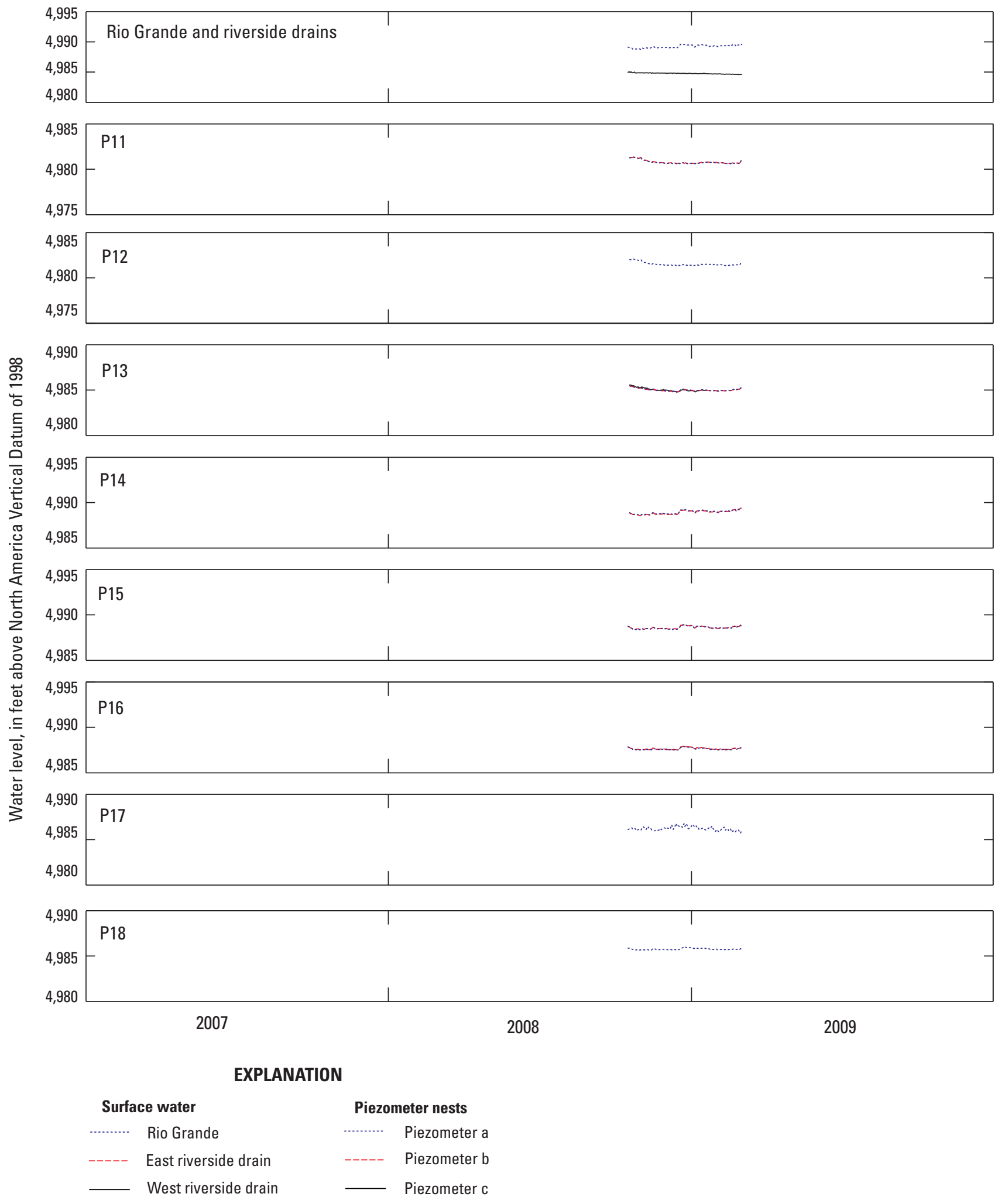

Figure 7b. Hydrographs showing daily mean stage of the Rio Grande and riverside drains and daily mean groundwater levels in piezometers at Paseo del Norte transect 2, October 15, 2008-March 4, 2009. Piezometer locations are shown in figure 3a. 


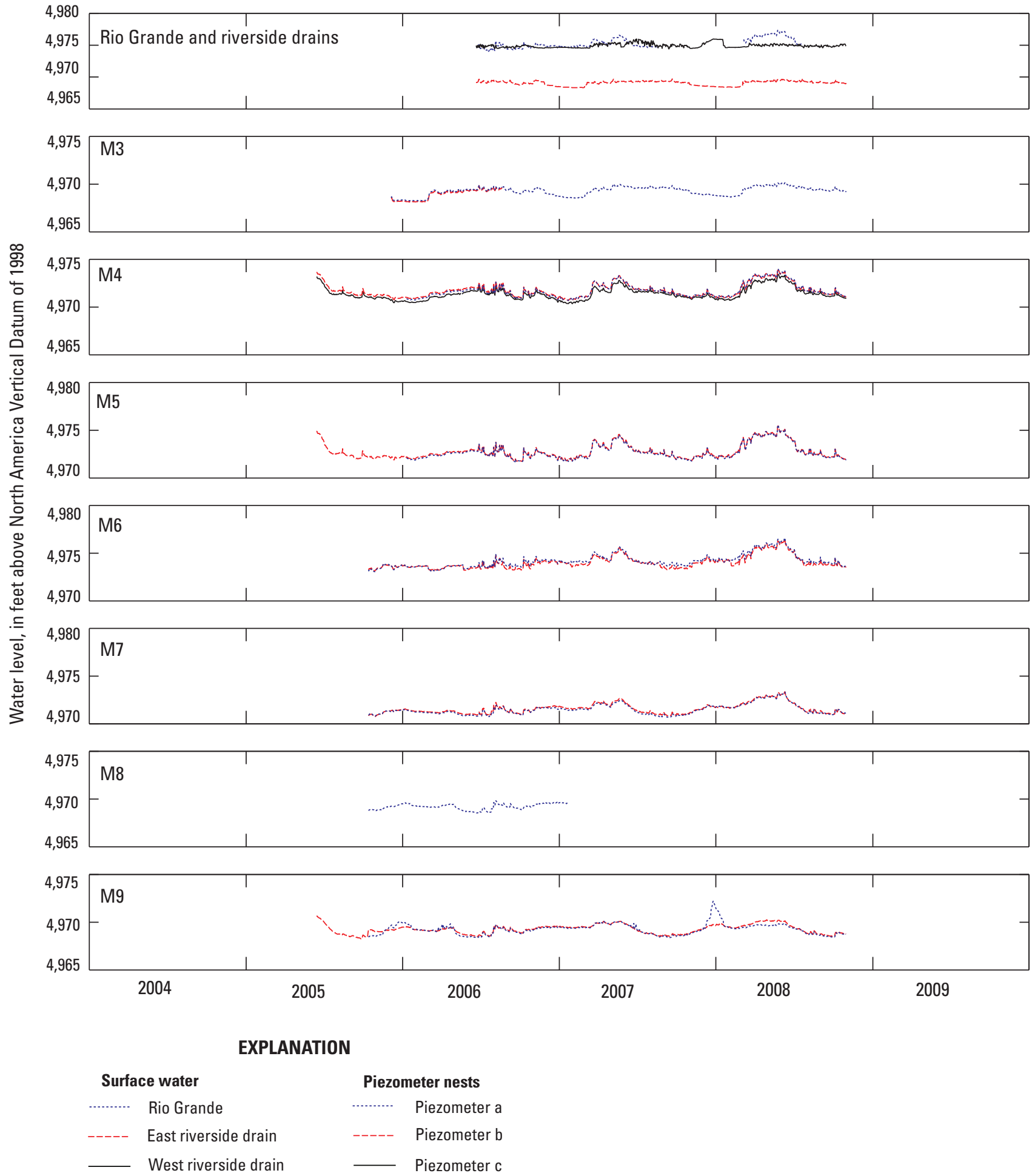

Figure 7c. Hydrographs showing daily mean stage of the Rio Grande and riverside drains and daily mean groundwater levels in piezometers at Montaño transect 1, June 13, 2005-October 31, 2008. Piezometer locations are shown in figure 3b. 


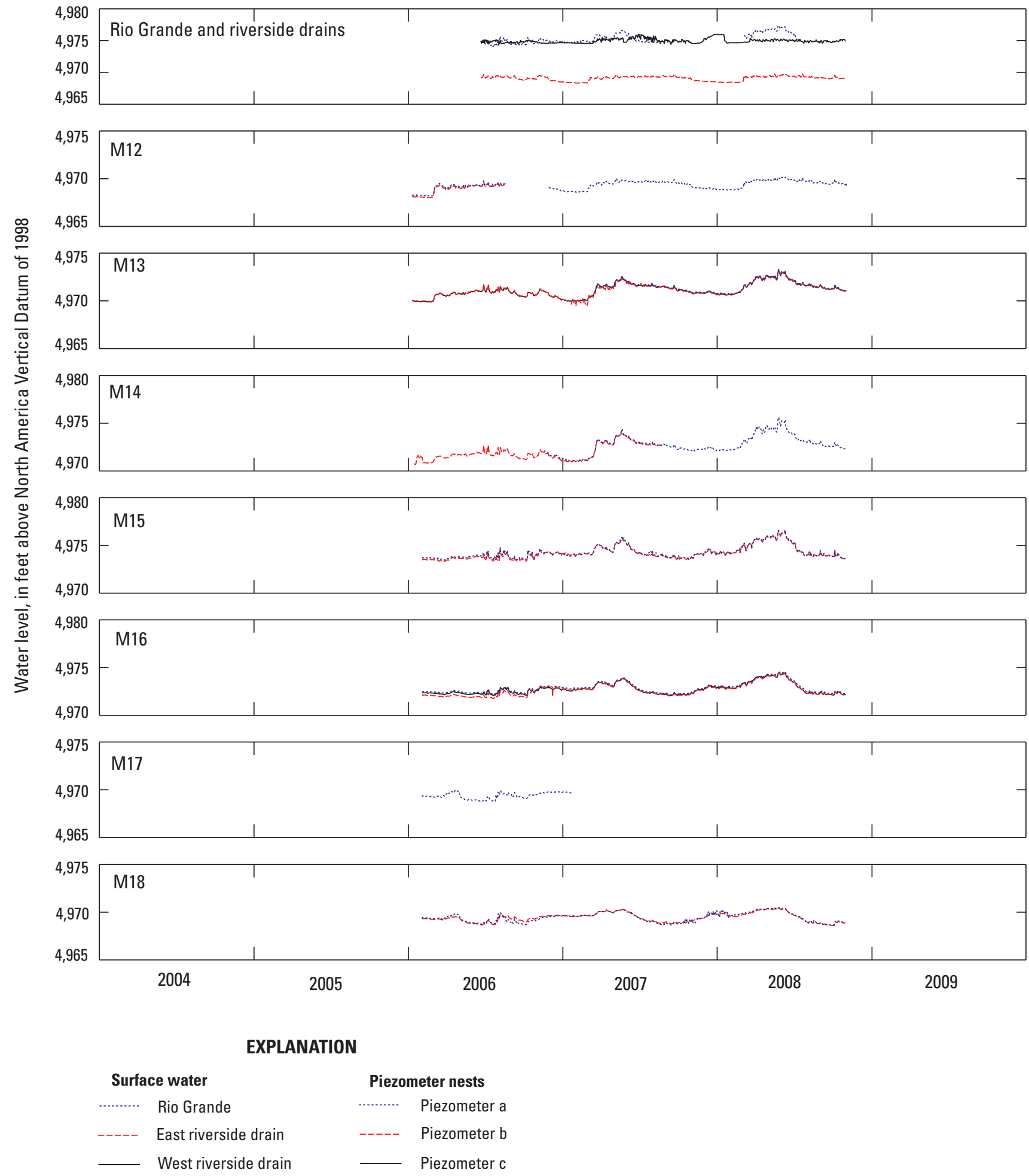

Figure 7d. Hydrographs showing daily mean stage of the Rio Grande and riverside drains and daily mean groundwater levels in piezometers at Montaño transect 2, January 9, 2006-October 31, 2008. Piezometer locations are shown in figure 3b. 


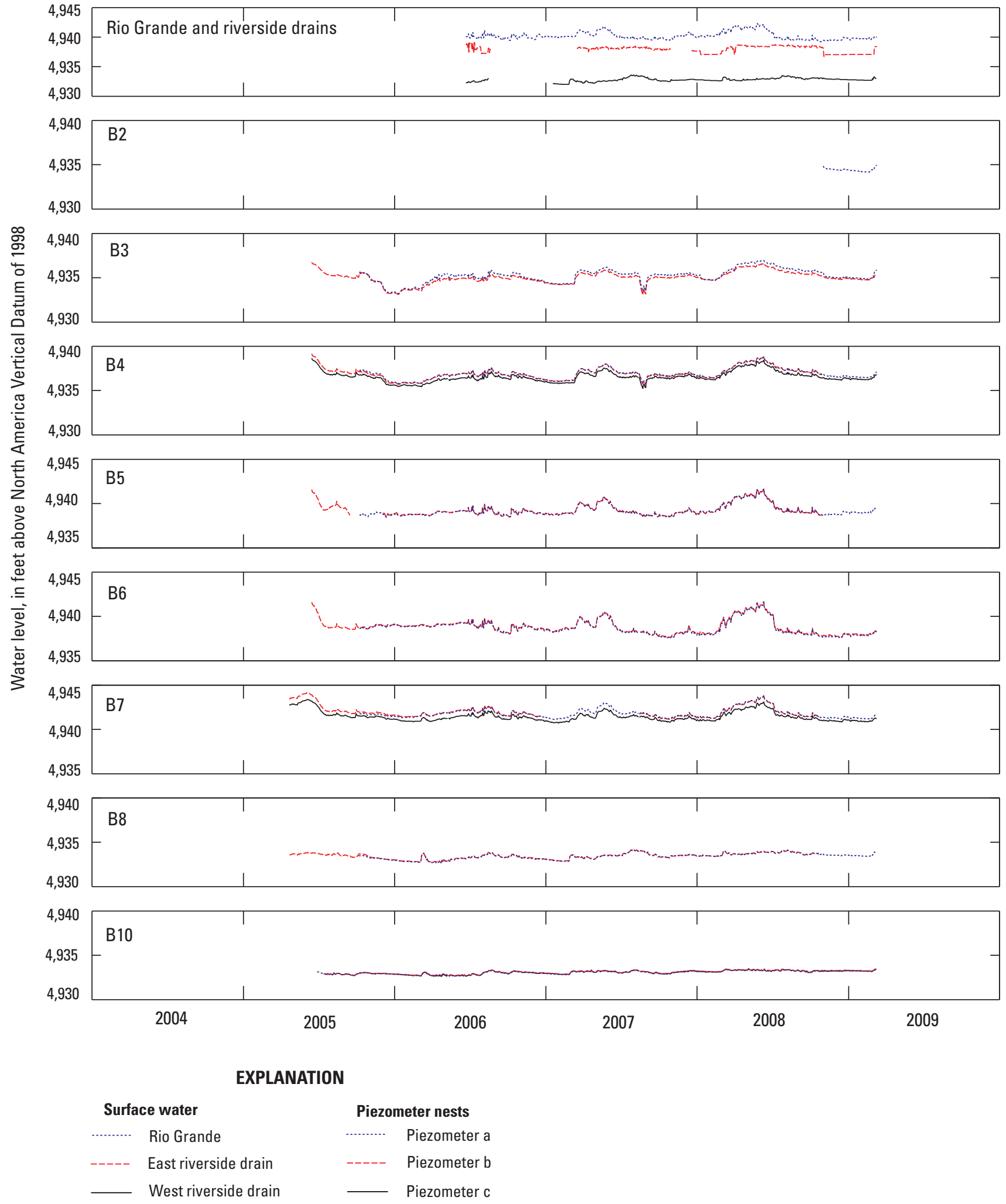

Figure 7e. Hydrographs showing daily mean stage of the Rio Grande and riverside drains and daily mean groundwater levels in piezometers at Barelas transect 1, April 21, 2005-March 10, 2009. Piezometer locations are shown in figure 3c. 


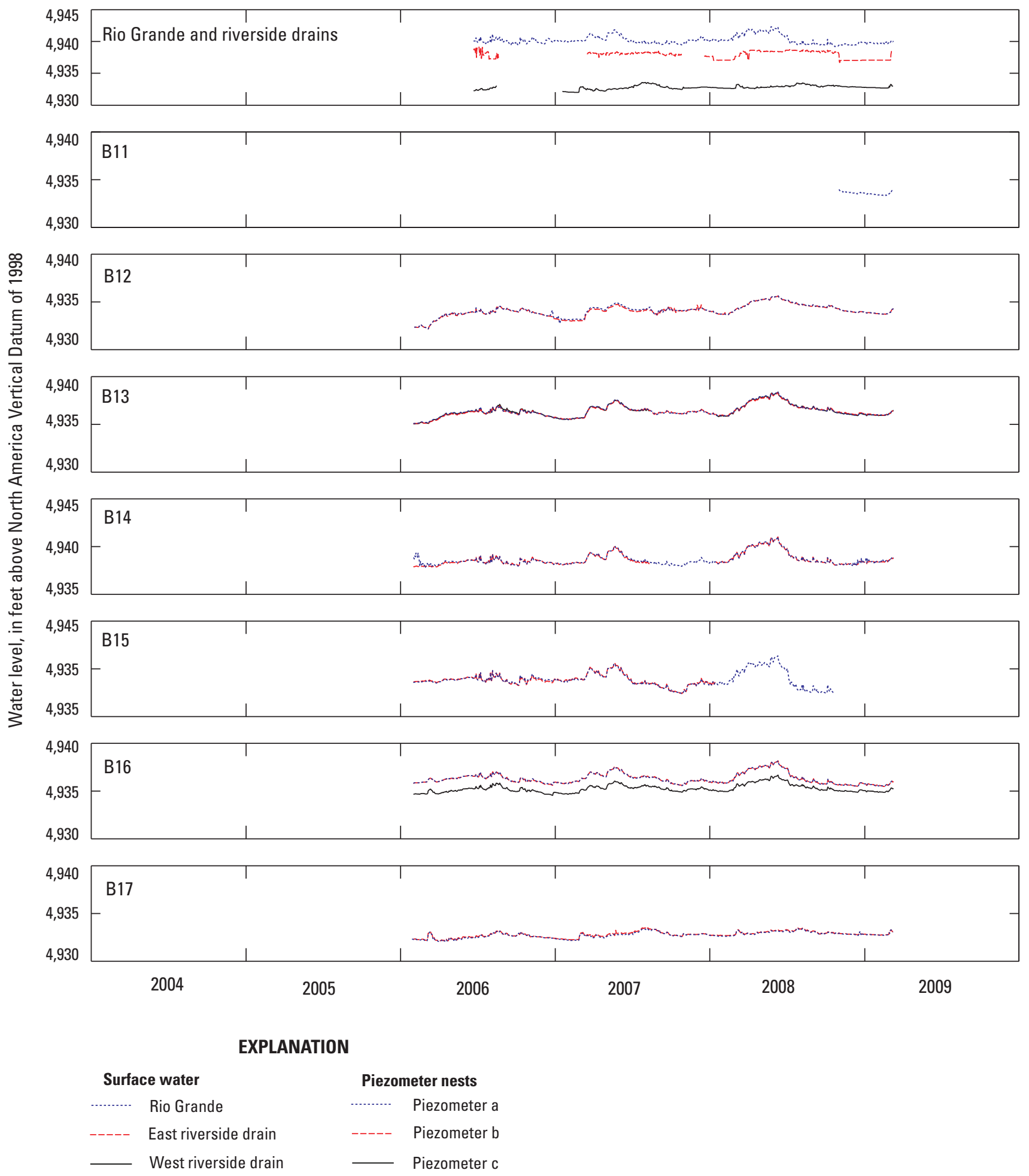

Figure 7f. Hydrographs showing daily mean stage of the Rio Grande and riverside drains and daily mean groundwater levels in piezometers at Barelas transect 2, January 27, 2006-March 10, 2009. Piezometer locations are shown in figure 3c. 


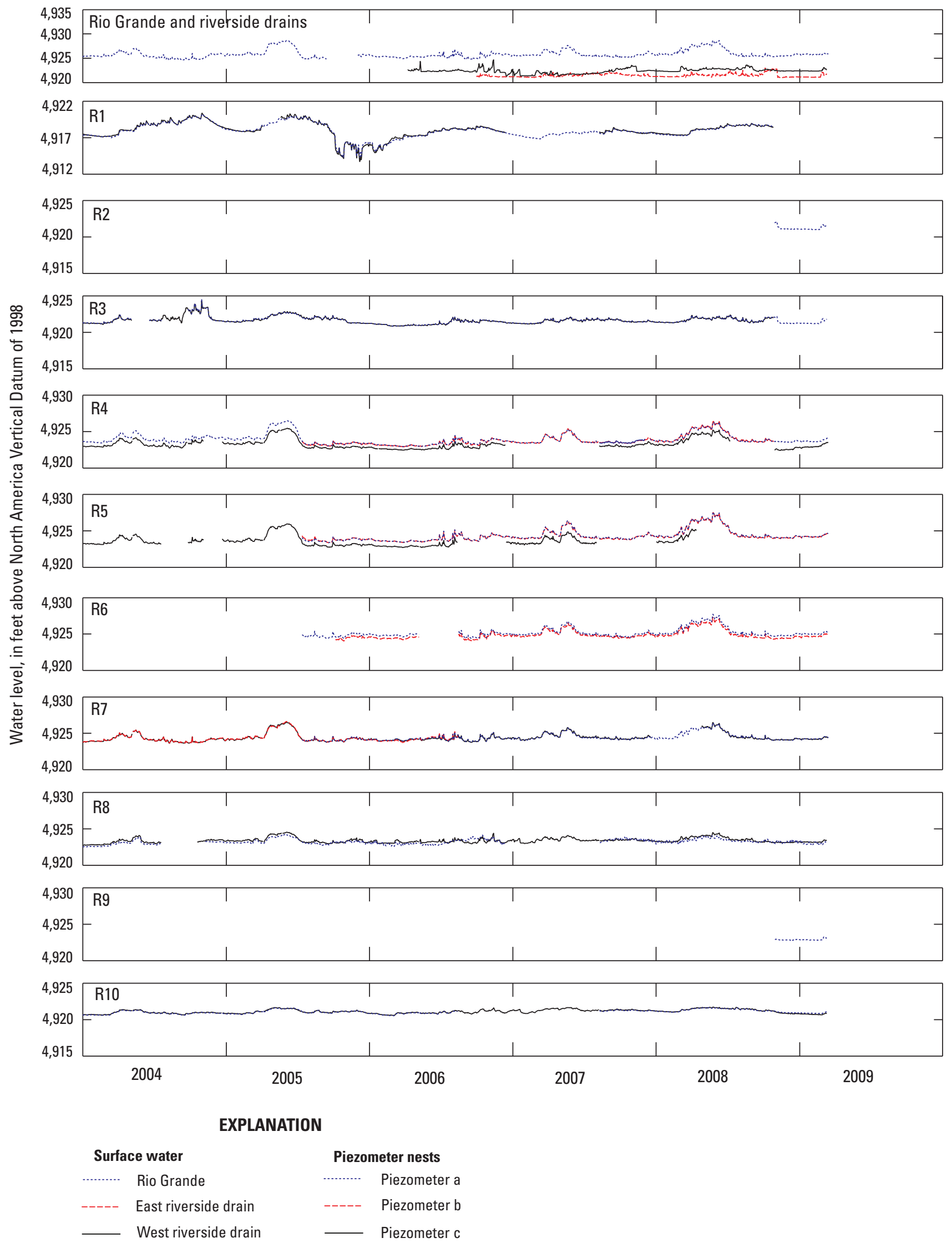

Figure 7g. Hydrographs showing daily mean stage of the Rio Grande and riverside drains and daily mean groundwater levels in piezometers at Rio Bravo transect 1, January 1, 2004-March 15, 2009. Piezometer locations are shown in figure 3d. 


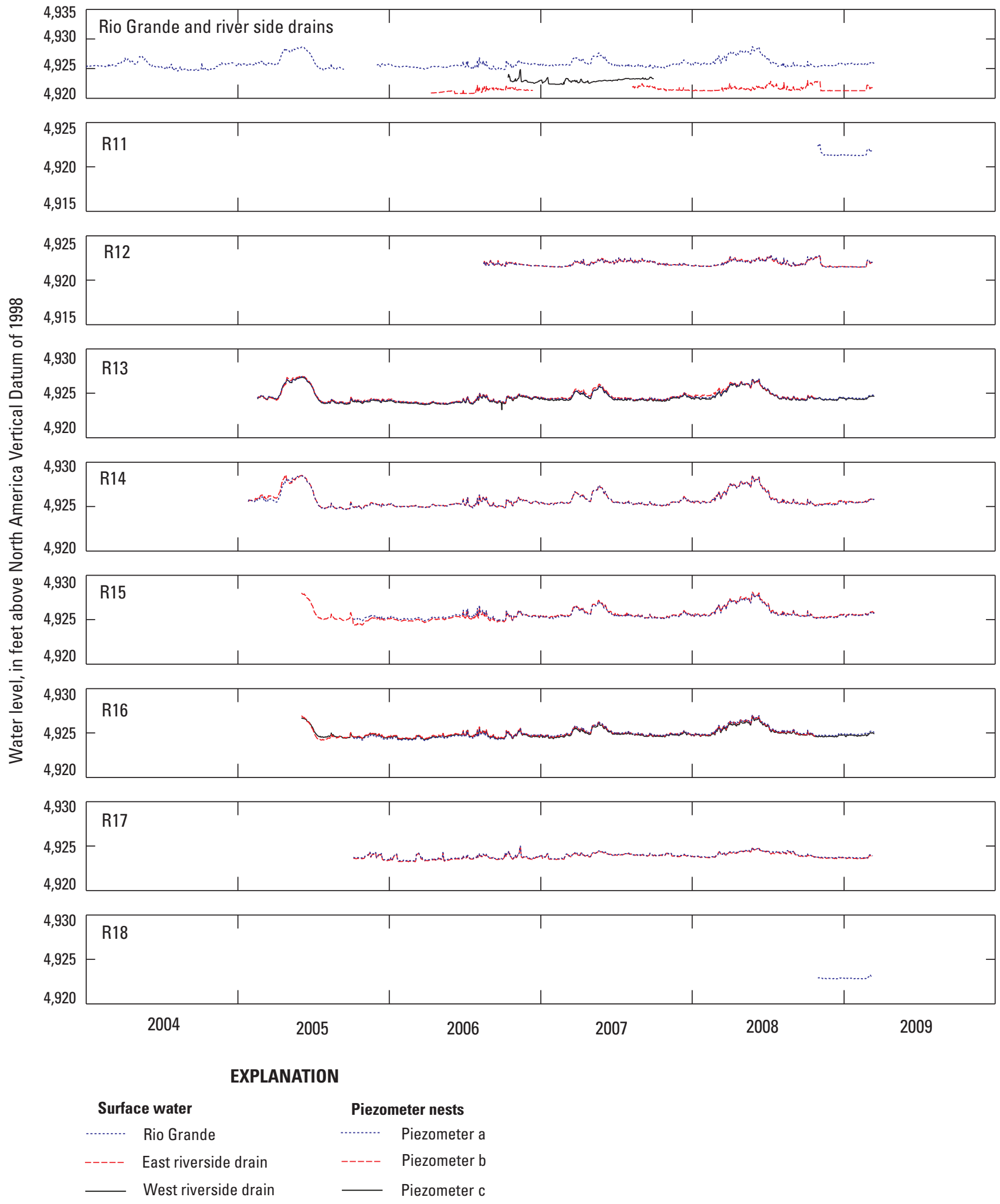

Figure 7h. Hydrographs showing daily mean stage of the Rio Grande and riverside drains and daily mean groundwater levels in piezometers at Rio Bravo transect 2, January 1, 2004-March 15, 2009. Piezometer locations are shown in figure 3d. 


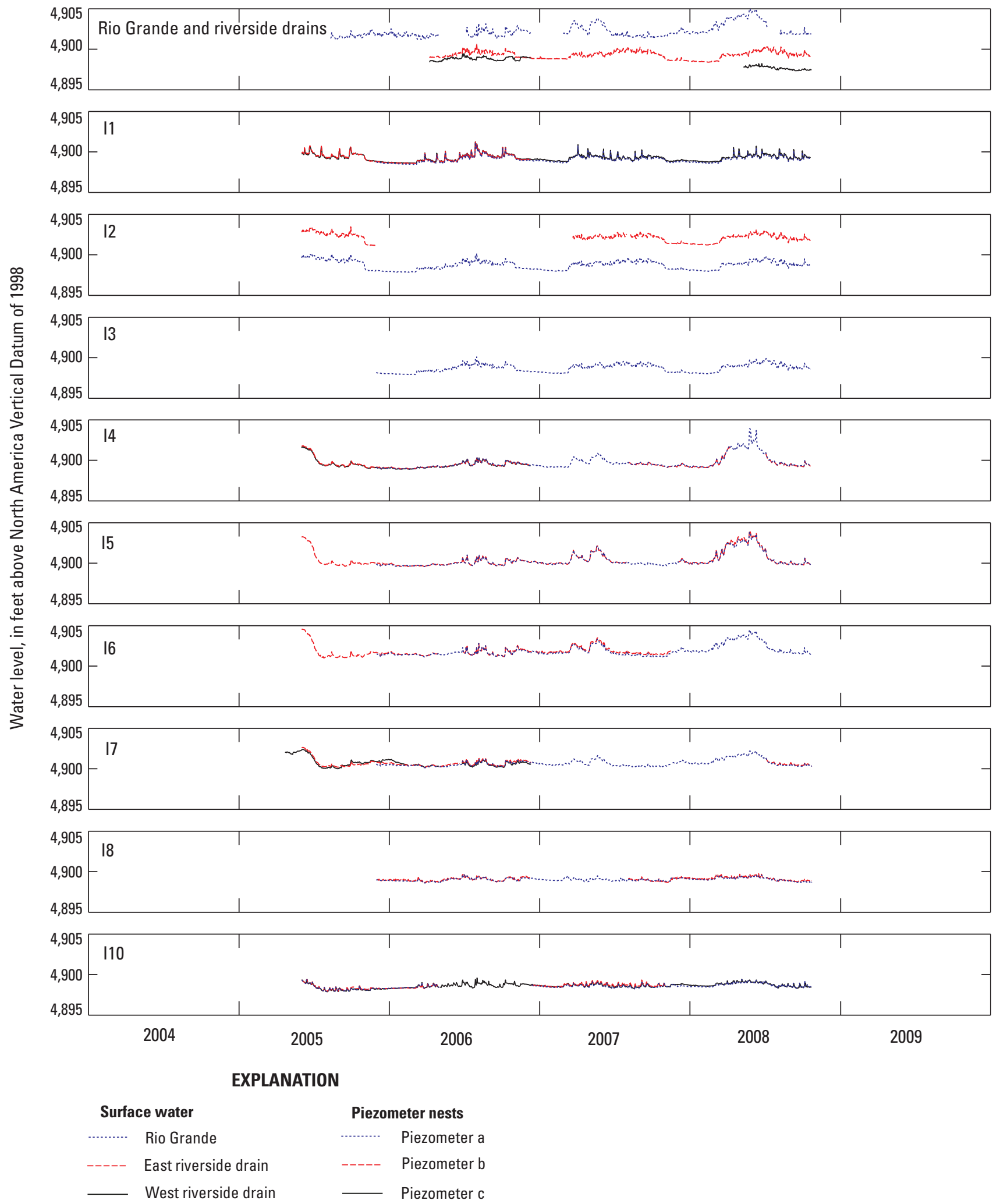

Figure 7i. Hydrographs showing daily mean stage of the Rio Grande and riverside drains and daily mean groundwater levels in piezometers at I-25 transect 1, April 22, 2005-October 24, 2008. Piezometer locations are shown in figure 3e. 


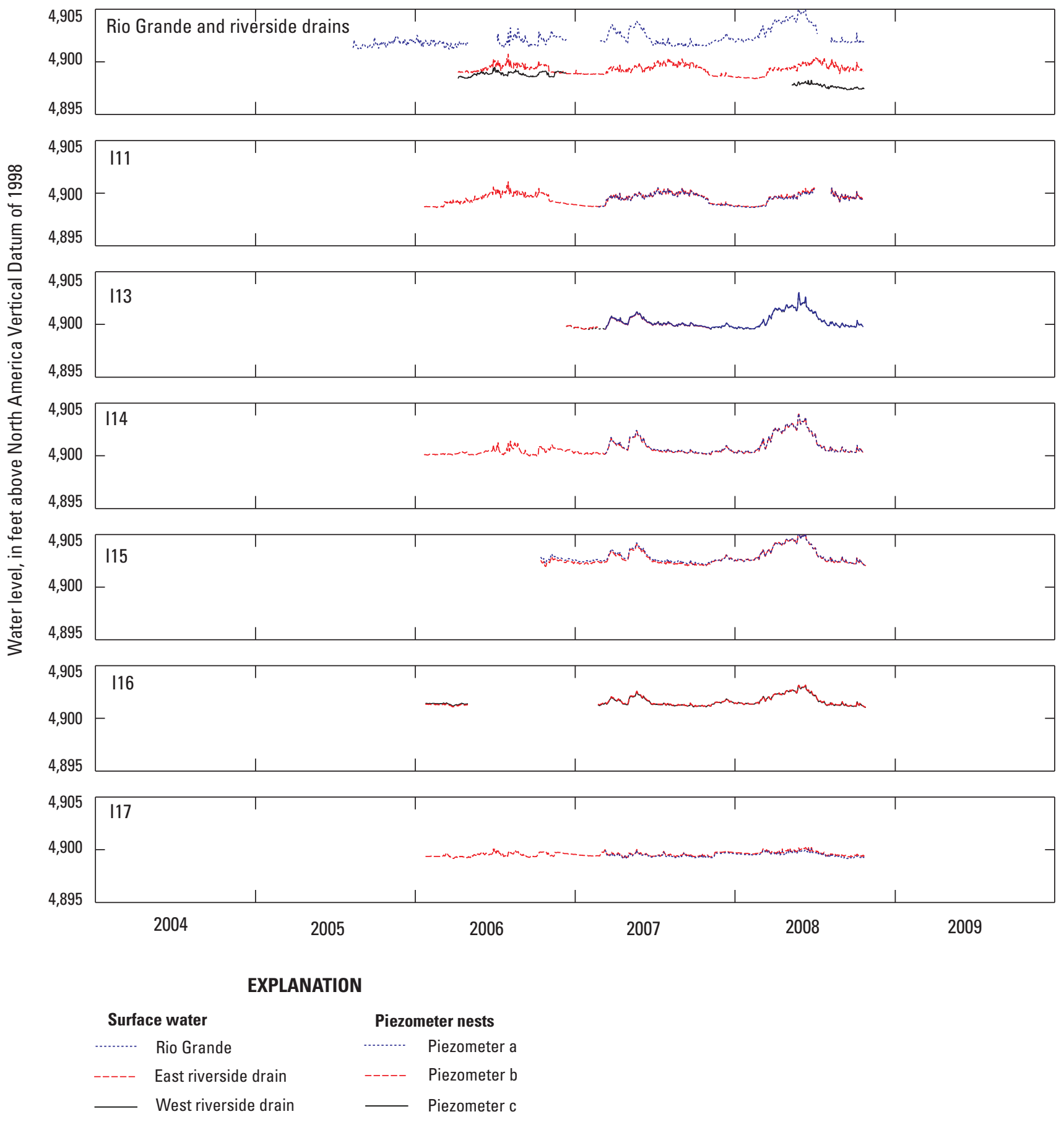

Figure 7j. Hydrographs showing daily mean stage of the Rio Grande and riverside drains and daily mean groundwater levels in piezometers at I-25 transect 2, August 10, 2005-October 27, 2008. Piezometer locations are shown in figure 3e. 
Table 3. Median annual magnitude of groundwater horizontal hydraulic gradient and direction of groundwater flow at piezometer transects, Rio Grande inner valley alluvial aquifer, Albuquerque, New Mexico, 2006-9.

[Site locations shown in figs. 3a-e; CW, clockwise from downstream direction of channel; $\mathrm{CCW}$, counter clockwise from downstream direction of channel. Groundwater flow direction is relative to the downstream direction of the Rio Grande channel at each transect.]

\begin{tabular}{lcc}
\hline $\begin{array}{c}\text { Transect } \\
\text { location }\end{array}$ & $\begin{array}{c}\text { Median annual } \\
\text { horizontal hydraulic } \\
\text { gradient (feet per foot) }\end{array}$ & $\begin{array}{c}\text { Direction of } \\
\text { groundwater flow } \\
\text { (degrees) }\end{array}$ \\
\hline East & Paseo Del Norte & \\
West & 0.010 & $89 \mathrm{CCW}$ \\
& .005 & $78 \mathrm{CW}$ \\
\hline East & Montaño & \\
West & .010 & $89 \mathrm{CCW}$ \\
\hline East & .003 & $77 \mathrm{CW}$ \\
West & Barelas & $87 \mathrm{CCW}$ \\
\hline East & .009 & $77 \mathrm{CW}$ \\
West & .011 & $73 \mathrm{CCW}$ \\
\hline East & Rio Bravo & $76 \mathrm{CW}$ \\
\hline West & .005 & $71 \mathrm{CCW}$ \\
\hline
\end{tabular}

The effect of this loss of water from the drain was not evident in the February 7, 2008 groundwater-level data and so is not reflected in the water-level contours (fig. 3b). At the Barelas transects, the water level at BS1 in the Albuquerque Riverside Drain was more than $2 \mathrm{ft}$ higher than the water levels in piezometers B3a and B12a on February 7, 2008 (fig. 3c). These water-level differences are attributable to a point of diversion structure in the drain about $900 \mathrm{ft}$ downstream from B12a that obstructs flow and elevates the water level in the drain so that the water level in the drain upstream from the diversion was substantially higher than downstream from the structure.

Hourly groundwater temperatures were recorded at depths of 10 and $20 \mathrm{ft}$ in selected piezometer nests. Daily mean water temperature for all measurement points are shown in figures $8 \mathrm{a}-8 \mathrm{j}$. Large ranges in surface-water temperature are apparent: surface-water temperatures ranged from $37^{\circ} \mathrm{F}$ in the winter to $79^{\circ} \mathrm{F}$ in the summer. Surface-water temperatures in the drains typically were similar to temperatures measured in the Rio Grande, but the magnitude of fluctuations in the river were somewhat larger. Maximum and minimum water temperatures in piezometers generally indicate a decrease in amplitude and an increase in time lag of the temperature signal with increasing depth and distance from the river. High frequency temperature fluctuations of a few degrees in shallow piezometers installed adjacent to the river rapidly dissipated with depth or distance from the river. For example, the high frequency temperature fluctuations that are evident in piezometer P5a, next to the river, are not evident in piezometers $\mathrm{P} 4 \mathrm{a}$ and $\mathrm{P} 3 \mathrm{a}$ (fig. 8a), which are farther away from the river (fig. 3a). The high-frequency fluctuations were not recorded in mid-depth piezometers or shallow piezometers located greater than a few hundred feet from the river. For example, short-term temperature variations recorded in M15a, a shallow piezometer adjacent to the river, are substantially attenuated in M15b, an intermediate-depth piezometer a few feet from M15a, and are not evident in M16b, 400 to $500 \mathrm{ft}$ to the west of M15a (fig. 8d).

Vertical temperature profiles were collected during the months of October 2008 and January and February 2009 in the deepest piezometer at selected piezometer nests during the nonirrigation season (fig. 9). Temperature-profile data were collected to evaluate the depth of the alluvial aquifer that is influenced by leakage from the river. Temperatures were recorded at 5 -ft intervals from about $0.5 \mathrm{ft}$ below the water surface to the bottom of each piezometer. Although piezometers were constructed with a screened interval of $5 \mathrm{ft}$ near the bottom of the piezometer, the temperature of water in blank (nonscreened) casing was assumed to be the same as the temperature of water outside the casing. Groundwatertemperature profiles in figure 9 form envelopes that generally are bracketed by warmest temperatures in October and coolest temperatures in February. Groundwater temperatures recorded throughout the nonirrigation season were most variable at depths less than $30 \mathrm{ft}$ and generally ranged from 40 to $70^{\circ} \mathrm{F}$.

Temperature envelopes in figure 9 generally can be classified as fan- or tulip-shaped depending on the direction and velocity of groundwater flow. At M7, M9, R3, R8, I3, I4, and I5 the fan-shape of the temperature envelope shows the seasonal temperature extinction depth (the depth at which seasonal temperature variations are not observed) during the nonirrigation season ranges from 20 to $30 \mathrm{ft}$. The temperature ranges near the water table in these fanshaped envelopes generally were $20^{\circ} \mathrm{F}$ or less for the period between October 2008 and February 2009. The compressed nature of fan-shaped temperature envelopes is indicative of areas where (1) vertical groundwater flow is limited, and (2) horizontal advective transport of heat is relatively uniform. At the remaining piezometer nests, temperature ranges near the water table generally were greater than $20^{\circ} \mathrm{F}$ for the period between October 2008 and February 2009. These temperature envelopes generally are more tulip-like in shape with seasonal temperature extinction depths below the depths of observation and broad changes in temperature throughout the depths of observation. Temperature inflections with depth in tulip-shaped temperature envelopes show sharp changes in heat fluxes associated with heterogeneous groundwater-flow patterns (Constantz and others, 2003). 


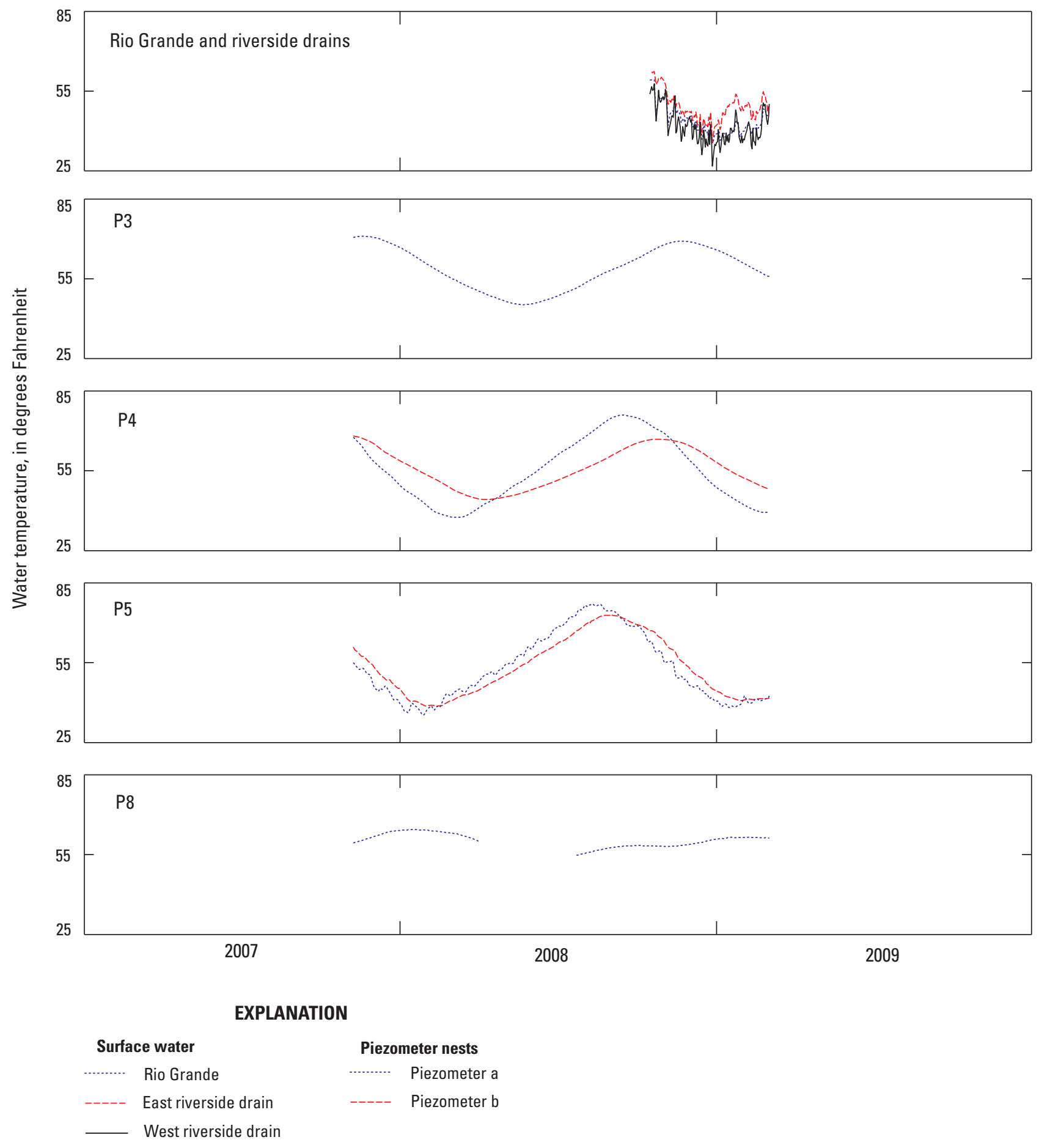

Figure 8a. Daily mean water temperature in the Rio Grande and riverside drains and daily mean groundwater temperature in piezometers at Paseo del Norte transect 1, November 7, 2007-March 4, 2009. Piezometer locations are shown on figure 3a. 


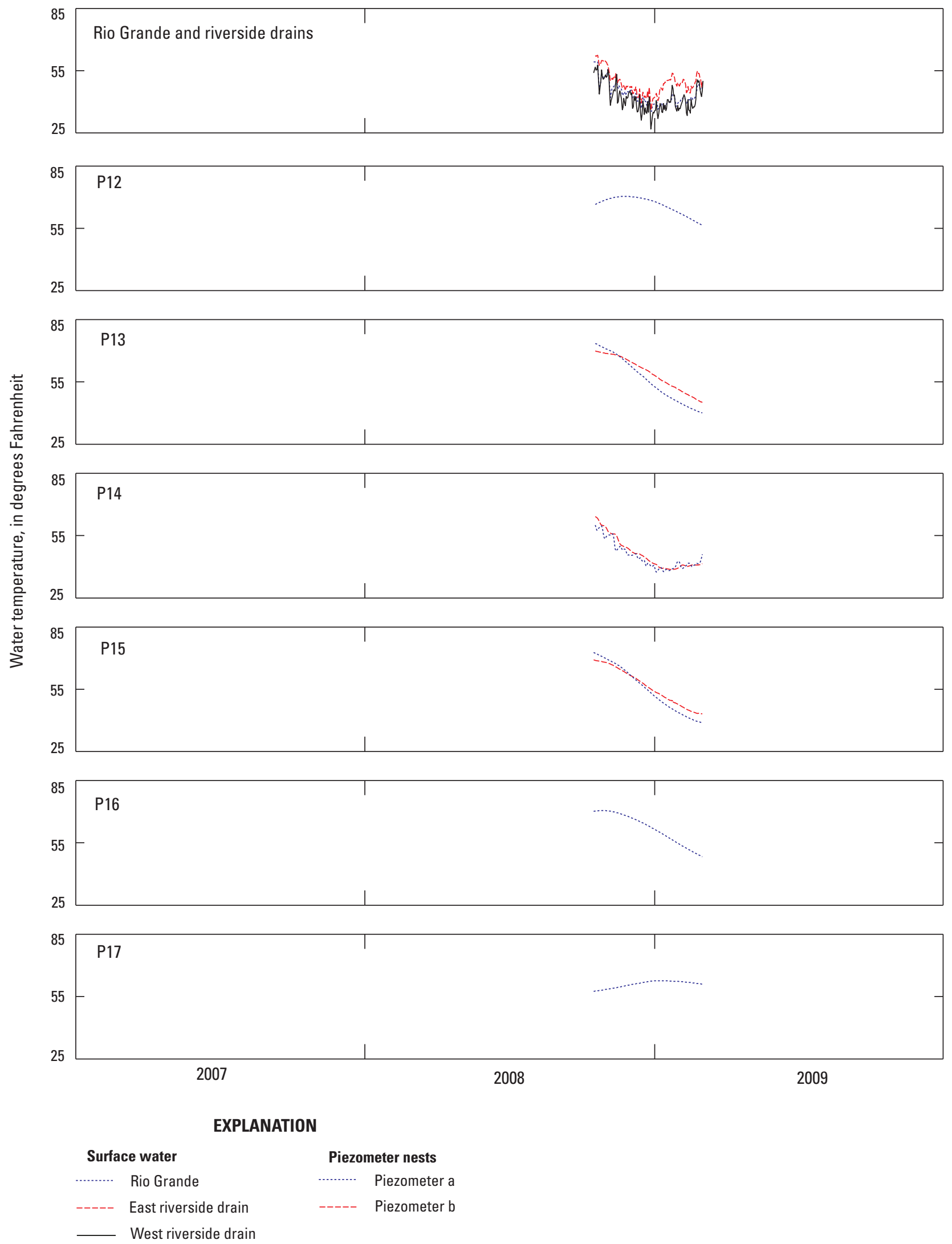

Figure 8b. Daily mean water temperature in the Rio Grande and riverside drains and daily mean groundwater temperature in piezometers at Paseo Del Norte transect 2, October 15, 2007-March 4, 2009. Piezometer locations are shown on figure 3 a. 

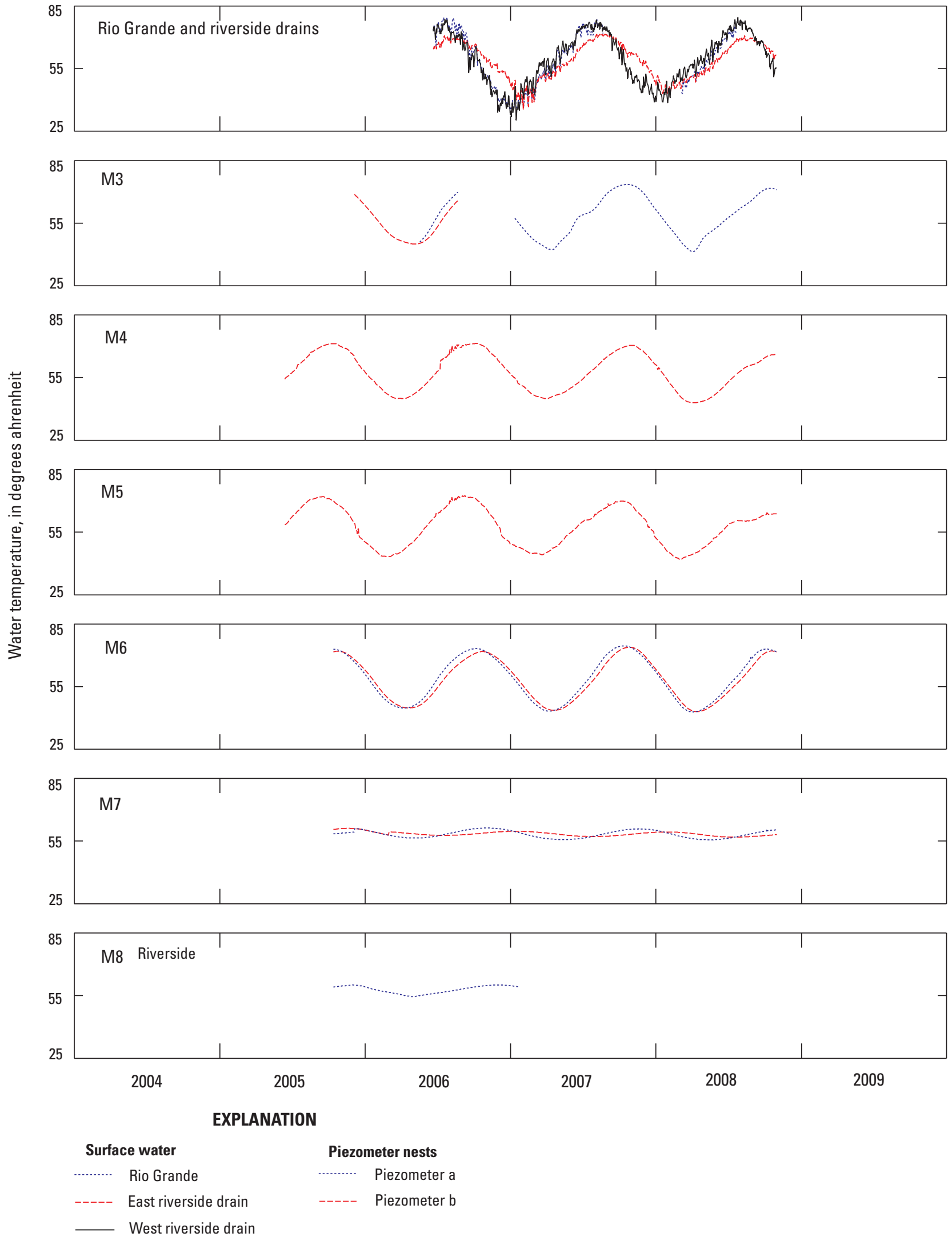

Figure 8c. Daily mean water temperature in the Rio Grande and riverside drains and daily mean groundwater temperature in piezometers at Montaño transect 1, June 13, 2005-0ctober 31, 2008. Piezometer locations are shown on figure 3b. 


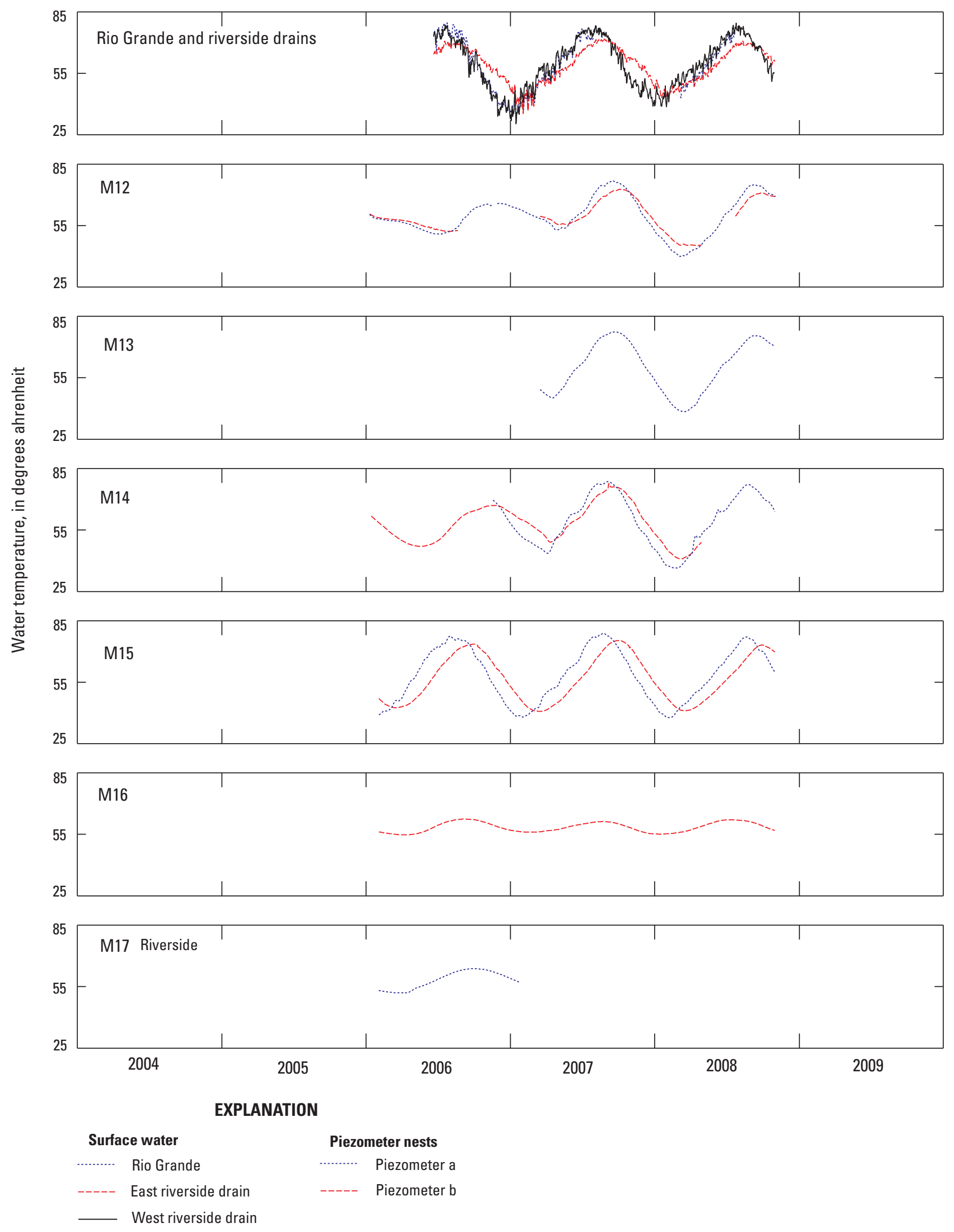

Figure 8d. Daily mean water temperature in the Rio Grande and riverside drains and daily mean groundwater temperature in piezometers at Montaño transect 2, January 9, 2006-October 31, 2008. Piezometer locations are shown on figure 3b. 


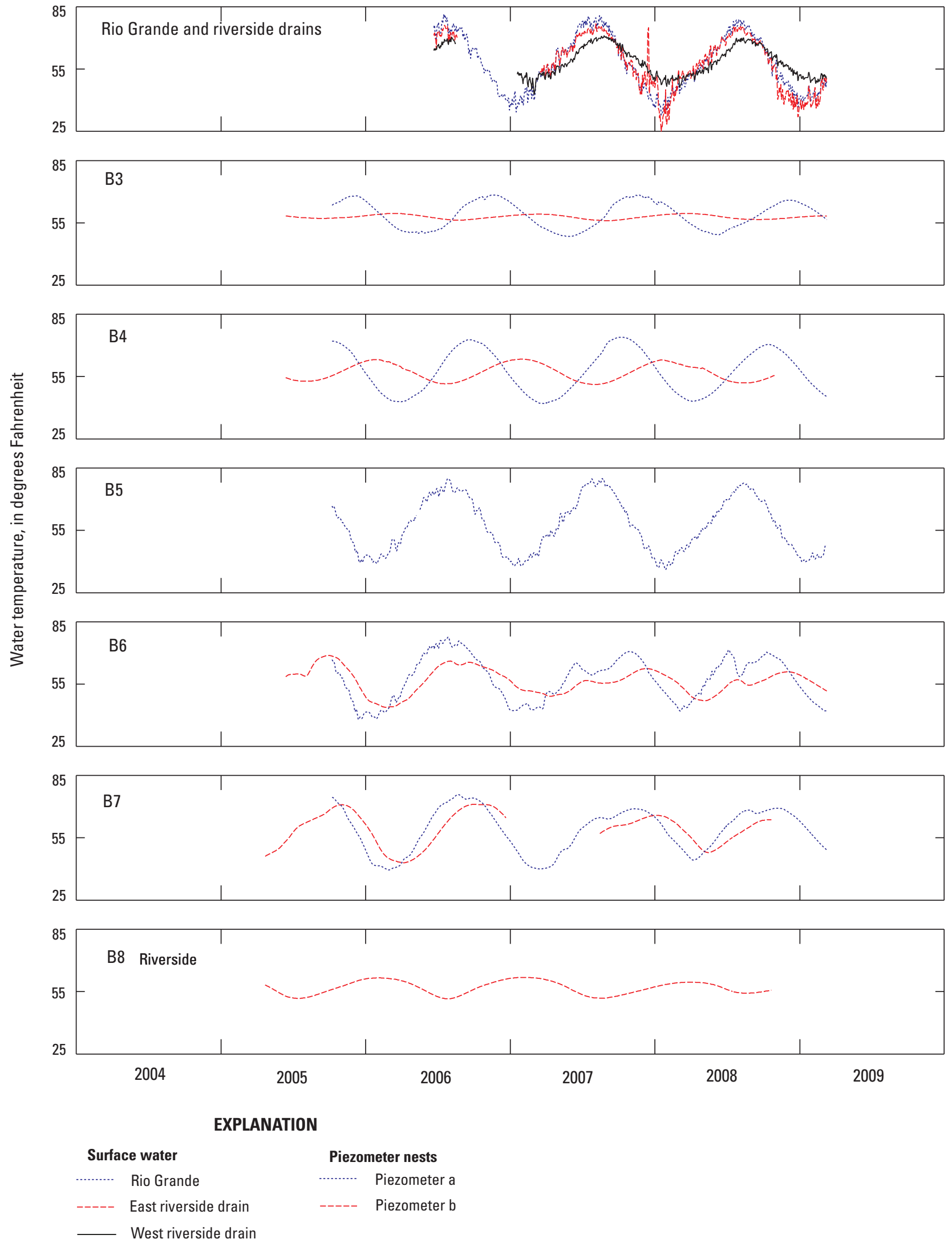

Figure 8e. Daily mean water temperature in the Rio Grande and riverside drains and daily mean groundwater temperature in piezometers at Barelas transect 1, April 21, 2005-March 10, 2009. Piezometer locations are shown on figure 3c. 


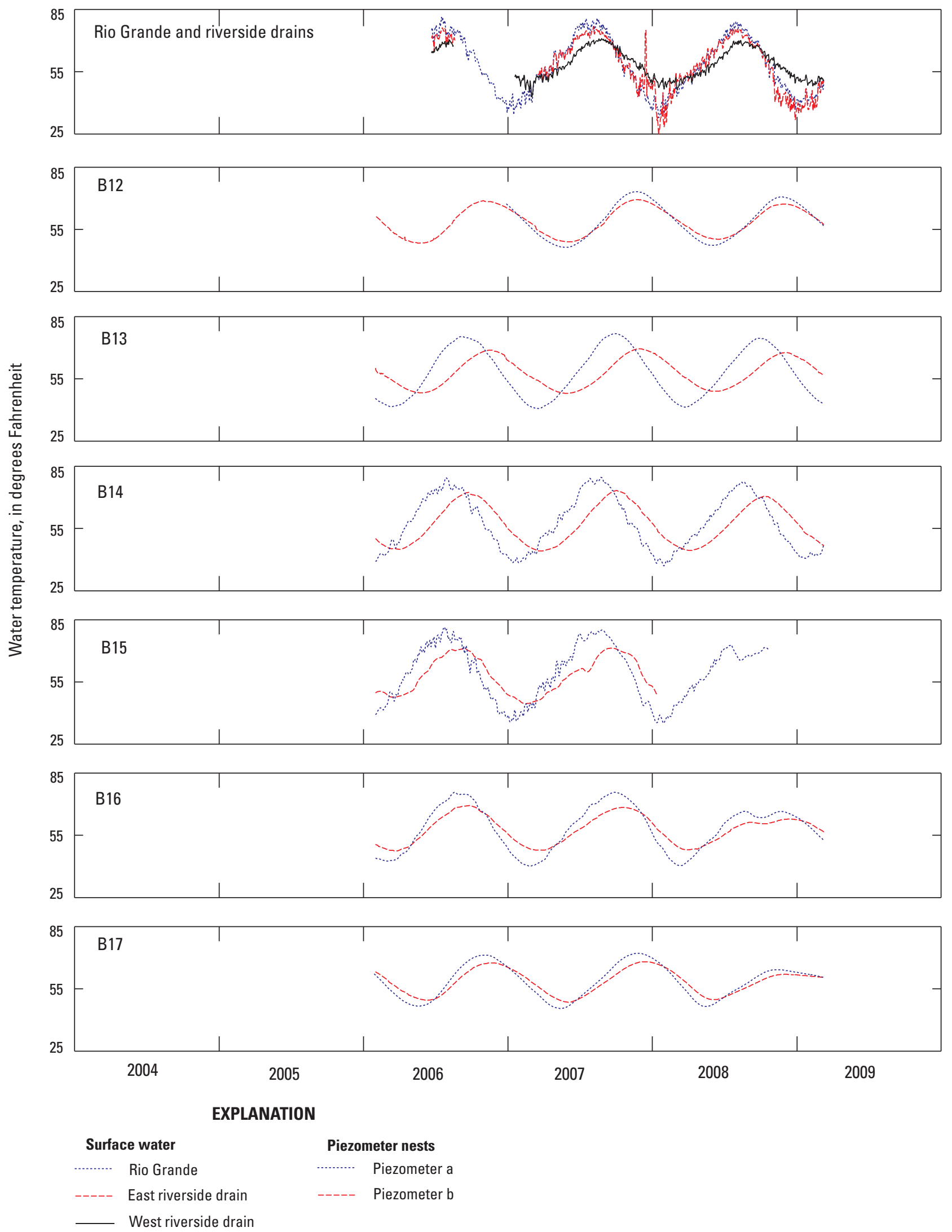

Figure 8f. Daily mean water temperature in the Rio Grande and riverside drains and daily mean groundwater temperature in piezometers at Barelas transect 2, January 27, 2006-March 10, 2009. Piezometer locations are shown on figure 3c. 

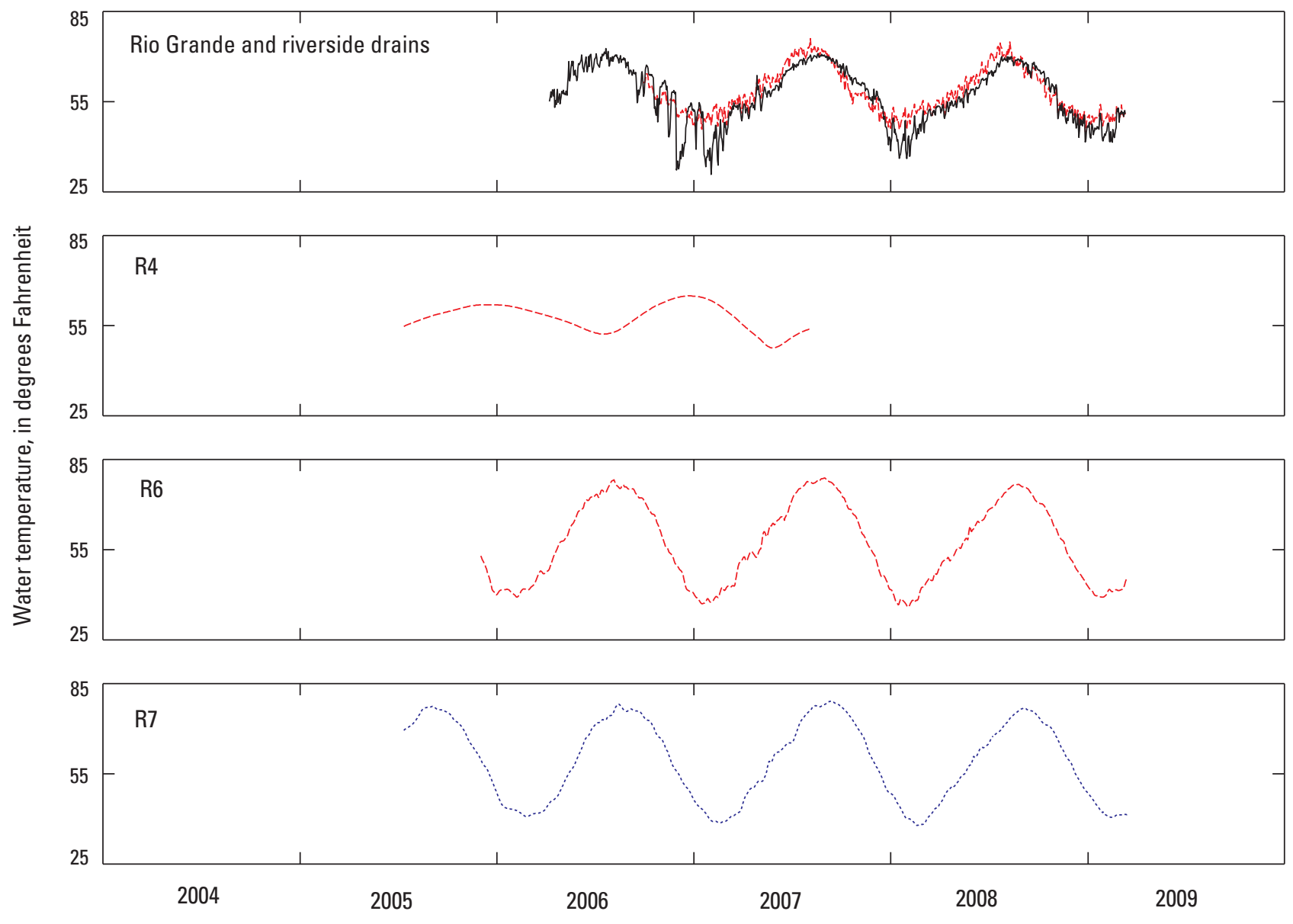

EXPLANATION

Surface water

...-..... Rio Grande

East riverside drain

West riverside drain
Piezometer nests

Piezometer a

Piezometer b

Figure 8g. Daily mean water temperature in the Rio Grande and riverside drains and daily mean groundwater temperature in piezometers at Rio Bravo transect 1, June 3, 2005-March 15, 2009. Piezometer locations are shown on figure 3d. 
85
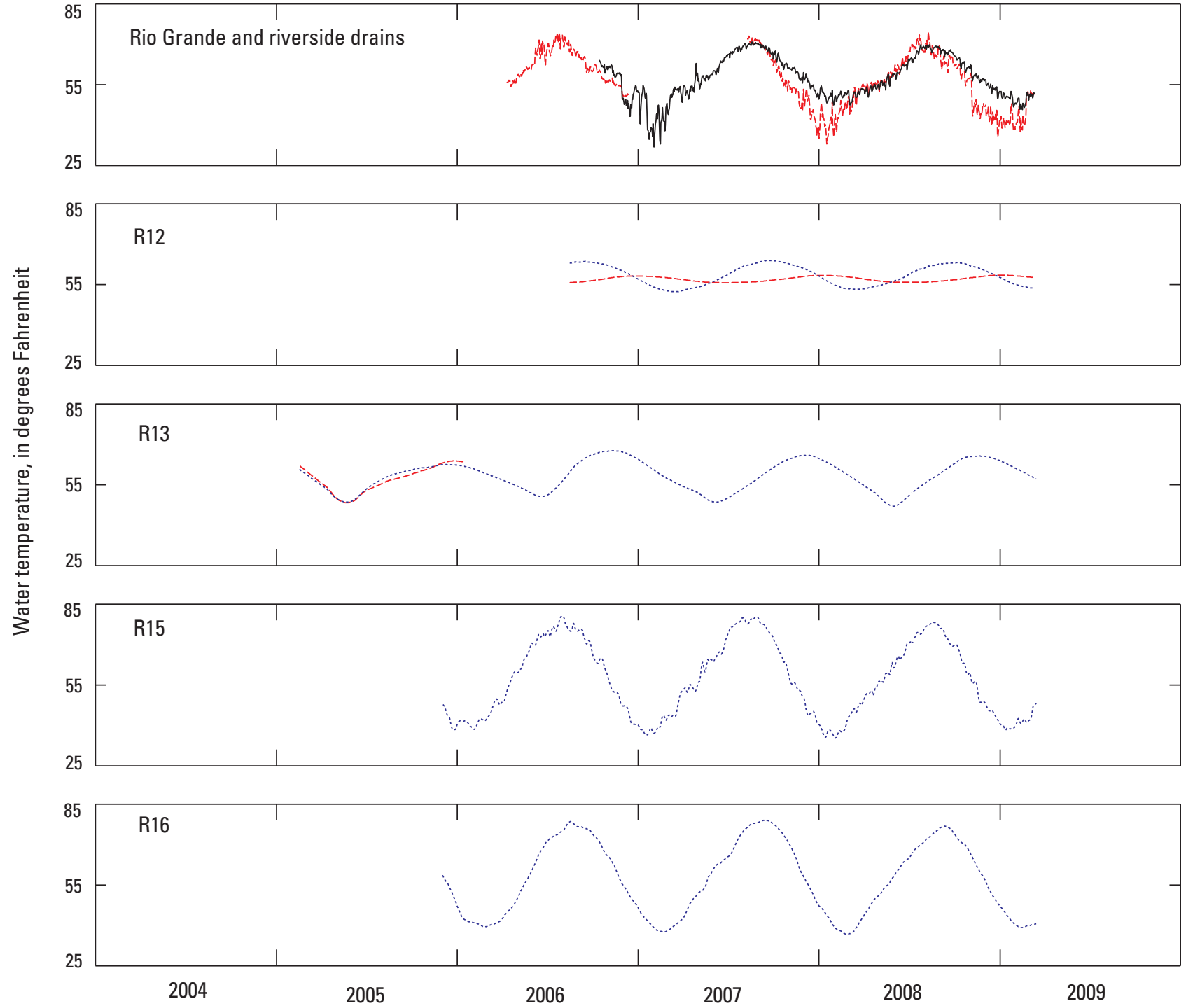

EXPLANATION

Surface water

Piezometer nests

......... Rio Grande

........ Piezometer a

East riverside drain

----- Piezometer b

West riverside drain

Figure 8h. Daily mean water temperature in the Rio Grande and riverside drains and daily mean groundwater temperature in piezometers at Rio Bravo transect 2, February 16, 2005-March 15, 2009. Piezometer locations are shown on figure 3d. 


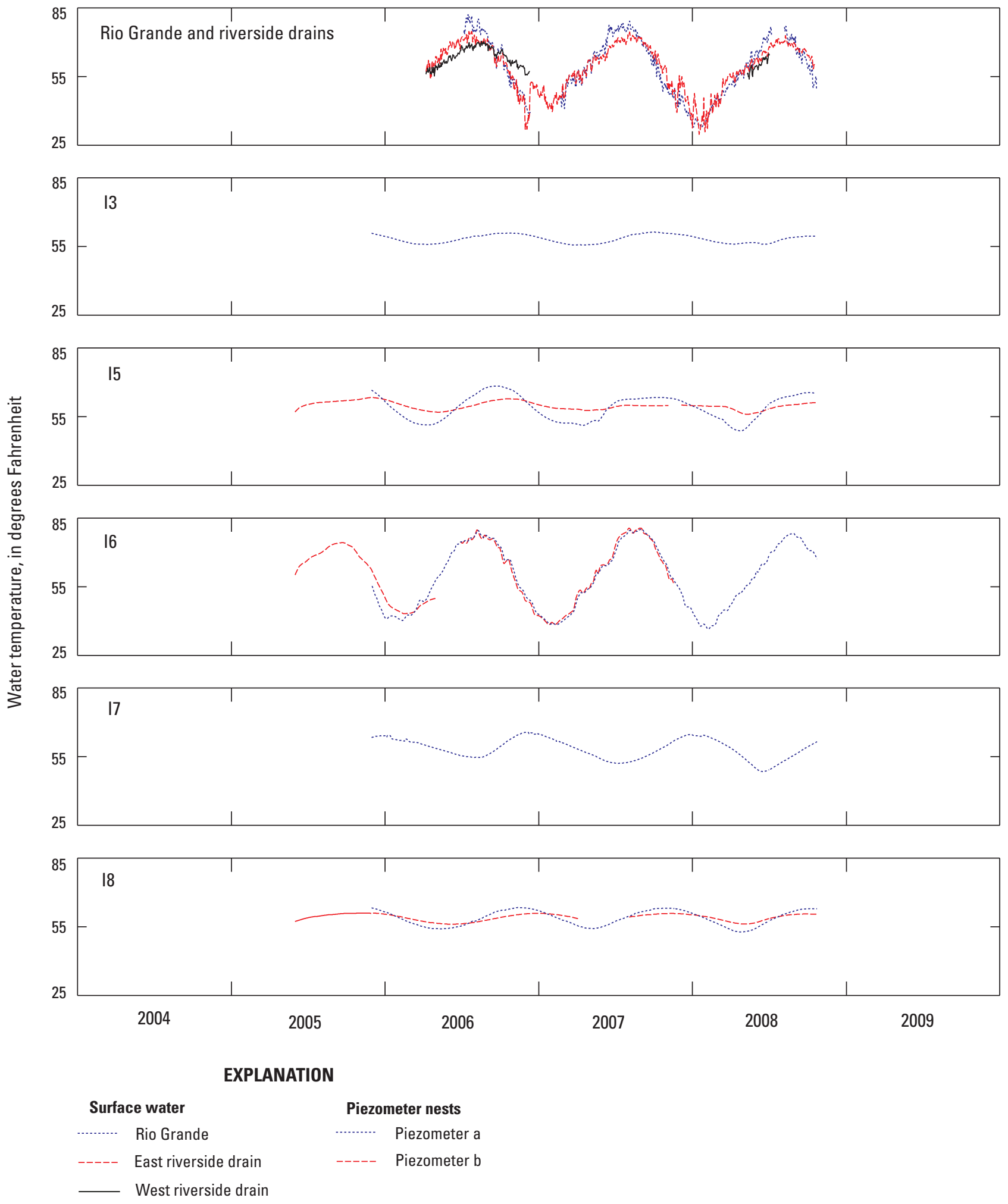

Figure 8i. Daily mean water temperature in the Rio Grande and riverside drains and daily mean groundwater temperature in piezometers at I-25 transect 1, April 22, 2005-October 24, 2008. Piezometer locations are shown on figure 3e. 

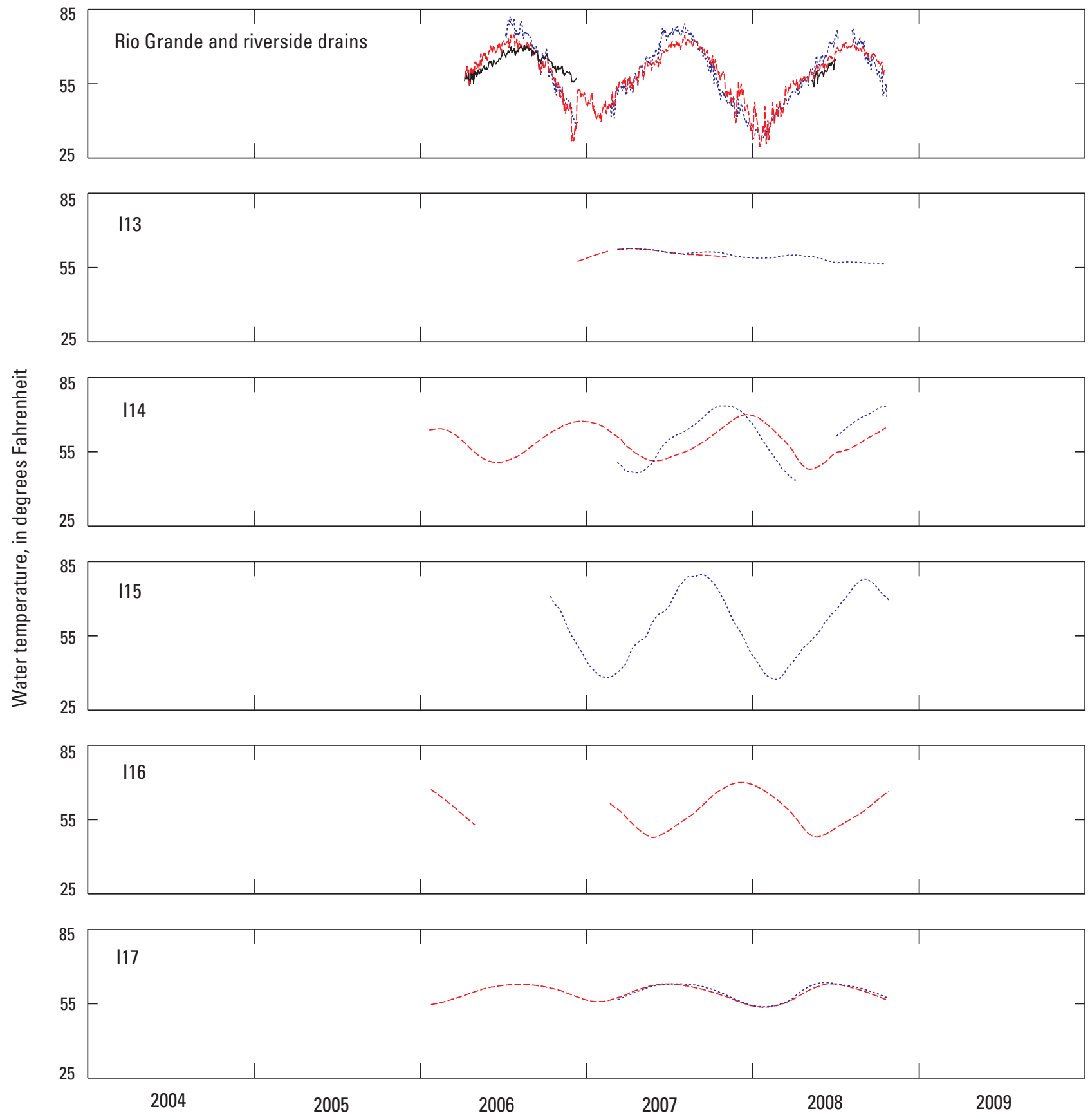

EXPLANATION

Surface water

Piezometer nests

Rio Grande

........- Piezometer a

East riverside drain

----- Piezometer b

West riverside drain

Figure 8j. Daily mean water temperature in the Rio Grande and riverside drains and daily mean groundwater temperature in piezometers at I-25 transect 2, January 21, 2006-October 27, 2008. Piezometer locations are shown on figure 3e. 
West Side of Rio Grande

Paseo del Norte
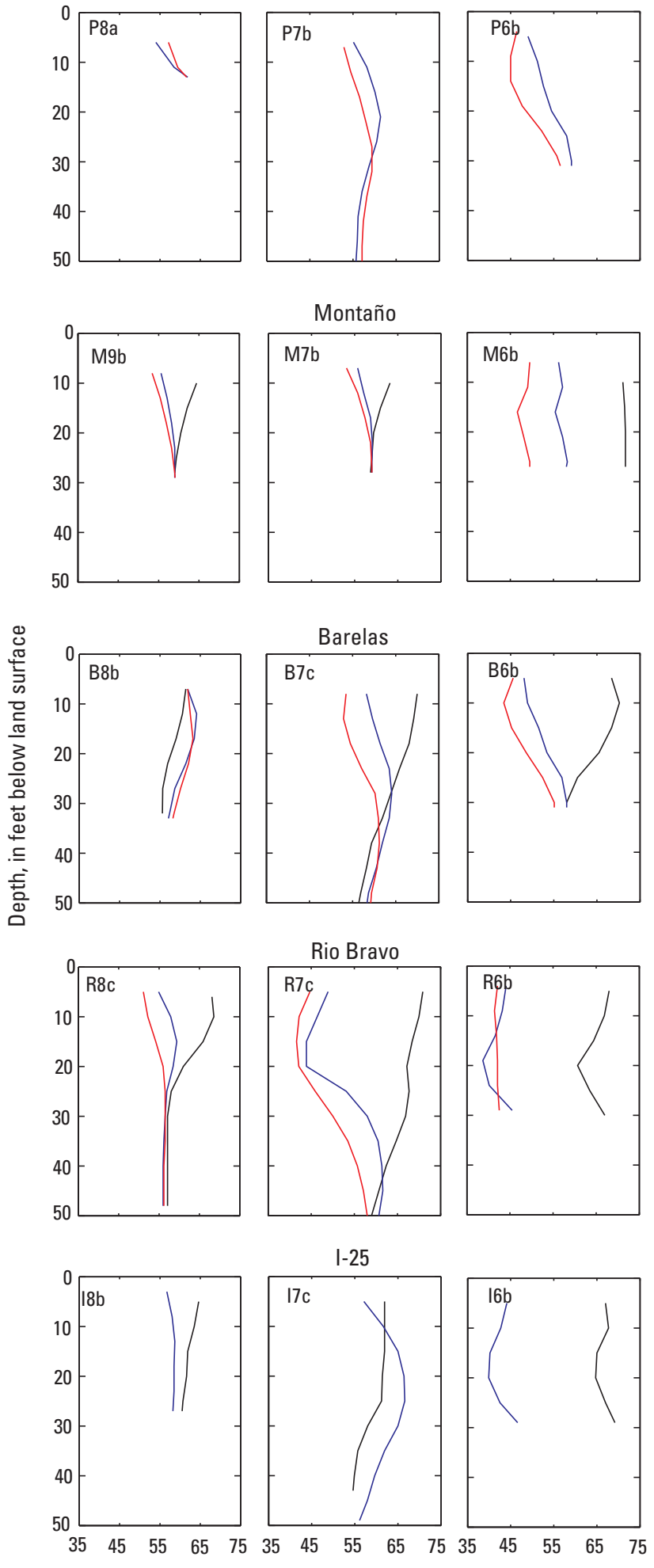

Temperature, in degrees Fahrenheit
East Side of Rio Grande

Paseo del Norte
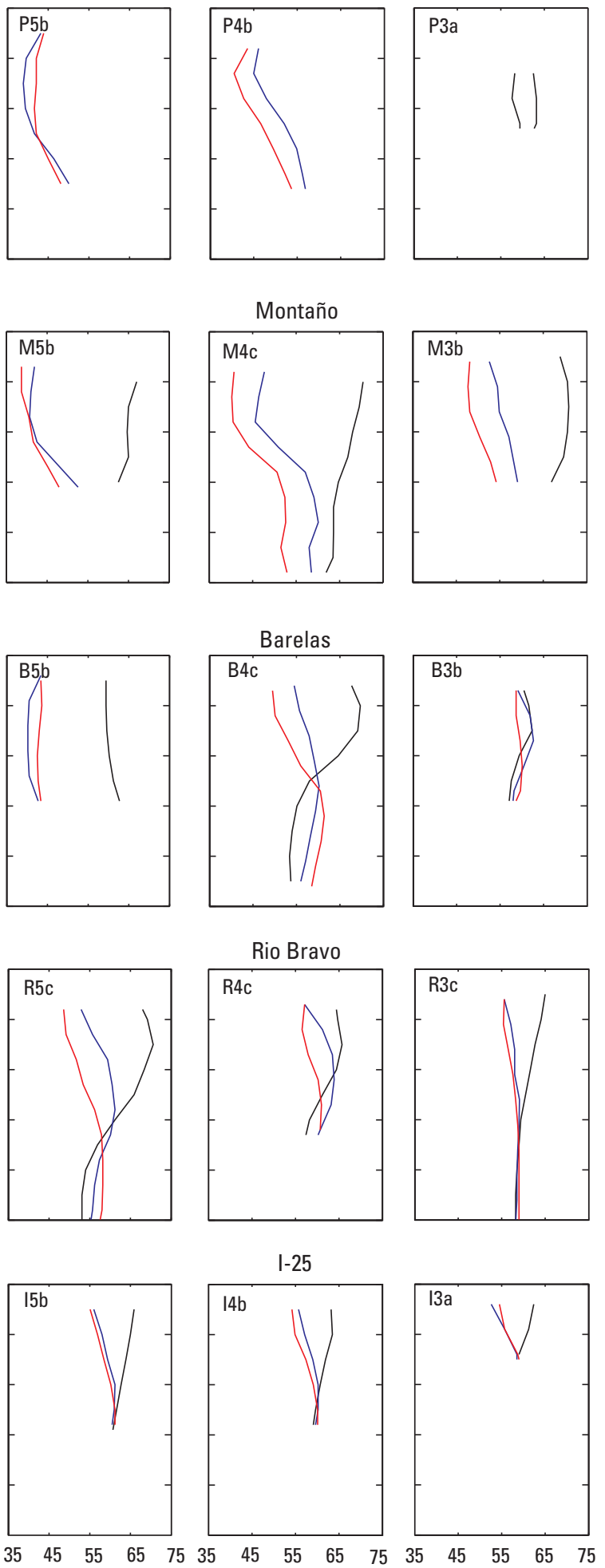

Temperature, in degrees Fahrenheit

\section{EXPLANATION}

October 2008 February 2009

Figure 9. Vertical temperature profiles in piezometers, winter nonirrigation season, October 2008, January 2009, and February 2009. 
Tulip-shaped temperature envelopes in figure 9, for example M3, M4, R5, and R7) show inflections between depths of 10 and $20 \mathrm{ft}$ that suggest heat fluxes from the Rio Grande are greatly reduced below about $30 \mathrm{ft}$. Similar results from Bartolino and Niswonger (1999) indicate that heat flux from the Rio Grande is limited below about $16 \mathrm{ft}$.

To distinguish between horizontal and vertical flow, Reiter (2001) presented temperature profiles in the Albuquerque area that alluded to the importance of cool horizontal flow; vertical flow alone could not cool water deeper in the aquifer to temperatures below those he observed at shallower depths. Reiter's (2001) conclusions can be applied to temperature profiles at piezometer nest I6, for example, where a negative temperature gradient to a depth of about $20 \mathrm{ft}$ is shown. At $20 \mathrm{ft}$ the temperature is $4^{\circ} \mathrm{F}$ to $5^{\circ} \mathrm{F}$ cooler than is observed at the shallower depths, indicating that cool horizontal flow is needed to reduce groundwater temperatures below that observed near the water table. Similarly, the importance of horizontal flux from the Rio Grande can be noted in temperature profiles from piezometer nests P4, P5, P6, P7, M3, M4, M5, B4, B6, B7, R4, R5, R6, R7, R8, I6, and I7 (fig. 9).

On the east side of the river, at the Montaño transects, temperature profiles indicate that the seasonal temperatureextinction depth is below the depth of observation. The shape of the temperature envelope at piezometer nest M4 indicates that heat from the Rio Grande is transported to depths greater than $50 \mathrm{ft}$ and that horizontal flux from the Rio Grande must be high to account for a range of $10^{\circ} \mathrm{F}$ at that depth. The curvature of the M4 profiles indicates that multiple zones of horizontal flow exist at depths of 10 to $20 \mathrm{ft}$ and $45 \mathrm{ft}$. These zones may be separated by less permeable material in the interval from 30 to $40 \mathrm{ft}$. The compressed nature of the temperature profiles at M7, M9, R3, R8, I3, I4, and I5 provide a sharp contrast to the variability in temperature profiles on the east side of the Rio Grande at Montaño. Compressed profiles generally indicate the presence of a discharge zone or low rates of groundwater flux (Reiter, 2001; Anderson, 2005).

\section{Conceptual Model}

The volume of water infiltrating from the Rio Grande that is intercepted by the riverside drains is dependent on the local hydraulic gradient, aquifer properties, and configuration of the groundwater-flow field. In the Albuquerque area, alluvial aquifer hydrology is complicated by a heterogeneous distribution of aquifer properties and anthropogenic influences on the system. Groundwater data collected at each transect in this study were used to evaluate geologic and anthropogenic influences and present a general understanding of groundwater flow in the alluvial aquifer.

A simple conceptual model of flow in figure 10 indicates that the groundwater table gently slopes from the Rio Grande towards riverside drains and the outer boundaries of the inner valley. Water infiltrating from the Rio Grande initially moves in a vertical direction below the bed of the river (Bartolino and Niswonger, 1999). As flow spreads farther into the alluvial aquifer, vertical gradients become small relative to horizontal gradients at depths less than $30 \mathrm{ft}$ and flow becomes primarily horizontal. The slope of the water-table surface may be strongly controlled by the riverside drains and, in a broader sense, influenced by other more distal hydrologic boundary conditions, such as groundwater withdrawals by wells. Riverside drains may induce localized vertical gradients that result in flow converging on the drain from all directions (West Riverside Drain in fig. 10). Alternatively, drains may intercept groundwater flow only from the shallowest part of the aquifer (East Riverside Drain in fig. 10).

Aquifer hydraulic conductivity also influences the slope of the water-table surface and rates of flux from the river. Deposits comprising the alluvial aquifer are a heterogeneous mix of sediments that overlie the more competent and coarser material of the Santa Fe Group aquifer system (Hawley and Haase, 1992). In general, descriptions of core from the alluvial aquifer indicate that the sediments of the alluvial aquifer grades from cobbles, gravels, sands, and silts to gravels, sands, silts, and clays in a downstream direction through the Albuquerque area. Clay layers can be hydrologically significant between the Barelas and Rio Bravo bridges (Bartolino and Sterling, 2000) where clay-layer thicknesses of $13 \mathrm{ft}$ have been observed (Roark, 2001). The influence of these local-scale heterogeneities is dependent on the continuity and extent of the deposit and its location within the flow system. Fine-grain deposits with low hydraulic conductivity can locally impede groundwater flow and reduce the rates of horizontal flow through the alluvial aquifer. Coarsergrain sands and gravels may allow higher rates of horizontal groundwater flow and infiltration from the river.

\section{Estimation of Horizontal Groundwater Flux from the Rio Grande}

Horizontal groundwater flux from the Rio Grande to the alluvial aquifer east and west of the river was estimated using results from calculations of horizontal hydraulic gradients (determined from analysis of hydraulic-head data), analysis of slug-test data, and heat-transport modeling. In the following report sections the portions of transect 1 and 2 that are east of river are referred to as the "east transects" and the portion of transects 1 and 2 that are west of the river are referred to as the "west transects".

\section{Horizontal Hydraulic Gradients}

Water-level data from shallow piezometers indicated that the median annual horizontal hydraulic gradients in the alluvial aquifer ranged from 0.011 (Barelas west) to 0.002 (I-25 east) (table 3). Generally, horizontal hydraulic gradients 


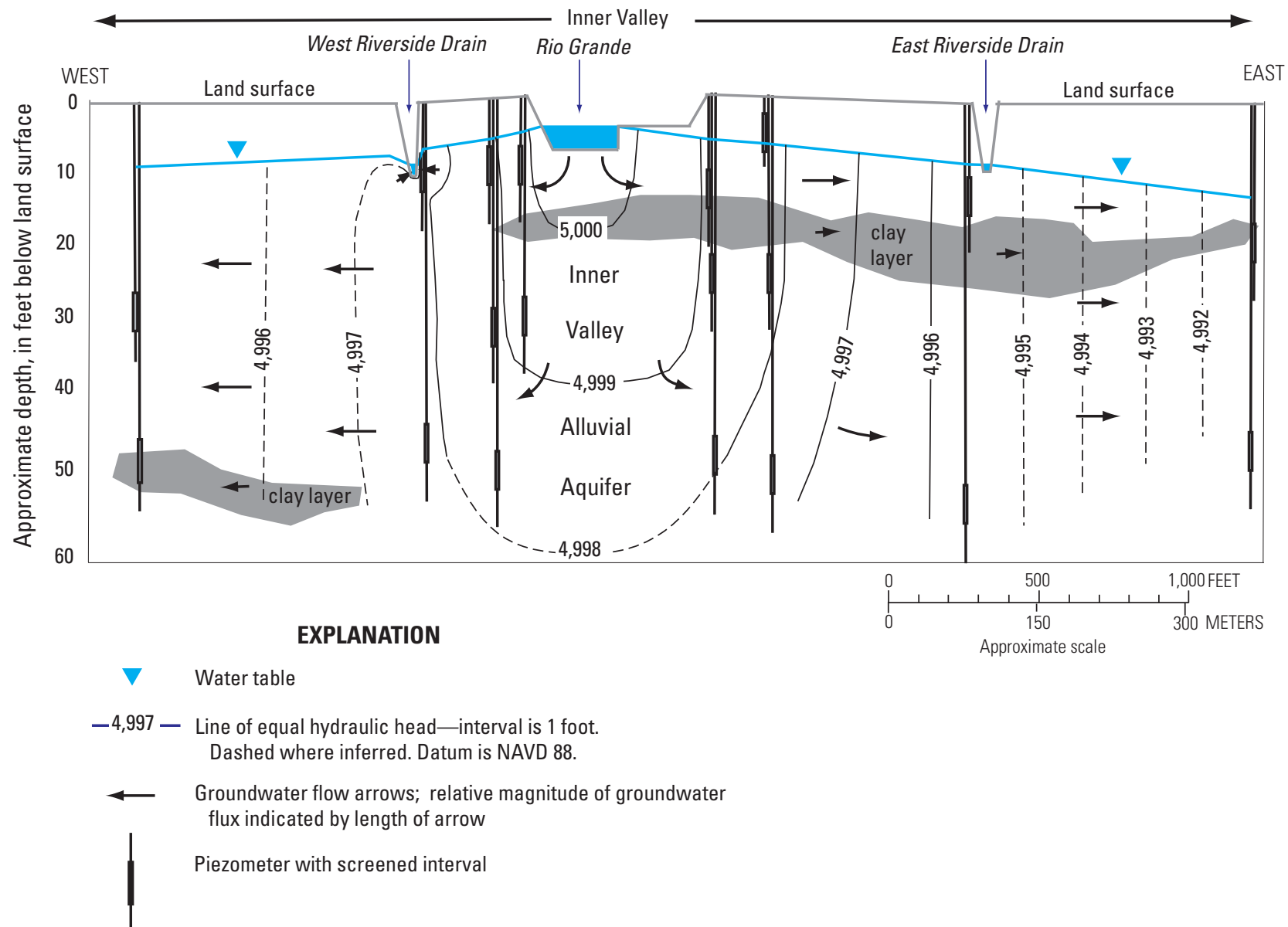

Figure 10. Conceptual model of groundwater flow from the Rio Grande through the Rio Grande inner valley alluvial aquifer.

increased from the Paseo del Norte to the Barelas transects, and decreased from the Barelas to the I-25 transects. Hydraulic gradients on the east and west sides of the Rio Grande differed by a factor of 1.6 or less at the Paseo Del Norte, Barelas, and Rio Bravo transects and differed by a factor of 2.5 or more at the Montaño and I-25 transects (table 3).

Relative to the downstream direction of the Rio Grande channel at each transect, the direction of groundwater flow on the east side of the river ranged from 71 to 89 degrees counterclockwise from the channel and on the west side ranged from 68 to 78 degrees clockwise from the channel (table 3). Between the Paseo Del Norte and Barelas bridges, the direction of flow relative to the river varied by less than 3 degrees, although noticeable differences in gradient directions were computed for the east and west sides of this reach of the Rio Grande (table 3). East of the Rio Grande between the Paseo Del Norte transects and the Barelas transects, flow was nearly perpendicular to the river. On both sides of the river, the direction of groundwater flow away from the river became less perpendicular in the downstream direction.

\section{Darcy Flux}

Darcy flux through the alluvial aquifer at each of the five transect locations was calculated using daily mean hydraulic gradients, which were calculated using shallow piezometer data and median hydraulic conductivities from slug-test results (this study) and hydraulic conductivities report by Tiedeman and others (1998) (fig. 11). The periods for which Darcy flux was calculated varied from transect to transect, depending on the availability of water-level data, from early 2006 through late 2008 (Barelas) to November-December 2008 (Paseo Del Norte) (fig. 11).

The Darcy fluxes shown in figure 11 represent the linear flux of groundwater flowing through a unit area (in this case one square foot) of aquifer material per day. Groundwater fluxes through the alluvial aquifer calculated by using median hydraulic conductivities from slug tests $\left(q_{\text {slug }}\right)$ (fig. 6) ranged from about $0.1 \mathrm{ft} / \mathrm{d}$ (Rio Bravo east and Montaño west) to about $0.7 \mathrm{ft} / \mathrm{d}$ (Barelas west) (fig. 11). The differences in $q_{\text {slug }}$ from one location to another appear to be a function of both hydraulic gradient (table 3 ) and hydraulic conductivity 

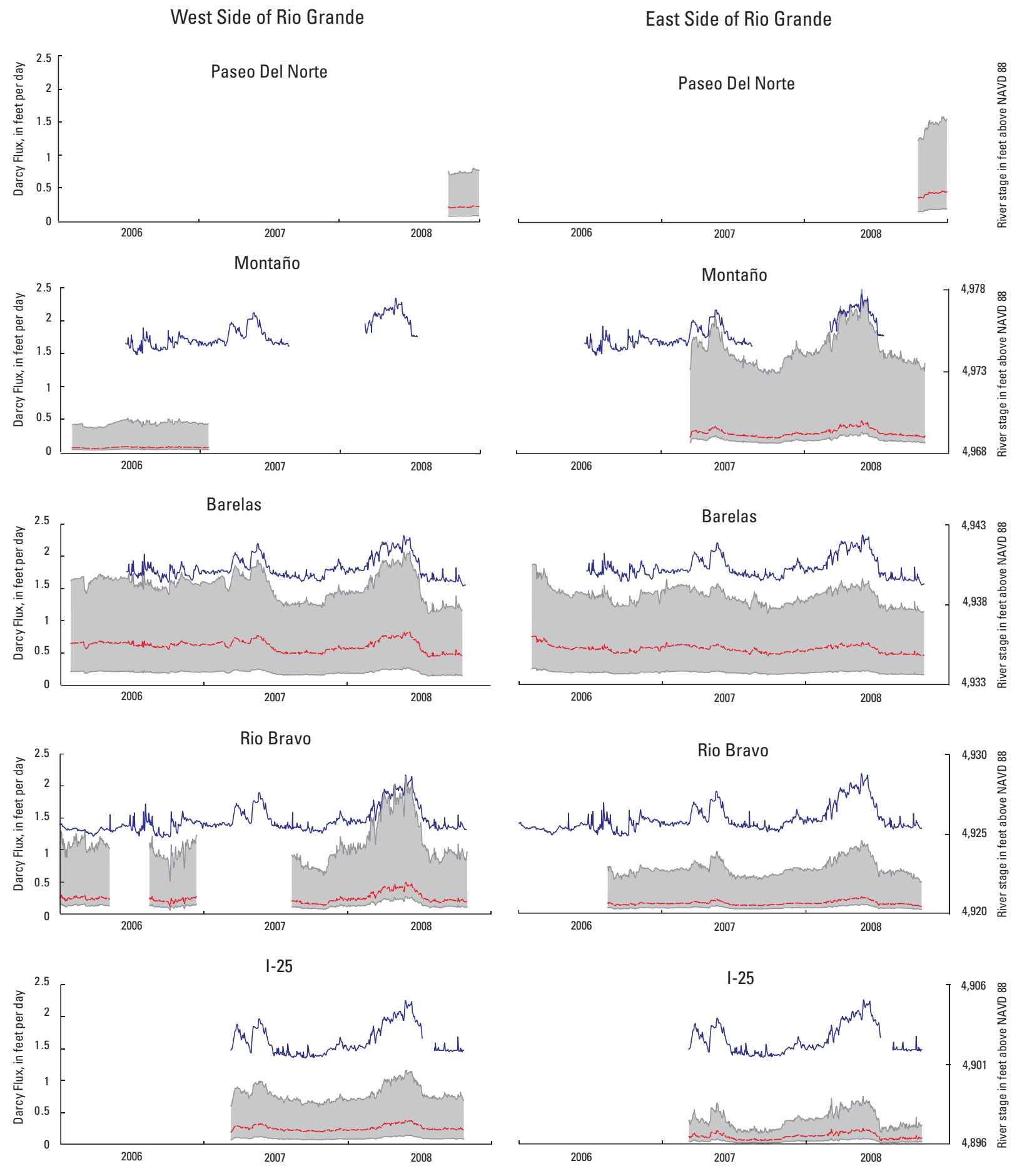

\section{EXPLANATION}

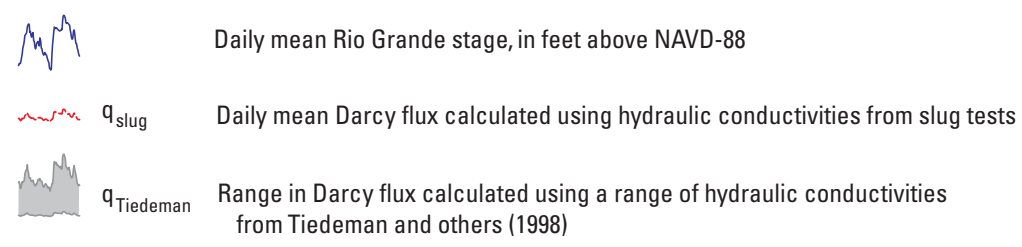

Figure 11. Daily mean Rio Grande stage and daily mean Darcy fluxes calculated from hydraulic gradients and hydraulic conductivities from slug tests, and from hydraulic gradients and a range of hydraulic conductivities (Tiedeman and others, 1998), Rio Grande inner valley alluvial aquifer, 2006-8 . 
(table 2). The $q_{\text {slug }}$ results indicate that the highest rates of groundwater flow through the alluvial aquifer occurred on the east and west sides of the river at the Barelas transects and correspond to the highest median hydraulic conductivity $(60 \mathrm{ft} / \mathrm{d})$ from slug test analysis (fig. 6) and high daily mean hydraulic gradients (table 3 ). The slowest rates of groundwater flow were found to correspond to transects with the lowest daily mean gradients (Montaño west and I-25 east).

With the exception of the Montaño and I-25 transects, the range of $q_{\text {slug }}$ values near each respective bridge location generally were similar on both sides of the river. The differing values of $q_{\text {slug }}$ at Montaño east and Montaño west were attributed to much lower gradients on the west side than on the east side of this location. Increases in $q_{\text {slug }}$ corresponded to periods of high flow in the Rio Grande at all locations. The Darcy flux $q_{\text {slug }}$ response to stage changes in the Rio Grande varied from 18 percent about the mean at Montaño east to 97 percent about the mean at Rio Bravo west. Although fluxes east and west of the river at the Barelas and Rio Bravo bridge locations were relatively uniform, hydraulic gradients on the west side of these transects were slightly more responsive to changes in river stage.

A range of fluxes $\left(q_{\text {tiedeman }}\right)$ through the alluvial aquifer was calculated by using a plausible range of hydraulic conductivities (20 to $150 \mathrm{ft} / \mathrm{d}$ ) compiled by Tiedeman and others (1998) and the daily mean hydraulic gradients calculated in this study (fig. 11). Daily mean $q_{\text {slug }}$ values (fig. 11) plot near the lower end of the $q_{\text {tiedeman }}$ range because the Darcy flux $q_{\text {slug }}$ was constrained by site-scale hydraulic conductivities ranging from 5 to $160 \mathrm{ft} / \mathrm{d}$. The difference between $q_{\text {slug }}$ values relative to the range of $q_{\text {tiedeman }}$ values was likely the result of differences in bulk aquifer properties measured at different scales. Inverse modeling, also at basin scale, by Tiedeman and others (1998), resulted in a slightly larger range of hydraulic conductivities ( 73 to $209 \mathrm{ft} / \mathrm{d}$ ). Simulated hydraulic gradients produced when Tiedeman and others (1998) used a hydraulic conductivity of $73 \mathrm{ft} / \mathrm{d}$ best match the hydraulic gradients calculated for this study. Other simulations at basin scale have used hydraulic conductivities of $24 \mathrm{ft} / \mathrm{d}$ (Sanford and others, 2003) and $45 \mathrm{ft} / \mathrm{d}$ (McAda and Barroll, 2002). The refinement of scale offered by the present study indicates that the magnitude of computed flux in the alluvial aquifer based solely on hydraulic-conductivity estimates from slug tests generally is consistent with that computed in large-scale models.

\section{Heat-Transport Modeling}

Results of calculations of horizontal groundwater flux in the alluvial aquifer using the Suzuki-Stallman method of heat-transport modeling are listed in table 4. Application of the Suzuki-Stallman method yields one set of $q_{a}$ and $q_{b}$ values for each temperature time-series pair analyzed. A temperature time-series pair consists of concurrent daily mean water-temperature data from two piezometers or from a surface-water gage and a piezometer. Because the water temperatures were observed to have a wavelength of about a year, $q_{a}$ and $q_{b}$ values are considered to represent mean annual flux. Some variance in the annual mean $q_{a}$ and $q_{b}$ values probably resulted from analyzing temperature data sets with differing time spans (table 4). Only one temperature timeseries pair was available for analysis at the Rio Bravo east and Rio Bravo west transects, and no data were available for the Paseo Del Norte west transects. Values of $a$ (the attenuation of the temperature wave) calculated from temperatures collected at $10 \mathrm{ft}$ and $20 \mathrm{ft}$ below land surface ranged from $2.0 \times 10^{-4}$ to $7.2 \times 10^{-3} \mathrm{ft}^{-1}$ and $1.3 \times 10^{-4}$ to $2.7 \times 10^{-2} \mathrm{ft}^{-1}$, respectively. Similarly, values of $b$ (the spatial frequency) calculated from daily mean temperatures collected at both the $10-\mathrm{ft}$ and $20-\mathrm{ft}$ depths ranged from $2.0 \times 10^{-2}$ to $1.0 \times 10^{-1} \mathrm{rad} / \mathrm{ft}$. All values of $a$ and $b$ were consistent for temperature time-series pairs that had multiple years of data.

Values of $a$ and $b$ were transformed to specific flux values of $q_{a}$ and $q_{b}$ by using type curves (fig. 5, table 4). At $10 \mathrm{ft}$, the specific flux values of $q_{a}$ and $q_{b}$, collectively, ranged from 0.09 to $0.74 \mathrm{ft} / \mathrm{d}$. At $20 \mathrm{ft}$, values of $q_{a}$ and $q_{b}$, collectively, ranged from 0.10 to $0.85 \mathrm{ft} / \mathrm{d}$. The average absolute deviation from the mean of all $q_{a}$ and $q_{b}$ values from 10 and $20 \mathrm{ft}$ was 0.12 , which indicates a relatively small range of $q_{a}$ and $q_{b}$ and lends confidence to the results in table 4 . Of some concern is the fact that the estimates of $q_{b}$ were systematically lower than the estimates of $q_{a}$. Moret (2007) suggests this may indicate that temperature signal phases were slightly distorted by the effects of temperature on water viscosity (cold water moves more slowly through the aquifer than warm water, so the timing of the arrival of temperature peaks and troughs at various locations in the aquifer would be slightly different than predicted by Stallman's (1965) equations).

The one-dimensional Suzuki-Stallman method assumes that groundwater flow in the alluvial aquifer was oriented orthogonal to the Rio Grande channel for all temperature time-series pairs used in this study. Gradient orientations (table 3), however, indicate groundwater flow was not orthogonal to the channel, so the assumption of orthogonality likely introduced some error into the analytical results for paired transects summarized in figure 12 . This error can be accounted for by adjusting the distance between measuring points in equations 12 and 13.

In addition, all of the temperature time-series pairs used in this study were spatially aliased. The values of $a$ and $b$ were determined by iteratively adding 1 -year increments to measured time lags until modeled sinusoidal curves matched observed data. In general, observed and modeled data fit well. Results of temperature time-series pair analyses were rejected if temperature time-series curves were not sinusoidal or if the values of $q_{a}$ and $q_{b}$ disagreed by more than 50 percent.

Values of $q_{a}$ and $q_{b}$ determined for each temperature timeseries pair were averaged to get a mean annual specific flux ( $q_{\text {heat }}$ in table 4). Box plots of $q_{\text {heat }}$ show the highest median value at Paseo Del Norte east $(0.52 \mathrm{ft} / \mathrm{d})$ and the lowest median value at I-25 east ( $0.23 \mathrm{ft} / \mathrm{d})$ (fig. 12). 
Table 4. Suzuki-Stallman results for horizontal flux between temperature time-series pairs in the Rio Grande inner valley alluvial aquifer, Albuquerque, New Mexico, 2006-8.

[Site locations shown in figures 3a-e; B, Barelas; I, I-25; M, Montaño; P, Paseo del Norte; R, Rio Bravo; a, shallow (10-foot depth) piezometer; b, mid-depth (20-foot depth) piezometer; $\mathrm{q}_{\mathrm{a}}$ and $\mathrm{q}_{\mathrm{b}}$, specific fluxes calculated from $\mathrm{a}$, temperature wave and $\mathrm{b}$, spatial frequency; $\mathrm{q}_{\text {heat }}$, average of $\mathrm{q}_{\mathrm{a}}$ and $\mathrm{q}_{\mathrm{b}}$; - , no data]

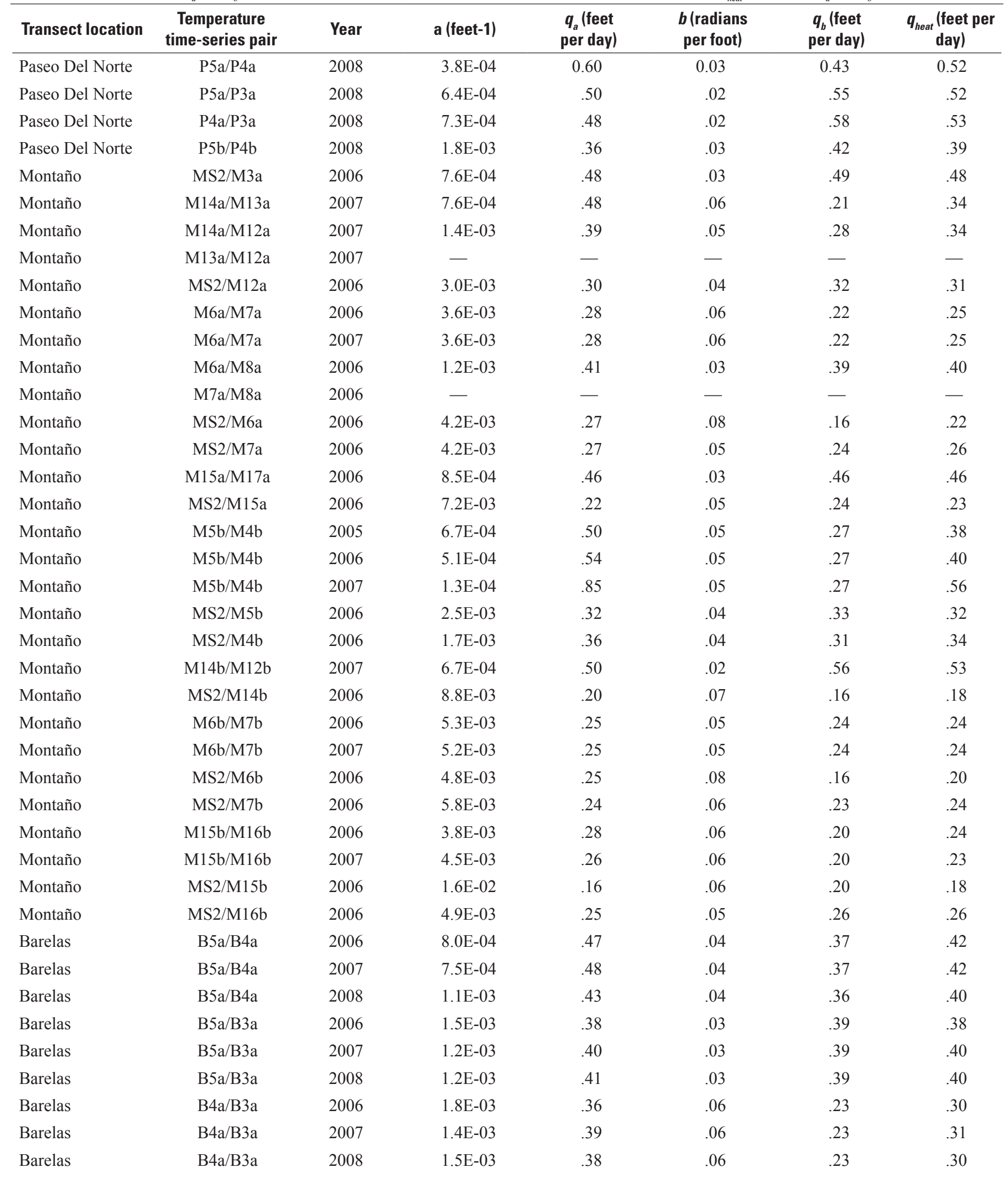


Table 4. Suzuki-Stallman results for horizontal flux between temperature time-series pairs in the Rio Grande inner valley alluvial aquifer, Albuquerque, New Mexico, 2006-8. - Continued

[Site locations shown in figures 3a-e; B, Barelas; I, I-25; M, Montaño; P, Paseo del Norte; R, Rio Bravo; a, shallow (10-foot depth) piezometer; b, mid-depth (20-foot depth) piezometer; $\mathrm{q}_{\mathrm{a}}$ and $\mathrm{q}_{\mathrm{b}}$, specific fluxes calculated from $\mathrm{a}$, temperature wave and $\mathrm{b}$, spatial frequency; $\mathrm{q}_{\text {heat }}$, average of $\mathrm{q}_{\mathrm{a}}$ and $\mathrm{q}_{\mathrm{b}}$; - no data]

\begin{tabular}{|c|c|c|c|c|c|c|c|}
\hline Transect location & $\begin{array}{l}\text { Temperature } \\
\text { time-series pair }\end{array}$ & Year & a (feet-1) & $\begin{array}{c}q_{a} \text { (feet } \\
\text { per day) }\end{array}$ & $\begin{array}{l}b \text { (radians } \\
\text { per foot) }\end{array}$ & $\begin{array}{c}q_{b} \text { (feet } \\
\text { per day) }\end{array}$ & $\begin{array}{c}q_{\text {heat }} \text { (feet per } \\
\text { day) }\end{array}$ \\
\hline Barelas & $\mathrm{BS} 2 / \mathrm{B} 5 \mathrm{a}$ & 2007 & $1.7 \mathrm{E}-03$ & .37 & .06 & .23 & .30 \\
\hline Barelas & $\mathrm{BS} 2 / \mathrm{B} 5 \mathrm{a}$ & 2008 & $1.8 \mathrm{E}-03$ & .36 & .06 & .23 & .30 \\
\hline Barelas & $\mathrm{BS} 2 / \mathrm{B} 4 \mathrm{a}$ & 2007 & $1.9 \mathrm{E}-03$ & .35 & .08 & .15 & .25 \\
\hline Barelas & $\mathrm{BS} 2 / \mathrm{B} 4 \mathrm{a}$ & 2008 & $2.8 \mathrm{E}-03$ & .31 & .08 & .15 & .23 \\
\hline Barelas & $\mathrm{BS} 2 / \mathrm{B} 3 \mathrm{a}$ & 2007 & $2.5 \mathrm{E}-03$ & .32 & .04 & .30 & .31 \\
\hline Barelas & B14a/B13a & 2006 & 7.1E-04 & .49 & .03 & .48 & .48 \\
\hline Barelas & $\mathrm{B} 14 \mathrm{a} / \mathrm{B} 13 \mathrm{a}$ & 2007 & $6.8 \mathrm{E}-04$ & .50 & .03 & .48 & .49 \\
\hline Barelas & $\mathrm{B} 14 \mathrm{a} / \mathrm{B} 12 \mathrm{a}$ & 2007 & $8.5 \mathrm{E}-04$ & .46 & .03 & .45 & .46 \\
\hline Barelas & $\mathrm{B} 13 \mathrm{a} / \mathrm{B} 12 \mathrm{a}$ & 2007 & $1.1 \mathrm{E}-03$ & .42 & .03 & .49 & .46 \\
\hline Barelas & $\mathrm{B} 6 \mathrm{a} / \mathrm{B} 7 \mathrm{a}$ & 2006 & 7.1E-04 & .49 & .03 & .45 & .47 \\
\hline Barelas & $\mathrm{B} 6 \mathrm{a} / \mathrm{B} 7 \mathrm{a}$ & 2007 & 7.1E-04 & .49 & .03 & .45 & .47 \\
\hline Barelas & $\mathrm{B} 6 \mathrm{a} / \mathrm{B} 7 \mathrm{a}$ & 2008 & $6.8 \mathrm{E}-04$ & .50 & .03 & .44 & .47 \\
\hline Barelas & BS2/B6a & 2007 & $6.1 \mathrm{E}-03$ & .23 & .09 & .13 & .18 \\
\hline Barelas & $\mathrm{BS} 2 / \mathrm{B} 6 \mathrm{a}$ & 2008 & $5.1 \mathrm{E}-03$ & .25 & .09 & .12 & .18 \\
\hline Barelas & $\mathrm{BS} 2 / \mathrm{B} 7 \mathrm{a}$ & 2007 & $1.7 \mathrm{E}-03$ & .37 & .05 & .24 & .30 \\
\hline Barelas & $\mathrm{BS} 2 / \mathrm{B} 7 \mathrm{a}$ & 2008 & $2.0 \mathrm{E}-03$ & .35 & .05 & .24 & .30 \\
\hline Barelas & $\mathrm{B} 15 \mathrm{a} / \mathrm{B} 16 \mathrm{a}$ & 2006 & $3.8 \mathrm{E}-04$ & .60 & .03 & .43 & .52 \\
\hline Barelas & $\mathrm{B} 15 \mathrm{a} / \mathrm{B} 16 \mathrm{a}$ & 2007 & $5.4 \mathrm{E}-04$ & .53 & .03 & .43 & .48 \\
\hline Barelas & $\mathrm{BS} 2 / \mathrm{B} 17 \mathrm{a}$ & 2007 & 7.3E-04 & .48 & .03 & .51 & .50 \\
\hline Barelas & $\mathrm{B} 4 \mathrm{~b} / \mathrm{B} 3 \mathrm{~b}$ & 2006 & $5.2 \mathrm{E}-03$ & .25 & .06 & .23 & .24 \\
\hline Barelas & $\mathrm{B} 4 \mathrm{~b} / \mathrm{B} 3 \mathrm{~b}$ & 2007 & 5.3E-03 & .25 & .06 & .23 & .24 \\
\hline Barelas & $\mathrm{B} 4 \mathrm{~b} / \mathrm{B} 3 \mathrm{~b}$ & 2008 & $5.6 \mathrm{E}-03$ & .24 & .06 & .22 & .23 \\
\hline Barelas & $\mathrm{BS} 2 / \mathrm{B} 4 \mathrm{~b}$ & 2007 & - & - & .10 & .10 & .10 \\
\hline Barelas & $\mathrm{BS} 2 / \mathrm{B} 4 \mathrm{~b}$ & 2008 & - & - & .10 & .10 & .10 \\
\hline Barelas & $\mathrm{BS} 2 / \mathrm{B} 3 \mathrm{~b}$ & 2007 & - & - & .08 & .15 & .15 \\
\hline Barelas & $\mathrm{BS} 2 / \mathrm{B} 3 \mathrm{~b}$ & 2008 & - & - & .08 & .15 & .15 \\
\hline Barelas & $\mathrm{B} 14 \mathrm{~b} / \mathrm{B} 13 \mathrm{~b}$ & 2006 & $8.1 \mathrm{E}-04$ & .47 & .03 & .46 & .46 \\
\hline Barelas & $\mathrm{B} 14 \mathrm{~b} / \mathrm{B} 13 \mathrm{~b}$ & 2007 & 7.9E-04 & .47 & .03 & .46 & .46 \\
\hline Barelas & $\mathrm{B} 14 \mathrm{~b} / \mathrm{B} 12 \mathrm{~b}$ & 2007 & 7.2E-04 & .49 & .03 & .50 & .50 \\
\hline Barelas & $\mathrm{B} 13 \mathrm{~b} / \mathrm{B} 12 \mathrm{~b}$ & 2007 & $1.8 \mathrm{E}-04$ & .77 & .02 & .57 & .67 \\
\hline Barelas & $\mathrm{BS} 2 / \mathrm{B} 14 \mathrm{~b}$ & 2007 & 2.7E-02 & .12 & .06 & .22 & .17 \\
\hline
\end{tabular}


Table 4. Suzuki-Stallman results for horizontal flux between temperature time-series pairs in the Rio Grande inner valley alluvial aquifer, Albuquerque, New Mexico, 2006-8.-Continued

[Site locations shown in figures 3a-e; B, Barelas; I, I-25; M, Montaño; P, Paseo del Norte; R, Rio Bravo; a, shallow (10-foot depth) piezometer; b, mid-depth (20-foot depth) piezometer; $\mathrm{q}_{a}$ and $\mathrm{q}_{b}$, specific fluxes calculated from $\mathrm{a}$, temperature wave and $\mathrm{b}$, spatial frequency; $\mathrm{q}_{\text {heat }}$, average of $\mathrm{q}_{\mathrm{a}}$ and $\mathrm{q}_{\mathrm{b}}$; - , no data]

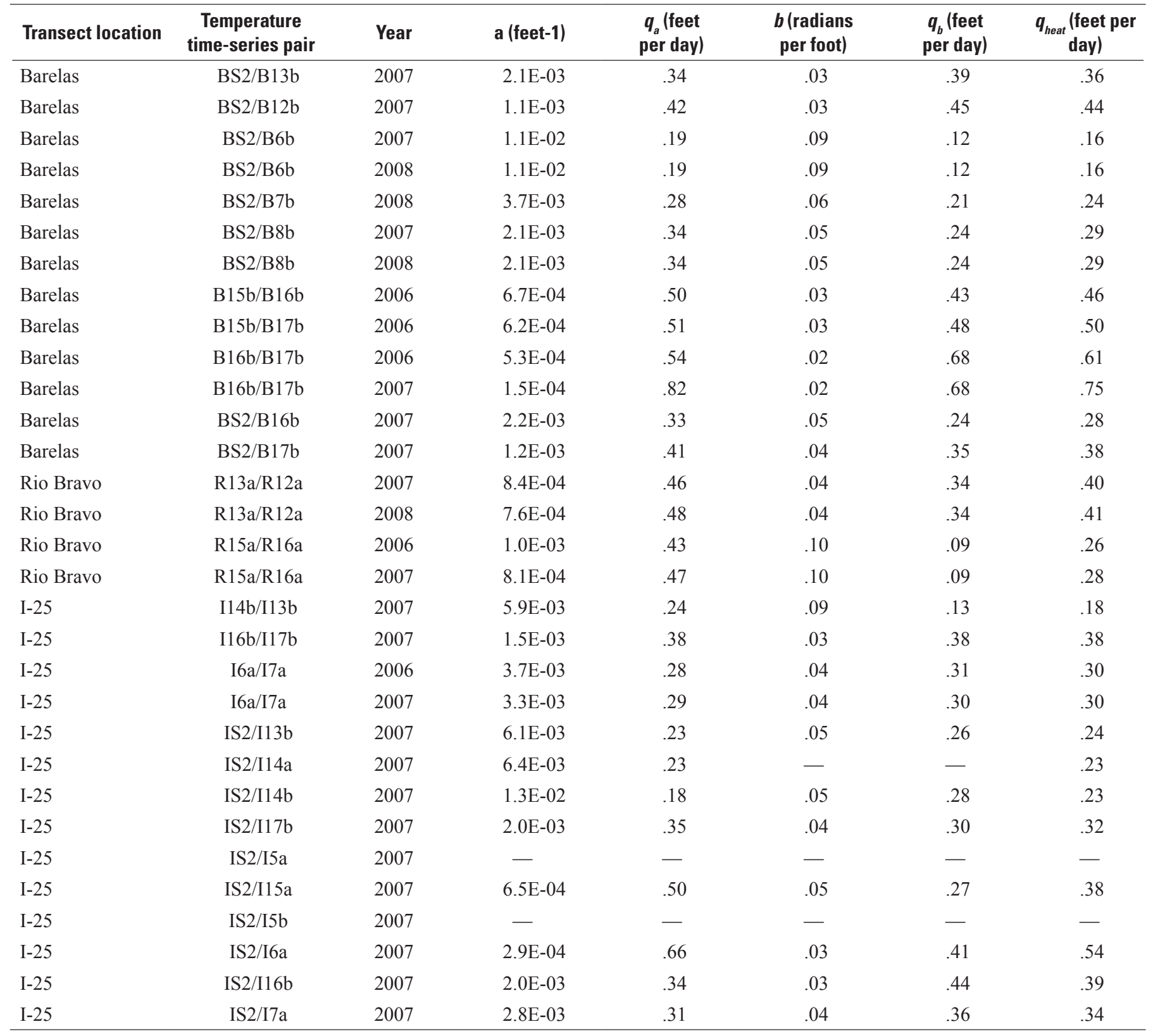




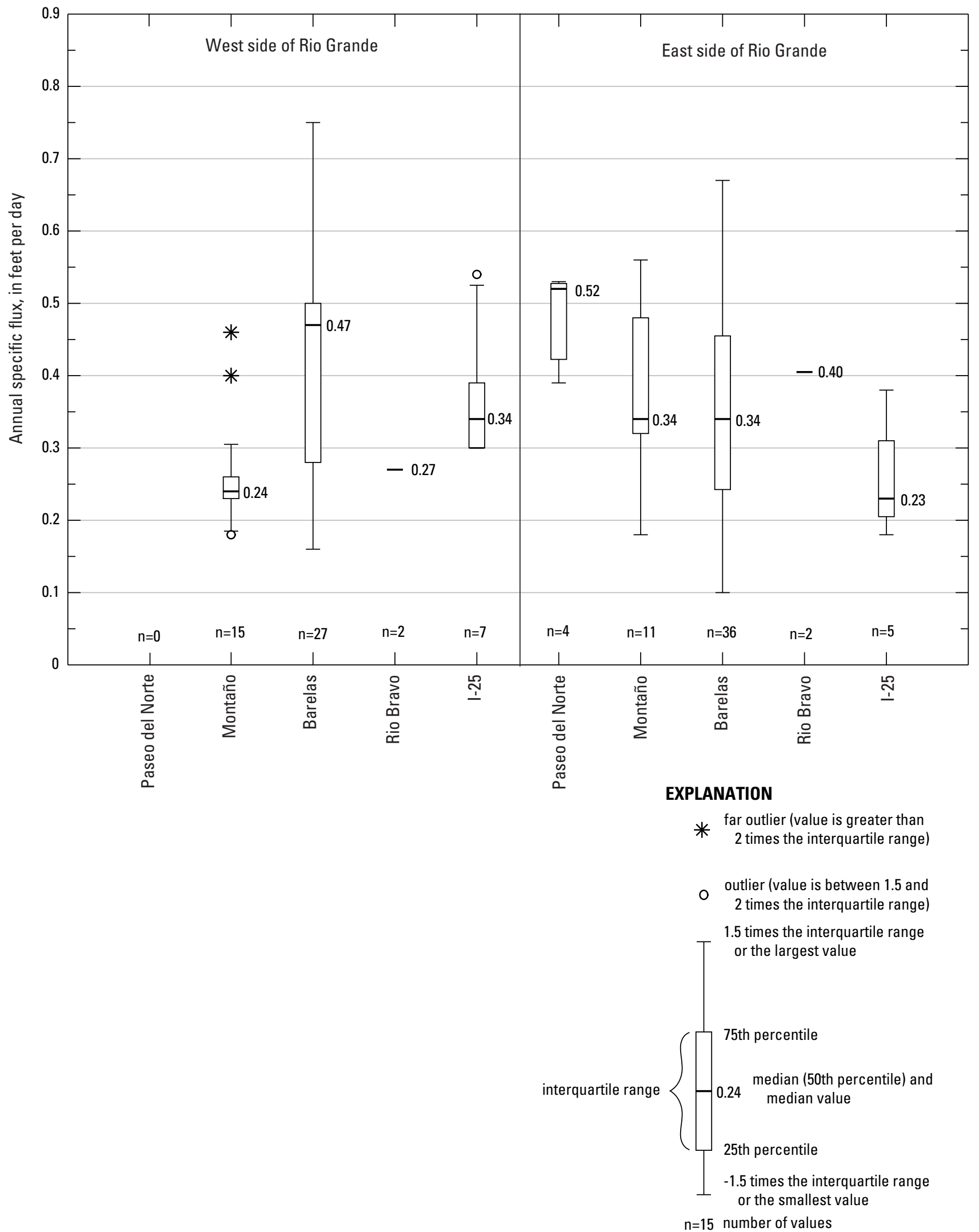

Figure 12. Summary of mean annual specific flux through the Rio Grande inner valley alluvial aquifer, Albuquerque, New Mexico, calculated using Suzuki-Stallman method. 
For all the east transects combined and all the west transects combined, the median of the mean annual $q_{\text {heat }}$ values from the east side of the river is higher $(0.35 \mathrm{ft} / \mathrm{d})$ than the median from the west side of the river $(0.32 \mathrm{ft} / \mathrm{d})$. Inspection of boxplots in figure 12 indicates that variance about the median increases with sample size. This is an indication that more temperature time-series pairs would have to be analyzed to fully characterize the range of specific flux at each set of transects. The relatively large sample sizes for both sides of the river at the Barelas transects (fig. 12) indicates that local scale geologic heterogeneities can result in a range of specific fluxes. The range of heterogeneities observed at the Barelas transects is likely present at all paired transect locations but not as well characterized by the smaller sample sizes at the other four transect locations.

\section{Model Comparison}

Median flux values computed from Darcy's law ranged from about 0.09 to $0.66 \mathrm{ft} / \mathrm{d}$ (table 5); annual median flux values computed from the Suzuki-Stallman method ranged from 0.23 to $0.52 \mathrm{ft} / \mathrm{d}$ (table 5). The two methods of computing flux agreed reasonably well, although in general, fluxes from the Suzuki-Stallman method were slightly higher than those computed through the use of Darcy's law (table 5). By using median annual gradients in table 3 and annual median specific fluxes computed by using the Suzuki-Stallman method, the horizontal hydraulic conductivity of the alluvial aquifer was estimated at each paired transect by using equation 4 and solving for hydraulic conductivity. Hydraulic-conductivity values from this analysis ranged from $30 \mathrm{ft} / \mathrm{d}$ (Montaño east) to $115 \mathrm{ft} / \mathrm{d}$ (I-25 east). The hydraulic conductivities calculated from the results of temperature analysis bracketed the median hydraulic-conductivity results (30 to $60 \mathrm{ft} / \mathrm{d}$ ) from the slug tests (fig. 6). The results computed using Darcy's law and results computed using the Suzuki-Stallman method (table 5) showed that groundwater fluxes through the alluvial aquifer generally are larger at and upstream of the Barelas transects than downstream.

\section{Riverside Drain Seepage Investigation}

To test the validity of the results from the Darcy's law and Suzuki-Stallman flux calculations, discharge was measured in riverside drains on both sides of the river north of the Montaño bridge (fig. 1) during a period of low flow on February 26, 2009 (table 6, fig. 7). All discharge measurements were conducted while wading, using a SonTek Flowtracker Handheld Acoustic Doppler Velocimeter (Rantz and others, 1982). On the Corrales Riverside Drain (west side of river), measurements were made at approximately $1-\mathrm{mi}$ intervals for about $5.6 \mathrm{mi}$ from below the head of the drain near Corrales to near the discharge point of the drain to the Rio Grande, south of the Montaño bridge. On the Albuquerque Riverside Drain (east side of river), one measurement was made near the Paseo del Norte bridge and another $3 \mathrm{mi}$ downstream near the Montaño bridge. The uncertainty in discharge measurements (Sauer and Meyer, 1992) ranged from 2.4 percent at east drain mile 3 to 4.8 percent at west drain mile 1 (table 6). Flow in the Rio Grande was not measured during this time; however, daily mean streamflow at the USGS Rio Grande at Alameda gaging station was $864 \mathrm{ft}^{3} / \mathrm{s}$ and at the Rio Grande at Albuquerque gaging station was $696 \mathrm{ft}^{3} / \mathrm{s}$ (see fig. 1 for locations). The daily mean streamflows would indicate that there was an average loss of water from the river to the aquifer of $168 \mathrm{ft}^{3} / \mathrm{s}$ on February 26, 2009, between the two gages. But, the error in the daily mean streamflow at these gages probably was on the order of 10 percent of the total flow or $\pm 86 \mathrm{ft}^{3} / \mathrm{s}$ at the Rio Grande at Alameda gaging station, and $\pm 70 \mathrm{ft}^{3} / \mathrm{s}$ at the Rio Grande at Albuquerque gaging station. The spans of possible error $\left(172 \mathrm{ft}^{3} / \mathrm{s}\right.$ at the Rio Grande at Alameda gaging station and $140 \mathrm{ft}^{3} / \mathrm{s}$ at the Rio Grande at Albuquerque gaging station) indicate that the difference in daily mean streamflow between the two gages $\left(168 \mathrm{ft}^{3} / \mathrm{s}\right)$ is within the range of, and could be attributable to, measurement error.

On the day that discharge was measured in the drains, the daily mean hydraulic gradients in the alluvial aquifer at the Paseo Del Norte west transects (0.005) was the same as the median annual hydraulic gradient calculated for the Paseo Del Norte west transects (table 3). Because river stage strongly influences hydraulic gradient and because river stage in February 2009 (figs. 7a and 7b) was relatively invariant, the hydraulic gradients at the Paseo Del Norte east and Montaño east and west transects presumably were similar to their respective median annual values (table 3 ).

Flow in the Corrales Riverside Drain increased by $1.4 \mathrm{ft}^{3} / \mathrm{s}$ from mile 2 to mile 4 (table 6 ) and decreased by $0.4 \mathrm{ft}^{3} / \mathrm{s}$ from mile 4 to mile 6 . Because there were no surfacewater inflows to the drain between miles 2 and 4 , the increased flow in the drain is attributable to seepage of groundwater from the aquifer to the drain. Groundwater discharging to the drain could have originated as seepage from the river to the aquifer or could have come from areas farther west of the drain. The increase in flow between drain miles 2 and 4 $(10,500 \mathrm{ft})$ represents seepage of groundwater to the drain at an average rate of about 12 cubic feet per day $\left(\mathrm{ft}^{3} / \mathrm{d}\right)$ per linear foot of drain.

The decrease in flow from mile 4 to 6 represents seepage of water from the drain to the aquifer. By design, a riverside drain slopes at a lower gradient than the river, thus ensuring that the drain terminus is higher than the river and that water in the drain can flow into the river. A consequence of this engineered design is that the water level in the drain for some distance upstream from the drain terminus is higher than the water level in the river and the groundwater table. The decrease in flow from mile 4 to 6 occurs near the downstream end of the Corrales Riverside Drain, where the drain design causes the water level in the drain to be higher than the groundwater table (compare water levels in fig. $3 \mathrm{~b}$ and fig. 7c for Corrales Riverside Drain gage MS3 and piezometer M9a). 
Table 50. Comparison of Darcy's law and Suzuki-Stallman calculations of horizontal groundwater flux in the Rio Grande inner valley alluvial aquifer, Albuquerque, New Mexico0.

[Site locations shown in figs0. 3a-e; ft, foot; rad, radians; q, Darcy flux; —, not reported0.]

\begin{tabular}{|c|c|c|}
\hline Transect Location & Darcy's law' median $q\left(q_{\text {slug }}\right.$, in feet per day) & Suzuki-Stallman median $\boldsymbol{q}\left(\boldsymbol{q}_{\text {heat }}\right.$, in feet per day) \\
\hline \multicolumn{3}{|c|}{ Paseo del Norte } \\
\hline West & 0.23 & - \\
\hline East & 0.36 & 0.52 \\
\hline \multicolumn{3}{|c|}{ Montaño } \\
\hline West & 0.09 & 0.24 \\
\hline East & 0.30 & 0.34 \\
\hline \multicolumn{3}{|c|}{ Barelas } \\
\hline West & 0.66 & 0.47 \\
\hline East & 0.54 & 0.34 \\
\hline \multicolumn{3}{|c|}{ Rio Bravo } \\
\hline West & 0.25 & 0.27 \\
\hline East & 0.18 & 0.40 \\
\hline \multicolumn{3}{|c|}{$1-25$} \\
\hline West & 0.25 & 0.34 \\
\hline East & 0.10 & 0.23 \\
\hline
\end{tabular}

${ }^{1}$ Calculated using median value of hydraulic conductivity from results of slug-test analyes (table 2)0.

Table 6. Seepage investigation discharge measurements in the Corrales and Albuquerque Riverside drains, Albuquerque, New Mexico, February 26, 2009.

[Site locations shown in fig. 1; DDMMSS.S, degrees minutes seconds]

\begin{tabular}{|c|c|c|c|c|c|c|}
\hline $\begin{array}{c}\text { Measurement location1 } \\
\text { (locations shown in figure 1) }\end{array}$ & $\begin{array}{l}\text { Latitude } \\
\text { (DDMMSS.S, } \\
\text { Datum is } \\
\text { NAD 83) }\end{array}$ & $\begin{array}{l}\text { Longitude } \\
\text { (DDMMSS.S, } \\
\text { Datum is } \\
\text { NAD 83) }\end{array}$ & $\begin{array}{l}\text { Measured } \\
\text { discharge } \\
\text { (cubic feet } \\
\text { per second) }\end{array}$ & $\begin{array}{c}\text { Measurement } \\
\text { uncertainty } \\
\text { (percent) }\end{array}$ & $\begin{array}{l}\text { Approximate } \\
\text { distance } \\
\text { from last } \\
\text { measurement } \\
\text { point (feet) }\end{array}$ & $\begin{array}{l}\text { Cumulative } \\
\text { distance along } \\
\text { drain (feet) }\end{array}$ \\
\hline Corrales Riverside Drain mile 1 & 351217.6 & -1063830.5 & 1.0 & 4.8 & 0 & 0 \\
\hline Corrales Riverside Drain mile 2 & 351115.0 & -1063908.8 & 1.6 & 2.9 & 7,400 & 7,400 \\
\hline Corrales Riverside Drain mile 4 & 350947.3 & -1064011.6 & 3.0 & 3.1 & 5,300 & 17,900 \\
\hline Corrales Riverside Drain mile 5 & 350855.0 & -1064054.0 & 2.7 & 2.8 & 6,500 & 24,400 \\
\hline Corrales Riverside Drain mile 6 & 350810.1 & -1064121.8 & 2.6 & 3.1 & 5,200 & 29,600 \\
\hline Albuquerque Riverside Drain mile 0 & 351055.2 & -1063852.9 & 58 & 3.0 & 0 & 0 \\
\hline Albuquerque Riverside Drain mile 3 & 350844.7 & -1064025.9 & 73 & 2.4 & 15,800 & 15,800 \\
\hline
\end{tabular}

${ }^{1}$ Mile marker location is approximate. More exact distances are shown in last two columns. 
Flow in the Albuquerque Riverside Drain increased by $15 \mathrm{ft}^{3} / \mathrm{s}$ between drain miles 0 and 3 . This increase in flow, which occurred over a distance of $15,800 \mathrm{ft}$, represents seepage of groundwater to the drain of at an average rate of about $82 \mathrm{ft}^{3} / \mathrm{d}$ per linear foot of drain. No surface-water inputs or overlapping drains were observed, so this increase in flow is attributable to seepage of groundwater from the aquifer to the drain.

Temperature profiles at the Montaño west transects (fig. 9) indicate that seasonal transport of heat from the Rio Grande during winter nonirrigation periods does not extend below 20-25 ft. The flux of water from the river to the aquifer was calculated to be $2.2 \mathrm{ft}^{3} / \mathrm{d}$ per linear foot of river by using (1) the median $q_{\text {slug }}$ at Montaño west of $0.09 \mathrm{ft} / \mathrm{d}$ (table 5), (2) a 1-foot width of aquifer measured parallel to the river, and (3) an assumption that all of the leakage from the Rio Grande is contained in the upper $25 \mathrm{ft}$ of the aquifer at the Montaño west transects. Under the same assumptions and by using the median $q_{\text {heat }}(0.24 \mathrm{ft} / \mathrm{d})$ calculated from the SuzukiStallman method for the Montaño west transects (table 5), the total flux was calculated to be $6.0 \mathrm{ft}^{3} / \mathrm{d}$ per linear foot of river. Assuming the Corrales Riverside Drain intercepted all of this flow, the $q_{\text {slug }}$ or $q_{\text {heat }}$ fluxes would only account for 18 to 50 percent, respectively, of the increase of flow in the drain (12 $\mathrm{ft}^{3} / \mathrm{d}$ per linear foot of drain) between miles 2 and 4 on February 26, 2009. Given a median annual hydraulic gradient of 0.003 at Montaño west (table 3), the hydraulic conductivity of the upper $25 \mathrm{ft}$ of alluvium would have to be $160 \mathrm{ft} / \mathrm{d}$ for discharge from the aquifer to the drain to account for 100 percent of the increase in flow in the riverside drain on February 26, 2009. As discussed previously, the design of the Corrales Riverside Drain at the Montaño west transects causes the water level in the drain to be higher than the groundwater table. So, the Darcy flux $q_{\text {slug }}$ and the Suzuki Stallman flux $q_{\text {heat }}$ probably are smaller than if they were measured in an area where the drain water level is lower than the groundwater table. Indeed, at the Paseo Del Norte west transects the median Darcy flux $q_{\text {slug }}$ is $0.23 \mathrm{ft} / \mathrm{d}\left(5.75 \mathrm{ft}^{3} / \mathrm{d}\right.$ per linear foot of river) and would account for about 48 percent of the increase in flow in the drain between miles 2 and 4 on February 26, 2009.

Temperature profiles at the Montaño east transects (fig. 9) indicate seasonal transport of heat from the Rio Grande during winter nonirrigation periods extends to depths greater than $50 \mathrm{ft}$. The flux of water from the river to the aquifer was calculated to be $15 \mathrm{ft}^{3} / \mathrm{d}$ per linear foot of river by using (1) the median $q_{\text {slug }}$ at the Montaño east transects of $0.30 \mathrm{ft} / \mathrm{d}$ (table 5), (2) a 1-foot width of aquifer measured parallel to the river, and (3) assuming that all of the leakage from the Rio Grande is contained in the upper $50 \mathrm{ft}$ of the aquifer at the Montaño east transects. Under the same assumptions and by using the mean flux calculated from the Suzuki-Stallman method for the Montaño east transects of $0.34 \mathrm{ft} / \mathrm{d}$ (table 5), the flux of water from the river to the aquifer was calculated to be $17 \mathrm{ft}^{3} / \mathrm{d}$ per linear foot of river. Assuming the Albuquerque Riverside Drain intercepted all this flow, the $q_{\text {sug }}$ or $q_{\text {heat }}$ fluxes would only account for 18 to 21 percent, respectively, of the increase in flow ( $82 \mathrm{ft}^{3} / \mathrm{d}$ per linear foot of drain) measured in the drain on February 26, 2009. Given an average annual hydraulic gradient of 0.010 at the Montaño east transects (table 3 ) the hydraulic conductivity of the upper $50 \mathrm{ft}$ of alluvium would have to be about $160 \mathrm{ft} / \mathrm{d}$ for discharge from the aquifer to the drain to account for 100 percent of the increase in flow in the riverside drain on February 26, 2009.

For all locations, flux rates for $q_{\text {slug }}$ ranged from $0.09 \mathrm{ft} / \mathrm{d}$ at the Montaño west transects to $0.66 \mathrm{ft} / \mathrm{d}$ at the Barelas west transects and flux rates for $q_{\text {heat }}$ ranged from $0.23 \mathrm{ft} / \mathrm{d}$ at the I-25 east transects to $0.52 \mathrm{ft} / \mathrm{d}$ at the Paseo del Norte east transects (table 5). Assuming that water leaking from the river to the aquifer is transmitted to the drains through a uniform $30 \mathrm{ft}$ aquifer thickness in the Albuquerque area, the $q_{\text {slug }}$ flux rates would range from 2.7 to $20 \mathrm{ft}^{3} / \mathrm{d}$ per linear foot of river and the $q_{\text {heat }}$ flux rates would range from 6.9 to $16 \mathrm{ft}^{3} / \mathrm{d}$ per linear foot of river.

At more local scales, Roark (2001) estimated river leakage to range from 0.47 to $2.1 \mathrm{ft}^{3} / \mathrm{d}$ per linear foot of river, Thorn (1995) indicated that leakage from the river averaged $74 \mathrm{ft}^{3} / \mathrm{d}$ per linear foot of river between the Paseo del Norte and Rio Bravo bridges, and Veenhuis (2002) indicated that leakage from the river averaged $123 \mathrm{ft}^{3} / \mathrm{d}$ per linear foot of river between the Bernalillo bridge and the Rio Bravo bridge. The comparison of these results with those of previous investigations suggests that calculated flux through the alluvial aquifer is strongly scale dependent and that the thickness of aquifer through which river water flows may be greater than indicated by the vertical temperature profiles. Depth of riverwater penetration into the aquifer could be further investigated using water chemistry to establish the boundary between water from the river and deeper aquifer water.

\section{Summary}

The Albuquerque area is the major population center in New Mexico and has two principal sources of water for municipal, domestic, commercial, and industrial uses in this area: groundwater from the Santa Fe Group aquifer system and surface water from the San Juan-Chama Diversion Project. Estimates indicated that from 1960 to 2002, groundwater withdrawals from the Santa Fe Group aquifer system have caused water levels to decline more than 120 feet in some places within the Albuquerque area. This has resulted in a great deal of interest in quantifying the river-aquifer interaction associated with the Rio Grande.

Previous researchers have used streambed permeameters, the transient response of the aquifer to a flood pulse, vertical profiles of temperature measurements, and calibrated 
numerical models to estimate the flux between the Rio Grande and the Santa Fe Group aquifer system. In contrast to previous more regional-scale studies, a study was designed to provide spatially detailed information about the amount of water that discharges from the Rio Grande to the adjacent aquifer in the Albuquerque area.

In 2003, the U.S. Geological Survey in cooperation with the Bureau of Reclamation, the Middle Rio Grande Endangered Species Collaborative Program, and the U.S. Army Corps of Engineers began a detailed characterization of the hydrogeology of the Rio Grande riparian corridor in the Albuquerque, New Mexico, area to provide hydrologic data and enhance the understanding of rates of water leakage from the Rio Grande to the alluvial aquifer, groundwater flow through the aquifer, and discharge of water from the aquifer to the riverside drains.

The study area extends about 18 miles along the Rio Grande in the Albuquerque area and the east and west edges of the study area are limited to areas within the inner valley adjacent to the Upper Corrales, Corrales, Albuquerque, and Atrisco Riverside Drains. The inner valley is approximately 2-3 miles wide and slopes about 5-6 feet per mile southward through the Albuquerque area. The riverside drains are ditches generally separated from the river by levees that are designed to intercept lateral groundwater flow from the river and prevent waterlogged-soil conditions east and west of the inner valley. Seepage to the riverside drains constitutes one of the main sources of groundwater discharge from the shallow alluvium.

The Rio Grande inner valley alluvial aquifer (alluvial aquifer) consists of coarse-grained axial channel deposits and post-Santa Fe Group sediments that underlie the presentday Rio Grande flood plain. The alluvium consists of unconsolidated to poorly consolidated, fine- to coarse-grain sand and rounded gravel with subordinate, discontinuous lens-shaped interbeds of fine-grain sand, silt, and clay. These deposits form an extensive shallow aquifer along the Rio Grande in the Albuquerque area and may be as much as 120 feet thick with an average thickness of 80 feet. The underlying Santa Fe Group aquifer system is composed primarily of gravel, sand, silt, and clay deposits that are approximately 14,000 feet thick in parts of the basin.

Piezometers and surface-water gages were installed in paired transects near five bridges in the Albuquerque area. Each transect included nested piezometers and surfacewater-stage gages configured in roughly straight lines and oriented perpendicular to the river and riverside drains. At each location, transects extended from the Rio Grande to just outside the riverside drains on both sides of the river and were spaced about 500 feet apart. The paired-transect configuration was chosen to facilitate definition of horizontal and vertical gradients at each location.

Continuous subsurface core samples were collected at each transect. Core samples were obtained to ensure that piezometer screens were placed in sand and gravel and to identify and locate any substantial changes in subsurface lithology that could potentially affect either horizontal or vertical groundwater movement. In total, 36 locations were cored within the study area at depths ranging from 25 to 55 feet.

Results from 35 slug tests performed in the alluvial aquifer for this study during January and February 2009 indicate that hydraulic-conductivity values ranged from 5 feet per day to 160 feet per day, with a median hydraulicconductivity for all transects of 40 feet per day. Slug-test results from piezometers on the east side of the river were not substantially different from those on the west side.

Hourly groundwater-level data were recorded from 164 piezometers screened at different intervals within the alluvial aquifer. Groundwater-level data were used to evaluate water-level trends, measure response to increases or decreases in river stage, and to calculate horizontal hydraulic gradients. Horizontal hydraulic gradients indicated that groundwater movement generally was away from the river and toward the drains.

Hourly groundwater temperatures were recorded at depths of 10 and 20 feet in selected piezometer nests. Large ranges in surface-water temperature were apparent: surfacewater temperatures ranged from $37^{\circ} \mathrm{F}$ in the winter to $79^{\circ} \mathrm{F}$ in the summer. Surface-water temperatures in the drains typically were similar to temperatures measured in the Rio Grande, but the magnitudes of fluctuations in the river were somewhat larger. Maximum and minimum water temperatures in piezometers generally indicate a decrease in amplitude and an increase in time lag of the temperature signal with increasing depth and distance from the river.

Vertical temperature profiles were collected during the months of October 2008 and January and February 2009 in the deepest piezometer at selected piezometer nests during the nonirrigation season. Temperature-profile data were collected to evaluate the depth of the alluvial aquifer that is influenced by leakage from the river. Temperatures were recorded at 5 -foot intervals from about 0.5 feet below the water surface to the bottom of each piezometer. Although piezometers were constructed with a screened interval of 5 feet near the bottom of the piezometer, the temperature of water in blank (nonscreened) casing was assumed to be the same as the temperature of water outside the casing. Groundwater temperatures recorded throughout the nonirrigation season were most variable at depths less than 30 feet and generally ranged from 40 to $70^{\circ} \mathrm{F}$.

A simple conceptual model of flow indicates that the groundwater table gently slopes from the Rio Grande towards riverside drains and the outer boundaries of the inner valley. Water infiltrating from the Rio Grande initially moves in a vertical direction below the bed of the river. As flow spreads farther into the alluvial aquifer, vertical gradients become small relative to horizontal gradients at depths less than 
30 feet and flow becomes primarily horizontal. The slope of the water-table surface may be strongly controlled by the riverside drains and, in a broader sense, influenced by other more distal hydrologic boundary conditions, such as groundwater withdrawals by wells. Riverside drains may induce localized vertical gradients that result in flow converging on the drain from all directions. Alternatively, drains may intercept groundwater flow only from the shallowest part of the aquifer.

Water-level data from shallow piezometers indicated that the median annual horizontal hydraulic gradients in the alluvial aquifer ranged from 0.011 to 0.002 . Generally, horizontal hydraulic gradients increase from the Paseo del Norte to the Barelas transects, and decrease from the Barelas to the I-25 transects. Hydraulic gradients on the east and west sides of the Rio Grande differed by a factor of 1.6 or less at the Paseo Del Norte, Barelas, and Rio Bravo transects and differed by a factor of 2.5 or more at the Montaño and I- 25 transects. Relative to the downstream direction of the Rio Grande channel at each transect, the direction of groundwater flow on the east side of the river ranged from 71 to 89 degrees counterclockwise from the channel and on the west side ranged from 68 to 78 degrees clockwise from the channel.

Groundwater fluxes through the alluvial aquifer calculated using median hydraulic conductivities from slug-tests $\left(q_{\text {slug }}\right)$ ranged from about 0.1 feet per day to about 0.7 feet per day. The differences in $q_{\text {slug }}$ from one location to another appear to be a function of both hydraulic gradient and hydraulic conductivity. The $q_{\text {slug }}$ results indicate that the highest rates of groundwater flow through the alluvial aquifer occurred on the east and west sides of the river at the Barelas transects and correspond to the highest median hydraulic conductivity from slug test analysis and high hydraulic gradients.

With the exception of the Montaño and I-25 transects, the range of $q_{\text {slug }}$ values near each respective bridge location generally were similar on both sides of the river. The differing values of $q_{\text {slug }}$ at Montaño east and Montaño west were attributed to much lower gradients on the west side than on the east side of this location. Although fluxes east and west of the river at the Barelas and Rio Bravo Bridge locations were relatively uniform, hydraulic gradients on the west side of these transects were slightly more responsive to changes in river stage. A range of fluxes $\left(q_{\text {tiedeman }}\right)$ through the alluvial aquifer was calculated by using a plausible range of hydraulic conductivities (20 to 150 feet per day) compiled from the literature and the daily mean hydraulic gradients calculated in this study. The difference between $q_{\text {slug }}$ values relative to the range of $q_{\text {tiedeman }}$ values was likely the result of differences in bulk aquifer properties measured at different scales. Results of calculations of horizontal groundwater flux $\left(q_{\text {heat }}\right)$ show that highest median value was at Paseo Del Norte east $(0.52$ feet per day) and the lowest median value at I- 25 east ( 0.23 feet per day). For all transects, the median of the mean annual $q_{\text {heat }}$ values from the east side of the river is higher than the median from the west side of the river. Flux values computed from Darcy's law and from the Suzuki-Stallman method agreed reasonably well, although in general, fluxes from the SuzukiStallman method were slightly higher than those computed through the use of Darcy's law.

To test the validity of the results from the Darcy's law and Suzuki-Stallman flux calculations, discharge was measured in riverside drains on both sides of the river north of the Montaño Bridge during a period of low flow on February 26, 2009. Flow in the Corrales Riverside Drain increased by 1.4 cubic feet per second from mile 2 to mile 4 and decreased by 0.4 cubic feet per second from mile 4 to mile 6 . The increase in flow between drain miles 2 and 4 represents seepage of groundwater to the drain of at an average rate of about 12 cubic feet per day per linear foot of drain. The decrease in flow from mile 4 to 6 represents seepage of water from the drain to the aquifer. Flow in the Albuquerque Riverside Drain increased by 15 cubic feet per second between drain miles 0 and 3 . This increase in flow represents seepage of groundwater to the drain at an average rate of about 82 cubic feet per day per linear foot of drain.

The flux of water from the river to the aquifer was calculated to be 2.2 cubic feet per day per linear foot of river by using the median $q_{\text {slug }}$ at Montaño west of 0.09 feet per day, a 1-foot width of aquifer measured parallel to the river, and an assumption that all of the leakage from the Rio Grande is contained in the upper 25 feet of the aquifer. Under the same assumptions and by using the mean $q_{\text {heat }}$ calculated from the Suzuki-Stallman method for the Montaño west transects of 0.24 feet per day, the total flux was calculated to be 6.0 cubic feet per day per linear foot of river. Assuming the Corrales Riverside Drain intercepted all of this flow, the $q_{\text {slug }}$ or $q_{\text {heat }}$ fluxes would only account for 18 to 50 percent, respectively, of the increase of flow in the drain ( 12 cubic feet per day per linear foot of drain) between miles 2 and 4 on February 26, 2009. Given a median annual hydraulic gradient of 0.003 at Montaño west, the hydraulic conductivity of the upper 25 feet of alluvium would have to be 160 feet per day for discharge from the aquifer to the drain to account for 100 percent of the increase in flow in the riverside drain on February 26, 2009.

The flux of water from the river to the aquifer was calculated to be 15 cubic feet per day per linear foot of river by using the median $q_{\text {slug }}$ at the Montaño east transects of 0.30 feet per day, a 1 -foot width of aquifer measured parallel to the river, and assuming that all of the leakage from the Rio Grande is contained in the upper 50 feet of the aquifer at the Montaño east transects. Under the same assumptions and by using the mean flux calculated from the SuzukiStallman method for the Montaño east transects of 0.34 feet per day, the flux of water from the river to the aquifer was calculated to be 17 cubic feet per day per linear foot of river. 
Assuming the Albuquerque Riverside Drain intercepted all this flow, the $q_{\text {slug }}$ or $q_{\text {heat }}$ fluxes would only account for 18 to 21 percent, respectively, of the increase in flow ( 82 cubic feet per day per linear foot of drain) measured in the drain on February 26, 2009. Given an average annual hydraulic gradient of 0.010 at the Montaño east transects the hydraulic conductivity of the upper 50 feet of alluvium would have to be about 160 feet per day for discharge from the aquifer to the drain to account for 100 percent of the increase in flow in the riverside drain on February 26, 2009. For all locations, assuming that water leaking from the river to the aquifer is transmitted to the drains through a uniform 30 feet aquifer thickness in the Albuquerque area, the $q_{\text {slug }}$ flux rates would range from 2.7 to 20 cubic feet per day per linear foot of river and the $q_{\text {heat }}$ flux rates would range from 6.9 to 16 cubic feet per day per linear foot of river.

The comparison of these results with those of previous investigations suggests that calculated flux through the alluvial aquifer is strongly scale dependent and that the thickness of aquifer through which river water flows may be greater than indicated by the vertical temperature profiles. Depth of riverwater penetration into the aquifer could be further investigated using water chemistry to establish the boundary between water from the river and deeper aquifer water.

\section{References}

Anderholm, S.K., and Bullard, T.F., 1987, Description of piezometer nests and water levels in the Rio Grande valley near Albuquerque, Bernalillo County, New Mexico: U.S. Geological Survey Open-File Report 87-122, 51 p.

Anderson, M.P., 2005, Heat as a ground water tracer: Ground Water, v. 43, no. 6, p. 951-968, November.

Barroll, Peggy, 2001, Documentation of the administrative groundwater model for the Middle Rio Grande Basin: Santa Fe, New Mexico Office of the State Engineer, Hydrology Bureau Report 99-3, 22 p.

Bartolino, J.R., 2003, The Rio Grande-Competing demands for a desert river, chap. 2 of Stonestrom, D.A., and Constantz, Jim, eds., Heat as a tool for studying the movement of ground water near streams: U.S. Geological Survey Circular 1260, p. 8-16.

Bartolino, J.R., and Cole, J.C., 2002, Ground-water resources of the Middle Rio Grande Basin, New Mexico: U.S. Geological Survey Circular 1222, 132 p.
Bartolino, J.R., and Niswonger, R.G., 1999, Numerical simulation of vertical ground-water flux of the Rio Grande from ground-water temperature profiles, central New Mexico: U.S. Geological Survey Water-Resources Investigations Report 99-4212, $34 \mathrm{p}$.

Bartolino, J.R., and Sterling, J.M., 2000, Electromagnetic surveys to detect clay-rich sediment in the Rio Grande inner valley, Albuquerque, New Mexico: U.S. Geological Survey Water-Resources Investigations Report 00-4003, 45 p.

Bexfield, L.M., and Anderholm, S.K., 2002, Estimated waterlevel declines in the Santa Fe Group aquifer system in the Albuquerque area, central New Mexico, predevelopment to 2002: U.S. Geological Survey Water-Resources Investigations Report 02-4233, $1 \mathrm{p}$.

Bexfield, L.M., and McAda, D.P., 2003, Simulated effects of ground-water management scenarios on the Santa Fe Group aquifer system, Middle Rio Grande Basin, New Mexico, 2001-40: U.S. Geological Survey Water-Resources Investigations Report 03-4040, $39 \mathrm{p}$.

Blasch, K.W., Constantz, Jim, and Stonestrom, David A., 2007, Thermal methods for investigating ground-water recharge, in Stonestrom, D.A., Constantz, Jim, Ferre, Ty P.A., and Leake, S.A., eds., Ground-water recharge in the arid and semiarid southwestern United States: U.S. Geological Survey Professional Paper 1703, Appendix 1, p. 351-373.

Bouwer, Herman, and Rice, R.C., 1976, A slug test for determining hydraulic conductivity of unconfined aquifers with completely or partially penetrating wells: Water Resources Research, v. 12, no. 3, p. 423-428.

Butler, J.J., 1998, The design, performance, and analysis of slug tests: Boca Raton, Fla., Lewis Publishers, 252 p.

Carslaw, H.S., and Jaeger, J.C., 1959, Conduction of heat in solids ( $2 \mathrm{~d}$ ed.): New York, Oxford University Press, $510 \mathrm{p}$.

Compton, R.R., 1962, Manual of field geology: New York, Wiley, 378 p.

Constantz, J.E., Niswonger, R.G., and Stewart, A.E., 2008, Analysis of temperature gradients to determine stream exchanges with ground water in Rosenberry, D.O., and LaBaugh, J.W., eds., Field techniques for estimating water fluxes between surface water and ground water: U.S. Geological Survey Techniques and Methods 4-D2, $128 \mathrm{p}$.

Constantz, Jim, Thomas, C.L., and Zellweger, G. W., 1994, Influence of diurnal variations in stream temperature on streamflow loss and groundwater recharge: Water Resources Research, v. 30, no. 12, p. 3253-3264. 
Constantz, Jim, Tyler, S.W., and Kwicklis, Edward, 2003, Temperature-profile methods for estimating percolation rates in arid environments: Vadose Zone Journal, v. 2, p. 12-24.

Connell, S.D., Love, D.W., and Dunbar, N.W., 2007, Geomorphology and stratigraphy of inset fluvial deposits along the Rio Grande Valley in the central Albuquerque Basin, New Mexico: New Mexico Geology, v. 29 , no. 1 , p. 31 .

Engdahl, N.B., Vogler, E.T, and Weissman, G.S., 2010, Evaluation of aquifer heterogeneity effects on river flow loss using a transition probability framework: Water Resources Research, v. 46, no. 1 [variously paged]. (Also available at http://dx.doi.org/10.1029/2009WR007903.)

Fetter, C.W., 1994, Applied hydrogeology (3d ed.): New York, Prentice-Hall, 690 p.

Freeman, L.A., Carpenter, M.C., Rosenberry, D.O., Rousseau, J.P., Unger, Randy, and McLean, J.S., 2004, Use of submersible pressure transducers in water-resources investigations: U.S. Geological Survey Techniques of Water-Resources Investigations 8-A3, 52 p.

Gould, Jaci, 1994, Middle Rio Grande channel permeameter investigations: Bureau of Reclamation Albuquerque Area Office, Middle Rio Grande Water Assessment Supporting Document, no. 11 [variously paged].

Hawley, J.W., and Haase, C.S., 1992, Hydrogeologic framework of the northern Albuquerque Basin: Socorro, N. Mex., New Mexico Bureau of Mines and Mineral Resources, Open-File Report 387, 176 p.

Hawley, J.W., and Whitworth, T.M.,1996, Hydrogeology and potential recharge areas for the basin and valleyfill aquifer systems and hydrogeochemical modeling of proposed artificial recharge of the Upper Santa Fe Aquifer, northern Albuquerque Basin, New Mexico: New Mexico Bureau of Mines and Mineral Resources, Open-File Report 402-D, 68 p.

Kernodle, J.M., McAda, D.P., and Thorn, C.R., 1995, Simulation of ground-water flow in the Albuquerque Basin, central New Mexico, 1901-1994, with projections to 2020: U.S. Geological Survey Water-Resources Investigations Report 94-4251, 114 p.

Kues, Georgianna, 1986, Ground-water levels and direction of ground-water flow in the central part of Bernalillo County, New Mexico, summer 1983: U.S. Geological Survey WaterResources Investigations Report 85-4325, 24 p.
McAda, D.P., 1996, Plan of study to quantify the hydrologic relations between the Rio Grande and the Santa Fe Group aquifer system near Albuquerque, central New Mexico: U.S. Geological Survey Water-Resources Investigations Report 96-4006, 58 p.

McAda, D.P., 2001, Simulation of a long-term aquifer test conducted near the Rio Grande, Albuquerque, New Mexico: U.S. Geological Survey Water-Resources Investigations Report 99-4260, 58 p.

McAda, D.P., and Barroll, Peggy, 2002, Simulation of ground-water flow in the middle Rio Grande Basin between Cochiti and San Acacia, New Mexico: U.S. Geological Survey Water-Resources Investigations Report 02-4200, 81 p.

Moret, G.J.M., 2007, Annual variations in groundwater temperature as a tracer of river-aquifer interactions: State College, Pennsylvania State University, Ph.D. dissertation, $153 \mathrm{p}$.

Myers, N.C., Finnegan, P.J., and Breedlove, J.D., 1999, Analysis of water-level data and ground-water flow modeling at Fort Riley, Kansas: U.S. Geological Survey Water-Resources Investigations Report 99-4115, 6 p.

National Climatic Data Center, 2011, Quality controlled local climatological data: National Oceanic and Atmospheric Administration, accessed December 1, 2011, at http://cdo.ncdc.noaa.gov/qclcd/QCLCD?prior=N.

Peter, K.D., 1987, Ground-water flow and shallow-aquifer properties in the Rio Grande inner valley south of Albuquerque, Bernalillo County, New Mexico: U.S. Geological Survey Water-Resources Investigations Report 87-4015, 29 p.

Rantz, S.E., and others, 1982, Measurement and computation of streamflow: U.S. Geological Survey Water-Supply Paper 2175, 2 v., 631 p.

Reiter, Marshall, 2001, Using precision temperature logs to estimate horizontal and vertical groundwater flow components: Water Resources Research, vol. 37, no. 3, p. 663-674.

Roark, D.M., 2001, Estimation of hydraulic characteristics in the Santa Fe Group aquifer system using computer simulations of river and drain pulses in the Rio Bravo study area, near Albuquerque, New Mexico: U.S. Geological Survey Water-Resources Investigations Report 01-4069, 52 p. 
Sanford, W.E., Plummer, L.N., McAda, D.P., Bexfield, L.M., and Anderholm, S.K., 2003, Use of environmental tracers to estimate parameters for a predevelopment-ground-waterflow model of the Middle Rio Grande Basin, New Mexico: U.S. Geological Survey Water-Resources Investigations Report 03-4286, 102 p.

Sauer, V.B., and Meyer, R.W., 1992, Determination of error in individual discharge measurements: U.S. Geological survey Open-File Report 92-144, 21 p.

Silliman, S.E., and Booth, D.F., 1993, Analysis of time-series measurements of sediment temperature for identification of gaining vs. losing portions of Juday Creek, Indiana: Journal of Hydrology, v. 146, p. 131-148.

Smerdon, J.E., Pollack, H.N., Cermak, Vladimir, Enz, J.W., Kresl, Milan, Safanda, Jan, and Wehmiller, J.F., 2004, Air-ground temperature coupling and subsurface propagation of annual temperature signals: Journal of Geophysical Research, v. 109, no. 21, D21107, 10 p.

Stallman, R.W., 1965, Steady one-dimensional fluid flow in a semi-infinite porous medium with sinusoidal surface temperature: Journal of Geophysical Research, v. 70, no. 12 , p. $2,821-2,827$.

Suzuki, Seitaro, 1960, Percolation measurements based on heat flow through soil with special reference to paddy fields: Journal of Geophysical Research, v. 65, no. 9, p. 2883-2885.

Thorn, C.R., 1995, Surface-water discharge and evapotranspiration rates for grass and bare soil along a reach of the Rio Grande, Albuquerque, New Mexico, 198995: U.S. Geological Survey Open-File Report 95-419, 23 p.

Tiedeman, C.R., Kernodle, J.M., and McAda, D.P., 1998, Application of nonlinear-regression methods to a groundwater flow model of the Albuquerque Basin, New Mexico: U.S. Geological Survey Water-Resources Investigations Report 98-4172, $90 \mathrm{p}$.

U.S. Census Bureau, 2003, Census of population and housing, New Mexico 2000-Summary population and housing characteristics: PHC-1-33, $114 \mathrm{p}$.

Veenhuis, J.E., 2002, Summary of loss between selected cross sections on the Rio Grande in and near Albuquerque, New Mexico: U.S. Geological Survey Water-Resources Investigations Report 02-4131, $30 \mathrm{p}$.

Vennard, J.K., and Street, R.L., 1982, Elementary fluid mechanics (6th ed.): New York, Wiley, 689 p. 


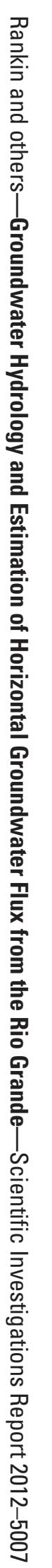

